\title{
OpERATIONAL Risk MANAGEMENT - IMPLEMENTING A BAYESIAN NETWORK FOR Foreign ExChange ANd Money Market SETTLEMENT
}

\author{
Dissertation \\ Presented for the Degree of Doctor of Philosophy at the Faculty of Economics \\ and Business Administration of \\ the University of Göttingen \\ by \\ Kwabena Adusei-Poku \\ from Kumasi, Ghana. \\ Göttingen, 2005
}


First Examiner: $\quad$ Prof. Dr. Walter Zucchini

Second Examiner: Prof. Dr. Wolfgang Benner

Date of oral exams: 26.08.2005 
To Edith,

for all the sacrifices you made. 



\section{Acknowledgements}

This work was completed during my stay at the Institute of Statistics and Econometrics of the Faculty of Economics and Business Administration, University of Goettingen while participating in the Ph.D program of the Center of Statistics. Like all major endeavours, one receives help, guidance and encouragement, which must be acknowledged.

I wish to thank my advisor Prof. Dr. Walter Zucchini for the guidance and encouragement he gave me throughout my stay at the Institute. I particularly appreciate his patience and willingness to help at all times without which this work would not have been completed. Many thanks to Marcelo G. Cruz of RiskMaths Consulting and Jack L. King of Genoa (UK) Limited for the comments and suggestions they provided during the initial stages of the work. Gerrit van den Brink and Helmut Heiming of Dresdner Bank deserve special thanks for helping with the supervision of the work and providing the needed practical insights as well as helping me appreciate operational risk the more. Particular thanks are also due to Patricia Licht and Edgar Peters.

Special thanks to Oleg Nenadic of the Institute of Statistics and Econometrics for the numerous help he provided in statistical programing and LaTex. Thanks to Prof. Dr. Wolfgang Benner and PD. Dr. Andreas Nickel for accepting to be my second and third examiners respectively. I also wish to thank Prof. Manfred Denker of the Center for Statistics for arranging the funding for this work.

Finally and most importantly, my most sincerest thanks to my wife, Edith, for her love, support and understanding, which I have greatly benefited from. Thank you Edith for proof reading the script and especially, caring for our children (Serwa and Kwame) during this major endeavour. 



\section{Contents}

1 Introduction and Motivation $\quad 1$

1.1 Introduction . . . . . . . . . . . . . . . . . . . . . 1

1.2 Motivation and Problem Description . . . . . . . . . . . . 2

1.3 Objectives of Research . . . . . . . . . . . . . . . . 3

1.4 Outline of the Thesis . . . . . . . . . . . . . 3

2 Operational Risk, Foreign Exchange and Money Market 5

2.1 Basel II, Operational Risk and Sarbanes-Oxley . . . . . . . . . . . 5

2.2 The Foreign Exchange and Money Markets . . . . . . . . . . 8

2.3 Operational Risk in Foreign Exchange and Money Market . . . . 10

2.4 Review of Operational Risk Methods . . . . . . . . . . . . . . . . 13

2.4.1 Models for Quantification and Capital Allocation . . . . . 13

2.4.1.1 Loss Distribution Approach . . . . . . . . . . 14

2.4.1.2 The Scorecard or Risk Drivers and Controls Approach ................. 17

2.4.1.3 Scenario-Based Approach _......... . 18

2.4.2 Models for Managing OR . . . . . . . . . . . . 19

2.5 Assessment of Bayesian Networks for Operational Risk Management 22

2.5.1 Prospects of Bayesian Networks for Operational Risk . . . 22

2.5.2 Criticisms of Bayesian Networks in Operational Risks Application ...................... 23

3 Bayesian Networks $\quad 25$ 
3.1 Introduction on Bayesian Networks . . . . . . . . . . . . . . 25

3.2 Areas of Application . . . . . . . . . . . . . . . 27

3.3 Theory of Bayesian Networks _. . . . . . . . . . . . 27

3.3.1 The Chain Rule for Bayesian Networks . . . . . . . . . . 28

3.3.2 Algorithms for Evaluating Bayesian Networks . . . . . . 28

3.3.2.1 Exact Inference Algorithms . . . . . . . . . . . 29

3.3.2.2 Approximate Inference Algorithms . . . . . . . . 32

3.4 Realization of Bayesian Network _. . . . . . . . . . . . 33

3.4.1 Nodes, Network and Structural Validation . . . . . . . . . 34

3.5 Quantifying the Network . . . . . . . . . . . . 36

3.5.1 Probability Elicitation . . . . . . . . . . 36

3.5.2 Protocols and Bias . . . . . . . . . . . . . . . 37

3.5.3 Validation of Estimates . . . . . . . . . . . . . . 39

3.6 Strengths and Limitations of Bayesian Networks . . . . . . . . . 40

3.6.1 Concluding Remarks . . . . . . . . . . . . . . . . . 41

4 Introduction to the Case Study 43

4.1 Introduction . . . . . . . . . . . . . . . 43

4.2 Problem Description . . . . . . . . . . . . . . . . . . . 43

4.3 Specific Objectives in this Case Study . . . . . . . . . . . . . . . . 44

4.4 Scope of Modeling and Decisions . . . . . . . . . . . . . . 44

5 Construction of a Bayesian Network for Foreign Exchange Set$\begin{array}{ll}\text { tlement } & 47\end{array}$

5.1 The Foreign Exchange and Money Market Settlement Process . . 47

5.2 Building the Network . . . . . . . . . . . . . . . . . . 48

5.3 Structural Model Building . . . . . . . . . . . . . . . . . . . . 49

5.3.1 Risk, Process Mapping and Nodes Identification . . . . . . 49

5.3 .2 Network Structure . . . . . . . . . . . . . . 51

5.3.3 Conditional Probability and Node Description Tables . . . 54 
5.3.4 Model Structure Validation . . . . . . . . . . . . . 57

5.4 Quantifying the Network . . . . . . . . . . . . . . 58

5.4 .1 Objective Data . . . . . . . . . . . . . . 58

5.4 .2 The Elicitation Process . . . . . . . . . . . . . . . . . 59

5.4.3 Protocol, Instruments and Procedures . . . . . . . . . . . 60

5.4 .4 Conducting the Elicitation . . . . . . . . . . . . . . . 64

5.4.5 Validating the Estimates . . . . . . . . . . . 67

5.5 Data Processing . . . . . . . . . . . . . . . . . . 69

5.5.1 Frequency Data . . . . . . . . . . . . . . . . 69

5.5.2 Loss Severity Data . . . . . . . . . . . . . 70

5.5.3 Loss Severity - Probability Computations . . . . . . . . . . 75

5.6 Aggregating the Frequency and Severity Outputs through MonteCarlo Simulation . . . . . . . . . . . . . . . . 78

5.7 Maintaining the network . . . . . . . . . . . . . 80

6 Results, Validation and Applications $\quad 81$

6.1 Model Results . . . . . . . . . . . . . . . . . . . . . . . 81

6.1.1 Results - Frequency of Failure and Severity of Losses . . . 81

6.1.2 Results - Aggregated Potential Losses . . . . . . . . . . . 83

6.1.3 Results - Operational Value at Risk . . . . . . . . . . . . . 84

6.2 Model Validation . . . . . . . . . . . . . . . . . . . 85

6.2.1 Comparison of Results - Frequency of Failures . . . . . . . 86

6.2.2 Comparison of Results - Severity of Losses . . . . . . . . . 87

6.2.3 Comparison of Results - Potential Losses . . . . . . . . . . 89

6.3 Managing Operational Risk with the Model . . . . . . . . . . . . 94

6.3.1 Managing the Drivers of Loss Severity . . . . . . . . . . 95

6.3.2 Managing the Drivers of Frequency of Failures . . . . . . . 101

6.3.3 Prediction of Operational Value at Risk . . . . . . . . 107 
7.1 Summary of Work . . . . . . . . . . . . . . . . . 109

7.2 Further Work . . . . . . . . . . . . . . . . . . 111

7.3 Conclusion . . . . . . . . . . . . . . . . . . . . 112

A Questionnaire

B Prior Probabilities 


\section{List of Figures}

2.1 Economic capital, unexpected and expected loss . . . . . . . . 16

3.1 An example of a Bayesian network . . . . . . . . . . . . 26

3.2 A BN flow diagram . . . . . . . . . . . . . . . 34

5.1 FX process flow. Source: The Foreign Exchange Committee, Federal Reserve Bank of New York . . . . . . . . . . . . . . . . . . . 48

5.2 Frequency of failure network . . . . . . . . . . . . 53

5.3 Loss severity network . . . . . . . . . . . . . . . . 54

5.4 Typical questionnaire format . . . . . . . . . . . . . 63

5.5 Exploratory graphs of some trade values . . . . . . . . . . . . 72

5.6 Kernel density estimation . . . . . . . . . . . . . . . 74

5.7 Assessing goodness of fit . . . . . . . . . . . . . 75

5.8 Aggregating frequency and severity distributions . . . . . . . . 78

6.1 Frequency network showing some posterior probabilities . . . . . . 82

6.2 Severity network showing some posterior probabilities . . . . . 83

6.3 Histogram of potential losses . . . . . . . . . . . . . . . . . 84

6.4 Comparison of frequency of failure . . . . . . . . . . . 87

6.5 Commulative distribution function of losses (logscale) . . . . . . 88

6.6 QQ plots of losses with truncations at 100, 500 and 1000 EUR . . 89

6.7 Potential loss - cdf comparison . . . . . . . . . . . . . . . 91

6.8 Potential loss - qq plots I (model versus historical data) . . . . . . 92

6.9 Potential loss - qq plots II (model versus historical data) . . . . . 93 
6.10 Illustration SI - largest loss amount . . . . . . . . . . . . . . . 96

6.11 Illustration SI - loss with days delay . . . . . . . . . . . . . . . . 97

6.12 Illustration SII - stop loss with most likely combination of states . 98

6.13 Probability curves for various days delays (daysdelay $=1$ is solid line, 2 is dash, 3 is longdash, 4 is dotdash and 5 is dotted). Vertical line is the 50m EUR trade value . . . . . . . . . . . . . . . . 100

6.14 Illustration SIII - days delay scenario . . . . . . . . . . . . . . 101

6.15 Illustration FI - back office system failure . . . . . . . . . . . . . . 102

6.16 Illustration FI - effect of trade volume on systems . . . . . . . . . 103

6.17 Illustration FII - causes of vulnerability to failure . . . . . . . . . 104

6.18 Illustration FII - effect of quality of SSI . . . . . . . . . . . . 105

6.19 Illustration FIII - most sensitive nodes . . . . . . . . . . . . . 105

6.20 Illustration FIII - most sensitive nodes . . . . . . . . . . . . . 107

A.1 Questionnaire introduction . . . . . . . . . . . . . . . 114

A.2 Questionaire introduction - bias . . . . . . . . . . 115

A.3 Questionnaire introduction - interview information . . . . . . . 115

A.4 Sample Questionaire - trade volume . . . . . . . . . . . 116

A.5 Sample Questionnaire - gross or netting settlement . . . . . . . 117

A.6 Sample Questionnaire - days delay (1) . . . . . . . . . . . 118

A.7 Sample Questionnaire - days delay (2) . . . . . . . . . . . . . 119 


\section{List of Tables}

5.1 Risk and process mapping . . . . . . . . . . . . . . . 50

5.2 Node description table - frequency of failures . . . . . . . . . . . 56

5.3 Node Description Table - severity of losses . . . . . . . . . . . . 57

5.4 Currency distribution and interest rates . . . . . . . . . 71

5.5 Monte-Carlo simulations . . . . . . . . . . . . . . . . . . . 79

6.1 Summary statistics of potential loss . . . . . . . . . . . 83

6.2 Operational risk unexpected loss . . . . . . . . . . . . 85

6.3 Operational risk unexpected loss . . . . . . . . . . . . . . . . 90

A.1 Matrix format questionnaire - Confirmation matching . . . . . . 120

A.2 Matrix format questionnaire - Number of settlement failure . . . . 121

B.1 Elicited probabilities - frequency of failure I . . . . . . . . . . . 124

B.2 Elicited probabilities - frequency of failure II . . . . . . . . . 125

B.3 Elicited probabilities - loss severity network . . . . . . . . . 126

B.4 Days delay node probabilities - with logit adjustments for illustration SIII in chapter $6 \ldots \ldots$. . . . . . . . . . . . . 126 



\section{List of Abbreviations}

$\begin{array}{ll}\text { ACI } & \text { International Financial Markets Association } \\ \text { AIB } & \text { Allied Irish Bank } \\ \text { AMA } & \text { Advanced Measurement Approach } \\ \text { BIS } & \text { Bank for International Settlement } \\ \text { BN } & \text { Bayesian Network } \\ \text { CFP } & \text { Committee for Professionalism - ACI } \\ \text { CLS } & \text { Continuously Linked Settlement } \\ \text { CPT } & \text { Conditional Probability Tables } \\ \text { CSA } & \text { Control Self Assessment } \\ \text { DAG } & \text { Directed Acyclic Graph } \\ \text { FX } & \text { Foreign Exchange } \\ \text { KRI } & \text { Key Risk Indicator } \\ \text { LDA } & \text { Loss Distribution Approach } \\ \text { MCMC } & \text { Markov Chain Monte - Carlo Simulation } \\ \text { MM } & \text { Money Market } \\ \text { NAB } & \text { National Australian Bank } \\ \text { NDT } & \text { Node Description Table } \\ \text { OpVaR } & \text { Operational Value at Risk } \\ \text { OR } & \text { Operational Risk } \\ \text { OTC } & \text { Over The Counter } \\ \text { SI } & \text { Settlement Instructions } \\ \text { SOX } & \text { Sarbanes Oxley Act } \\ \text { STP } & \text { Straight Through Process } \\ \text { VaR } & \text { Value at Risk } \\ & \end{array}$




\section{Currencies}

$\begin{array}{ll}\text { AUD } & \text { Australian dollar } \\ \text { BRL } & \text { Brazilian real } \\ \text { CAD } & \text { Canadian dollar } \\ \text { CHF } & \text { Swiss franc } \\ \text { CZK } & \text { Czech koruna } \\ \text { DKK } & \text { Danish krone } \\ \text { EUR } & \text { Euro } \\ \text { GBP } & \text { Great Britain Pound Sterling } \\ \text { HKD } & \text { Hong Kong dollar } \\ \text { HUF } & \text { Hungarian forint } \\ \text { JPY } & \text { Japanese yen } \\ \text { MXN } & \text { Mexican peso } \\ \text { NOK } & \text { Norwegian krone } \\ \text { NZD } & \text { New Zealand dollar } \\ \text { PLN } & \text { Polish zloty } \\ \text { RUB } & \text { Russian ruble } \\ \text { SEK } & \text { Swedish krona } \\ \text { SGD } & \text { Singapore dollar } \\ \text { SKK } & \text { Slovak koruna } \\ \text { TRL } & \text { Turkish Lira } \\ \text { USD } & \text { American dollar } \\ \text { ZAR } & \text { South African rand }\end{array}$




\section{Chapter 1}

\section{Introduction and Motivation}

\subsection{Introduction}

Recent financial scandals in the banking industry have caused considerable attention to be focused on operational risk. This is because an analysis of some of these scandals reveals that the underlying causes of these huge financial losses are due to Operational Risk (OR) and not to credit or market risk, as might initially appeared to be the case.

The Foreign Exchange (FX) market has had its fair share of these recent scandals. Two most recent examples of operational risk losses in the FX markets are the National Australia Bank's 227 million USD loss in 2004 and Allied Irish Bank's 750 million USD loss in 2002. These losses have had serious negative impact on the firms' profitability and reputation. Besides scandalous losses in the FX market, trade processing and settlement errors, as well as incorrect settlement, if not controlled, may lead to indirect costs such as compensation payments to counterparties, or to the development of large losses in a firm's portfolio due to managing the wrong position. Operational risk losses in the financial industry usually occur at the business unit level and are due to weak management oversight, weak internal controls or the lack of it, or to breakdown of proceedures among others. Operational risk therefore has to be managed at the business unit level.

This thesis is about managing OR at the business unit (micro) level. It concentrates on FX and Money Market (MM) settlement processes and uses Bayesian belief networks (BN) as a tool to model the causal relationships between risk factors and Key Risk Indicators (KRI) within the FX and MM settlement process. 
Such a causal model is able to help risk managers understand the drivers of losses, to make predictions and also to assess the effects of possible interventions.

\subsection{Motivation and Problem Description}

The development of operational risk models has evolved rapidly over recent years. There are basically two reasons to this. One is external; banking regulatory compliance (Basel II Accord is coming into effect in late 2006) and the other is internal; most banks are realising that good operational risk management is a sound business practice. The influence of regulatory compliance is currently the greater of the two. Consequently, managing OR as done in banks is presently at the "macro", or top level and banks are more concerned, as it were, with getting their operational risk capital models approved by banking regulators. This trend is expected to continue for some time.

It is envisaged that after banks have achieved their first target of capital computation, their attention will be turned to managing operational risk at the "micro" or business unit level. It is at the micro level that operational risk losses actually occur. This makes managing OR at the business unit level the next logical stage of OR development. With this shift, emphasis will consequently be placed on models used for managing OR at the business unit level. Presently, models for managing operational risk at the business unit level are the Key Risk Indicators and causal models (for example, multifactor models and Bayesian networks) among others. Key Risk Indicators are the most widely used whereas the causal models are not well studied and documented.

A Bayesian belief network, is a tool which can relate and show the causal relationship among risk factors, key risk indicators and some operational risk attributes. Such a causal model is very useful in managing OR at the business unit level since it can perform predictions under various scenarios, that is, perform "whatif-analysis" and show the results of possible interventions immediately. Alexander (2003), King (2001) and Ramamurthy et al. (2005) have made some attempts at demonstrating the usefulness of $\mathrm{BN}$ in managing OR at the business unit level. Other researchers like Giudici (2004) and Neil et al. (2005) however, emphasize $\mathrm{BN}$ as a tool for computing economic capital.

Significantly missing in the available literature on OR is a complete practical guidance on how a BN can be implemented in a real-world situation from the point of realising the network structure, through probability elicitations and managing OR with the model to maintain the BN. The absence of this detailed procedural 
guide has lead to OR practioners, who might agree on the usefulness of $\mathrm{BN}$, to conclude that $\mathrm{BNs}$ are too complex to construct and maintain, and that gives insufficient return for the effort. This is what has contributed to the under utilization of Bayesian network technology in operational risk management although BNs have had great successes in other fields of study. This thesis addresses these issues and, in particular, provides an illustration of how one can go about applying Bayesian networks in a practical setting.

\subsection{Objectives of Research}

The objectives of this thesis are

1. to provide a complete practical procedural guidance on how a BN can be implemented as a causal model for the purpose of managing operational risk in a specific business line or business unit.

2. to re-affirm the usefulness of a BN and also to demonstrate how a BN can be used to manage OR in a real - world situation.

These objectives are achieved through 1) the construction of a BN for FX and MM settlement process in a bank. The BN establishes the causal relationship between the necessary risk factors, key risk indicators and other operational risk attributes; and 2) the application of the network to FX and MM process to assist in understanding the drivers of operational risk and the effects of possible interventions, and to compute an economic capital for OR for internal management purposes.

\subsection{Outline of the Thesis}

This thesis is divided into two parts; Part I and Part II. Part I is captioned "Background, Literature and Available Approaches" and includes Chapters 1, 2, and 3. Chapter 1 introduces and motivates the thesis, and sets out the objectives of the thesis. Chapter 2 provides the background to operational risk, foreign exchange and money markets, and reveiws the available approaches to quantifiying and managing operational risk. It ends with the assertion that Bayesian networks provide a powerful tool for managing operational risk. The theory behind Bayesian networks, and how they are realized (including the elicitation of expert opinion) is presented in Chapter 3. 
Part II is a case study titled "Application of BN to FX and MM settlement". It shows a practical application of BN to FX and MM settlement. It starts with defining and establishing the objectives of the $\mathrm{BN}$ in Chapter 4 and proceeds to how the BN model is constructed and quantified in Chapter 5. Chapter 6 shows the results of applying BN model to FX settlement and also illustrates how the model can be used to manage operational risk. The summary and conclusions of the thesis is given in Chapter 7. 


\section{Chapter 2}

\section{Operational Risk, Foreign Exchange and Money Market}

\subsection{Basel II, Operational Risk and Sarbanes- Oxley}

The Basel Committee on Banking Supervision (The Committee) of the Bank of International Settlement has been working for several years on the New Basel Accord, known as Basel II to replace the old Accord, known as Basel I, which was published in 1988. In June 2004, the Committee published the much awaited "Basel II" framework for bank capital adequacy. The basic framework will become available for implementation by national banking supervisors towards the end of 2006 , and the advanced versions of the rules will be implemented by the end of 2007.

The Basel Accords sets standards on how risk is measured and the capital which regulators require banks to hold for the risks they take. The current consensus is that banks face three types of risks - market, credit and operational. Basel I focused on market risk and some specifications for credit risk. It did not recognise credit risk mitigation among others. Significantly, Basel I did not have any Operational Risk requirements. Basel II is an improvement of Basel I and also a reflection of the current consensus. It is also seen as a response to the huge financial losses that some banks, like Barings, Sumitomo, National Westminster and Bank of New York among others, have suffered within the last two decades. Basel II hinges on three pillars:

- Pillar 1 concentrates on the minimum capital requirements of Basel I and 
introduces a new specific charge for Operational Risk;

- Pillar 2 - Supervisory review processs; supervisors should review banks' internal capital adequacy, take actions where necessary, ensure that banks operate above the minimum regulatory capital ratios, and require rapid remedial actions if capital is not maintained or restored.

- Pillar 3 - More public disclosure to the market; banks must disclose their strategies and processes for managing risk, risk management structure and organization, scope and nature of risk reporting, policies for hedging and mitigating risk, monitoring of effectiveness of risk mitigants and their approaches to regulatory capital assessments.

Operational Risk has been defined by the Basel Committee on Banking Supervision as

the risk of loss resulting from inadequate or failed internal procedures, people, systems or from external events (Basel Committee on Banking Supervision, 2001).

This definition includes legal risk, but excludes strategic and reputational risk. Operational Risks, unlike market and credit risk are specific to the factors and circumstances of each institution. The factors that drive Operational Risk are internal in nature, which includes a firms specific processes, culture, personel, and technology. In addition Operational Risk is dynamic - it continuously changes with business strategy, processes, technology and competition.

A firm's Operational Risk exposure can increase as a result of poorly trained, overworked or unmotivated employees, complex or poorly designed systems or processes, which are either unfit for use, or malfunction, and also external events like the attack on the World Trade Centre on September 11, 2001. Failure to adequately manage Operational Risk can lead to the disruption and continuity of a firm's business activities. It is a known fact that the real underlying causes of losses that lead to failures of many financial firms are operational, even though the immediate cause of these losses appeared market or credit related.

Basel II provides three approaches: the Basic Indicator Approach, the Standardized Approach, and the Advanced Measurement Approach for calculating Operational Risk capital charge in a continuum of increasing sophiscation and risk sensitivity. Banks are encouraged to move along the spectrum of available 
approaches as they develop more sophiscated Operational Risk measurement systems and practices. These approaches, as contained in the Committee's 2003 Operational Risk-Rules Language (Basel Committee on Banking Supervision, 2003) are summarized below.

The Basic Indicator Approach calculates the required capital on the basis of a fixed percentage (denoted alpha) of average annual gross income over the previous three years. Alpha is currently set at $15 \%$ by the Committee. No qualifying criteria are set out in the rules for usage of this approach. However banks using this approach are encouraged to comply with the Committee's guidance on Sound Practices for the Management and Supervision of Operational Risk (Basel Committee on Banking Supervision, 2002)

The Standardized Approach also calculates the required capital on the basis of a fixed percentage of average annual gross income but uses different indicators (denoted betas) for each predefined business line. A banks activities are divided into eight business lines, namely corporate finance, trading and sales, retail banking, commercial banking, payment and settlement, agency services, asset management and retail brokerage. Gross income here refers to the gross income of the business line and the total charge is a simple summation of the regulatory capital charge across each of the business lines. The various betas so far proposed range between $12 \%$ to $18 \%$ (Basel Committee on Banking Supervision, 2003).

The Advanced Measurement Approach is based on a risk measure generated by a bank's own internal Operational Risk measurement systems using some qualitative and quantitative criteria set out by the Committee. This approach gives banks the flexibility to develop their own Operational Risk measurement systems, which may be verified and accepted for regulatory purposes. Some of the qualifying criteria for the use of this approach include tracking of internal loss data, the use of relevant external data, the use of scenario analysis or expert opinion in conjunction with external data to evaluate exposure to high severity events, and assessment of key business envrionment and risk control that changes the Operational Risk profile. Under this approach the risk mitigation impact of insurance is recognised.

\section{Sarbanes-Oxley}

The new Sarbanes-Oxley (SOX) Act of 2002 is concerned with corporate governance and is intended to restore investor confidence, protect investors and safeguard public interest, especially after some of the recent scandals, for example the Enron scandal in 2001. SOX applies to all public corporations in the USA. 
Some sections of the Act (Sections 404 and 409) like the Basel II Accord, deals with assessment of internal controls and real-time issuer disclosures.

Section 404, for example requires each annual report to contain an internal control report. The idea behind the report is to state management's responsibility for creating and maintaining an adequate control structure and proceedures for financial reporting, and also to assess the structure and procedures in place. Like Pillar II of the new Basel Accord, Section 409 of SOX also requires the timely public disclosure of material changes in financial conditions or operations.

SOX and Basel II have some similar objectives, and most financial institutions are using a common type of framework and governance model to respond to these regulatory requirement. It is thus common that the same unit within a bank is responsible for Operational Risk and corporate governance.

\subsection{The Foreign Exchange and Money Markets}

The Foreign Exchange Market (FX) is the largest and most liquid financial market in the world. The FX market has a turnover that averages 1.9 trillion USD per day in the cash exchange and an additional 2.4 trillion USD per day in the overthe-counter (OTC) FX and interest rate derivatives market in April 2004 (Bank for International Settlement, 2005). The importance of the FX market cannot be overemphasied. It has an enormous impact on the global economy and affects trading of goods, services and raw materials throughout the world.

Although the FX market is arguably the largest market (it's average volume of trading is larger than the combined volumes of all the world's stock markets) it is the least regulated since it cuts across every boarder, and regulating it is nearly impossible. Unlike the stock market, currencies are traded without the constraints of a central physical exchange. Much of the trading is conducted via telephone, computer networks and other communication means. It is a 24hour-per-day market during the business week all around the world and spans all continents.

There are four major types of participants in FX market: banks, commercial dealers, private investors and central banks. Approximately two-thirds of all FX transactions are handled by banks trading with each other. The major centers of FX inter-bank trading are London, New York, Tokyo, Singapore, Frankfurt and Zurich (Bank for International Settlement, 2002). Commercial dealers are primarily institutions involved in international trade that require foreign curren- 
cies in the course of their businesses. Private investors are those who find the market attractive and thus capitalize on the market traits. Central banks, representing their government, buy and sell currencies as they seek to control the money supply in their respective countries.

FX transactions come in various forms. Spot transactions are usually based on currency rates quoted for two-day settlement. Eceptions are the US dollar and Canadian dollar that are traded for one-day settlement. Forward FX agreement specifies a currency exchange rate used for delivery at a stated time or value date, in the future. Other forms are currency swap transactions and options on inter-bank FX transactions. In 2004 Spots accounted for about 35\% of the global turnover, Outright forwards 10\%, and FX swaps 50\%, the rest being estimated gaps (Bank for International Settlement, 2005).

Currencies are traded in pairs. This involves the simultaneous purchase of one currency while selling another currency. The most heavily traded currencies, which accounts to about $85 \%$ of the toal transactions, are the so called "major" currencies including the US dollar, Euro, Japanese yen, British pounds, Swiss franc, Canadian dollar and the Austrialian dollar. In 2004 USD/Euro accounted for $28 \%$ of global turnover followed by USD/Yen 17\%, USD/Sterlin 14\%, USD/Swiss Franc 4\%, USD/Canadian dollar 4\%, USD/Australian dollar $5 \%$, USD/other 16\% and Emerging currencies 5\% (Bank for International Settlement, 2005).

The Money Market (MM) generally refers to borrowing and lending for periods of a year or less. Like the FX market, the money market has no specific location and is primarily a telephone market. It emcompases a group of short-term credit market instruments, future market instruments, and the central banks' discount window. MM instruments are generally characterized by a high degree of safety of principal and are most commonly issued in units of 1 million USD or more. Maturities ranges from one day to one year; the most common being three months or less.

Money markets arise because receipts of economic units do not usually coincide with their expenditures. Holding enough balances to cushion this difference comes with a cost in the form of foregone interest. To minimize this cost, economic units prefer to hold the minimum money balances needed for the day-to-day transactions and supplement these balances with holding MM instruments that can be converted to cash quickly at relatively low cost. Short-term cash demands are also met by maintaining access to the MM and raising funds when required. The 
principal players in MM are commercial banks, governments, corporations, MM mutual funds, brokers, dealers, government sponsored enterprises, and futures market exchanges.

Transactions involving FX and MM need to be settled (exchange of value between the parties of the trade) after they have been made. A detailed description of FX and MM settlement process is given in Chapter 5. There are several risks involved in the settlement process. Prominent among them are settlement risk and Operational Risk. Operational Risk is addressed in the next Section.

Settlement risk, also referred to as Herstatt risk, in FX transactions is the risk of loss when a party to a trade pays out the currency it sold but does not receive the currency it bought. This is on the premise that FX trades are usually settled by making two separate payments. The separation of the two currency legs creates settlement risks. Industrial efforts have recently been made to eliminate the prinicipal risk associated with FX settlement. One such effort is the establishment of the Continuously Linked Settlement (CLS) Bank ${ }^{1}$. CLS eliminates settlement risk by simultaneously settling the two currency legs of a transaction across the books of CLS Bank. Both sides of a trade are either settled or neither side is settled.

\subsection{Operational Risk in Foreign Exchange and Money Market}

Operational Risk in FX and MM usually involves problems with processing, product pricing and valuation. It may also come from poor planning and procedures, inadequate systems, failures to properly supervise staff, fraud and human error (Foreign Exchange Committee, 2001). To manage OR in the FX market, firms must plan and implement procedures, processes and systems that will ensure that proper controls are in place and constantly monitored.

Operational Risk in FX, if not managed, can have serious negative impact on a firms profitability and reputation. FX trade processing and settlement errors as well as incorrect settlement may lead to indirect costs, such as compensation payments to counterparties or the development of large losses in a firm's portfolios due to managing the wrong position. Additional cost may also be incured from investigating or negotiating a solution with a counterparty (Foreign Exchange

\footnotetext{
${ }^{1}$ see www.cls-group.com
} 
Committe, 2003).

Two most recent scandals in the FX and MM market that resulted in huge losses are the 1) U.S unit of Allied Irish Bank (AIB) with losses amounting to 750 million USD uncovered in February $2002^{2}$ and 2) National Australia Bank's (NAB) rogue deal, uncovered in January 2004, that cost the Bank 277 million USD $\operatorname{losses}^{3}$. These two scandals, although surpassed in magnitude by Nick Leeson's 1.2 billion USD loss that collapsed Barings bank in 1995, and Yasuo Hamanaka's 2.6 billion USD loss uncovered in 1996 at Sumitomo, may stand out to the largest so far in the FX market. A detailed description of the AIB and NAB scandals is given below.

\section{Allied Irish Bank's 750 million USD Scandal}

The AIB scandal involved a currency trader in the person of John Rusnak who executed a large number of foreign exchange transactions involving spot and forward contracts. The trader appeared to have offset the risk involved in the trasaction by taking out option contracts, which is the usual practice. The bank later discovered that the losses on the spot and forward contracts had not been offset by profits from the options deals. In addition he created fake options and found ingenious ways of getting these fiticious orders into the banks books.

Although the scandal did not cause AIB to collapse, it was large enough to cause AIB to lose $60 \%$ of it's 2001 earnings. The scandal subsequently resulted in a takeover of AIB by M and T Bank Corporation in April 2003. The major question about this scandal, like many others, is why the bank's internal controls failed to spot the fraud.

According to an independent report commissioned by the AIB board commonly referred to as the "Ludwig report" (see Ludwig, 2002) Rusnak took advantage of a gap in the control environment, namely a failure in the back office to consistently obtain transaction confirmations. There were also flaws in the architecture of Allfirst's trading operations and a lack of good oversight from senior management in Dublin and Baltimore on Allfirst's proprietary trading operations.

\section{National Australian Bank's 27r million USD Scandal}

In the NAB scandal, four of the bank's traders, three in Melbourne and one in London, had mistakenly speculated that the Australian and New Zealand

\footnotetext{
${ }^{2}$ see http://news.bbc.co.uk/2/hi /business/1807497.stm, visited 19.07.2005

${ }^{3}$ see http://edition.cnn.com/2004/BUSINESS/01/26/nab.forex /index.html, visited 19.07.2005
} 
currencies would fall against the U.S dollar. These traders not only guessed wrongly but also exploited the weaknesses in the bank's internal procedures and breached trading limits that led to the losses.

The Committee for Professionalism (CFP) of the International Financial Market Association (ACI) made an important statement after these two scandals which can be broken down into two parts. The first is to the effect that frauds and misdemeanors are usually perpetrated through a simple exploitation of lax controls throughout the transaction flow process, such as poor confirmation details checking, or poor oversight by a firm's management. The second stresses that ultimate responsibility for frauds and misdemeanors must rest with senior management who should ensure that the systems and controls in place within their organisations are robust enough to swiftly identify erroneous trades or employee wrongdoing.

These two events are the sensational ones that made the headlines but there are several instances of million dollar losses which were not reported so prominently. An analysis of these two incidents shows the need for effective controls and tight procedures in the 1.9 trillion a day FX market. Effective controls and strong management oversight are needed, not only for the big investment banks but most importantly for the mid-sized banks that have often been overlooked. Controls and management oversight should span the entire FX transaction process of pre-trade preparation, trade capture, confirmation, netting, settling and nostro reconciliation.

\section{Attempts to Manage Operational Risk in Foreign Exchange}

Some attempts have been made to reduce the incidence of Operational Risk within the FX markets both before and after these debacles. One of such attempt is the Foreign Exchange Committee of the Federal Reserve Bank of New York's best practices for managing Operational Risk in foreign exchange (see Foreign Exchange Committe, 2003). This document was first published in 1996.

The document is a collection of best practices that may help to mitigate some of the Operational Risk specific to the FX industry. It provides best practices for each of the seven steps of FX trade process flow 1) pre-trade preparation 2) trade capture 3) confirmation 4) netting 5) settlement 6) nostro reconciliation and 7) accounting/financial control processes. It concentrates on the most vulnerable areas to Operational Risk and provides a list of best practices specific to that area. Most of the best practices in the document is already in use by the working 
group members responsible for the document. Some electronic trading platforms ${ }^{4}$ have been developed on the basis of the Federal Reserve Bank of New York's best practices for managing Operational Risk in foreign exchange.

\subsection{Review of Operational Risk Methods}

The development of techniques for the measurement and management of OR is very dynamic. There are, however, some common emerging practices among several banks. The dynamic nature of the techniques involved may be due to two reasons: the fact that formal Operational Risk management is at its infant stages and the fact that the Basel Accord permits a substantial degree of flexibility (within the context of some strict qualifying criteria) in the approach used to asses capital requirements, especially within the Advanced Measurement Approach.

Measurement and management of OR faces many challenges, which include: the relative short time span of historical loss data, the role of internal control environment and its ever changing nature, (which makes the historical loss data somehow "irrelevant") and the important role of infrequent, but very large loss events. These challenges are the drivers of the various approaches in practice.

The review of OR methods tries to separate models that are used for quantifying OR and calculating Economic capital from those that are used for solely management purposes. This demarcation is however, very difficult to do since the models that are used for quantifying OR can also be used for managing OR. What is actually done is to rather separate models that are used for managing OR internally from those that are used for calculation of economic capital for regulatory purposes. We call the first group of models "models for managing OR" and the second group "models for quantification and capital allocation".

\subsubsection{Models for Quantification and Capital Allocation}

There are basically three different models for quantifying and allocating capital within the Advanced Measurement Approach. These are the Loss Distribution Approach (LDA), the Scorecard or Risk Drivers and Control Approach, and the Scenario-based Approach. The different measurement approaches have common elements among them since all are structured around the four basic elements of an Advanced Measurement Approach, namely internal data, external data,

\footnotetext{
${ }^{4}$ see for example FXall at www.fxall.com
} 
scenario analysis and factors reflecting the business environment and internal control system.

The main differences among these approaches are the differences in emphasis of the common elements. The Loss distribution approach emphasizes the use of internal loss data, the Scorecard approach emphasizes the assessment of business environment and internal control systems, and the Scenario-based approach uses various scenarios to evaluate an organisation's risk. In spite of the differences in emphasis, in practice most banks are attempting to use elements of all three approaches.

One common feature of these methods is the way external data is sourced and used. External data is used to supplement internal data, especially at the tails of the loss distribution. Banks are either internally collecting external data, using commercial vendors or industry data pools. In order to use only relevant external data in their models, banks segment external data into peer groups and use corresponding data from groups to which they belong. In addition, expert judgement on individual data point is sought to determine if, from the perspective of the bank, the point is an outlier to be excluded. Furthermore, external data is scaled before usage in a particular bank. This is based on the assumption that OR is dependent on the size of a bank. A way of dealing with scalability is the use of regression analysis to determine the relationship between size and frequency, and also the relationship between severity of losses and size.

\subsubsection{Loss Distribution Approach}

The Loss Distribution Approach (LDA) starts on the knowledge that loss data is the most objective risk indicator currently available, which is also reflective of the unique risk profile of each financial institution. Loss data is thus used as the primary input to creation of a loss distribution. LDA is cognisant of the inherent weaknesses of internal loss data and addresses these weaknesses. These include the fact that loss data provides only a "backward looking" measure and thus does not necessarily capture changes to the current risk and control environment and secondly loss data is not always available in sufficient quantities in any one financial institution to permit a reasonable assessment of exposure. The LDA addresses these weaknesses by integrating other AMA elements like external data, scenario analysis and factors reflective of the business environment and the internal control system.

The LDA uses standard actuarial techniques to model the behaviour of a firm's operational losses through frequency and severity estimation to produce an objec- 
tive estimate of both expected and unexpected losses. It starts with the collection of loss data and then partitions loss data into categories of losses and business activities, which share the same basic risk profile or behavoiour patterns. This is followed by modeling the frequency of losses and severity of losses separately, and then aggregating these distributions through Monte Carlo simulations, or other statistical techniques to obtain a total loss distribution for each loss type or business activity combination for a given time horizon.

Some common statistical distributions in use are the Poisson, negative binomial, Weibull and Gumbel distributions for frequency of failure and lognormal, lognormal gamma and gamma distributions for the severity of losses. The last step is to fit a curve to the total loss distribution obtained and to check the goodness of fit through standard statistical techniques like the Pearson's Chi-Squared test and the Kolmogorov-Smirnov test. Further details of the LDA approach is given in Industry Technical Working Group (2003).

Under the new Accord, banks are required to calculate the economic capital. This is true for all three approaches under the AMA. The difference between the loss value corresponding to the percentile in the tail and the mean of the total loss distribution is the Economic Capital for the chosen percentile. The new Accord presently sets the percentile at $99.9 \%$. The mean is called the expected potential loss and the difference between the percentile value and the expected loss is called the unexpected loss (see Figure 2.1). It is this loss that banks are required to cushion themselves against. 


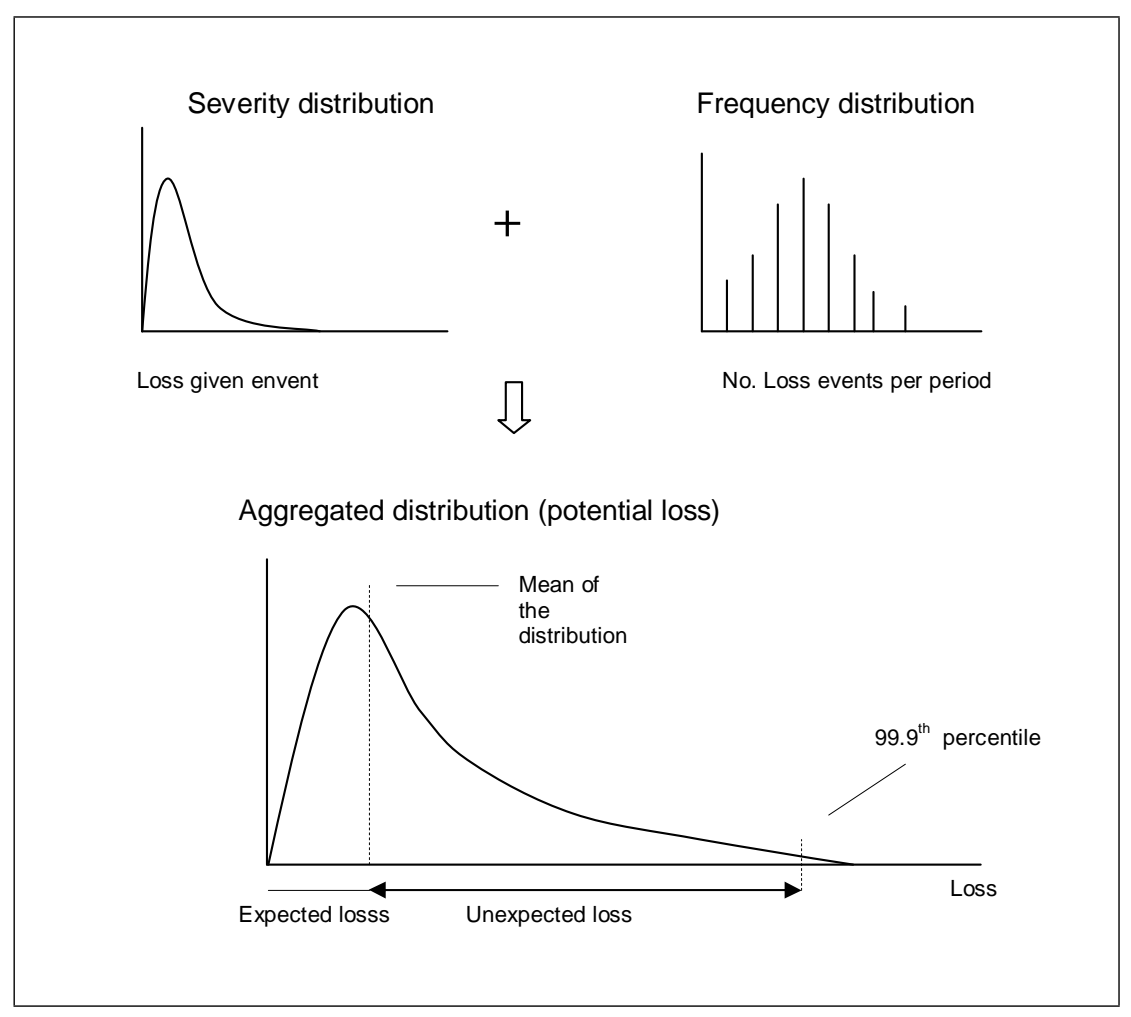

Figure 2.1: Economic capital, unexpected and expected loss

As mentioned earlier, scenario analysis as an element of the AMA is incorporated into the LDA. The purpose of incorporation is to 1) supplement insufficient loss data, 2) provide a forward-looking element in the capital assessment and 3) stress test the capital assessment.

Supplementing insufficient loss data is usually done at the tails of the distribution. One way of doing this is to create some scenarios such as Expected loss (optimistic scenario), Unexpected serious case loss (pessimistic scenario), Unexpected worst case loss (catastrophic scenario) for a potential loss event. Experts are then asked to estimate the probability of occurrence and the severity of losses for each of these scenarios. The weighted average loss of the potential event under the three scenarios, where the weights are corresponding probability of occurrence is computed and added as a data point to the historical loss data. Another way is to derive distribution parameters from the scenarios that can be combined with similar parameters from historical data to generate the capital level.

To provide a forward looking element in the capital assessment, distribution parameters of the historical data are modified by the estimates given by the experts 
in the scenario analysis. The weighting given to the parameters from the historical data and scenarios are a reflection of the degree of confidence attached to each of them. Bayesian methods can also be used to combine the two sets of information. In stress testing experts are basically asked to provide estimates for "stress scenarios" and the estimates are used as inputs to calibrate the parameters of the capital quantification model.

\subsubsection{The Scorecard or Risk Drivers and Controls Approach}

The scorecard approach also known as the Risk Drivers and Controls approach directly connects risk measurement and the Operational Risk management process. Within the financial industry, the scorecard methodology refers to a class of diverse approaches to Operational Risk measurement and capital determination. The core of these approaches is the assessment of specific Operational Risk drivers and controls. These approaches not only quantify the risk faced by organisations but also quantifies the controls used to mitigate those risks. The scorecard approach has the advantage of providing an increased understanding and transparency of OR exposure and the control environment.

The Scorecard approach is questionnaire based and focuses on the principal drivers and controls of Operational Risk across an Operational Risk category. The questionnaires are designed to probe for information about the level of material risk drivers and quality control. Other key features of this approach are transparency to line managers, responsiveness to change (risk profile and business mix), behavorial incentives for improved risk management (for line managers), and forward looking.

Although the Scorecard approach relies heavily on control self assessment, historical loss data do play a role in this approach. Historical data can be used in 1) identifying drivers and mitigants of risk categories, which is necessary for formulating specific questions and responses, 2) determining the initial level of OR capital, 3) generating OR scenarios for high impact events and 4) cross checking the accuracy of questions in the responses and challenging the existing scorecards in terms of impact and likelihood.

A capital amount is generated with the Scorecard approach by either running simulations or using the approach of initial capital determination. To run simulations, values are given (through the risk and control assessments) to the elements of the scorecard such as the percentage of occurrence of the risk likelihood, a monetary value for the risk impact and percentage of control failures. With this information, and incorporating some correlation values, three kinds of simulations 
can be run: simulate the control first and if a control fails, then simulate the risk; simulate the risk first and, if a risk happens, then simulate the controls; simulate both risk and controls. Details of these simulations can be found in Blunden (2003).

The initial capital determination approach starts with establishing an OR captial level or pool for a business unit or the entire organisation. Methods used to establish this level include the LDA, Standardized approach or the use of Benchmarks (proportion of total capital, peer institutions, capital for other risk types). Having determined the initial capital, there are two ways of distributing the capital. The first is termed "initial capital distribution" and the second "on-going capital distribution".

In initial capital distribution, the initial capital established earlier is distributed in a "top-down" process. It is allocated to the risk categories by a process which takes into account the historical data and qualitative information from the risk drivers and control obtained from the administered quesitionnaire. The quesitionnaire assesses both a risk score (profile) and appropriate scalar for a particular risk within each risk category for each business unit. The risk score and scalar are then used as factors for the capital distribution.

On-going capital distribution is a "bottom-up" process. Here the new capital allocation for each business unit is determined as a direct result of the changes in the risk score and risk scalars. That is to say, as the business progresses, the risk scores and scalars may change with time. These new (changed) factors are then used as distribution factors. The new OR capital for each business unit is therefore the sum of each risk category's new amount (due to changes in the risk scores and scalars) and the new OR capital for the entire organisation is the sum of all the capital amounts in all business units taking into account the relevant correlations that may be present.

\subsubsection{Scenario-Based Approach}

The Scenario-Based Approach of the AMA places scenarios at its center. It also draws on other available information such as expert experience, internal and relevant external historical loss data, key Operational Risk indicators and the quality of the control environment. This approach is sometimes seen as one that bridges the gap between the loss distribution and scorecard approaches.

The scenario based approach creates a forward-looking risk management framework, which is able to respond quickly to the business environment. Additionally, 
there is an important flow of management information during the assessment of various scenarios. This approach has been argued to be conceptually sound since only information relevant to the Operational Risk profile of a firm is input into the capital computation model. Additionally, the process is supported by a sound and structured organisational OR framwork and by an adequate IT infrastructure.

There are six key features involved in the Scenario-based approach. These include scenario generation, scenario assessment, data quality assessment, determination of the parameter values, model application and model output. Scenario generation deals with determining the scenarios for the assessments. The scenarios should be such that they capture all material risk and can be applied consistently across the firm. Scenario generation is done through the identification of risk factors by experts. Scenario assessment deals with estimating the loss frequency and severity for a specific scenario. The assessment is carried out by experts based on a combination of their industrial experience, insurance cover in place, key risk indicators, historical losses, and the quality of the relevant risk factors and the control environment.

The quality of the scenario assessment estimates is checked to ensure that it reflects the Operational Risk profile. Checking may be done by comparing actual losses against the experts' estimates. This is usually done by the internal audit department. The parameter values of the distributions to be used in the model are determined from the scenario assessments. This is done separately for the frequency and severity distributions across risk categories and business units. Model application is usually carried out by using Monte-Carlo simulation techniques to combine all the individual distributions per scenario class, across risk categories and business units to obtain an overall aggregated total loss distribution. The level of capital is derived from this total distribution. Further details of the Scenario-based AMA is given in Scenario Based AMA Working Group (2003).

\subsubsection{Models for Managing OR}

Apart from the models used for quantifying (sometimes also used for managing) OR described earlier, there are certain groups of models which are used specifically for managing OR internally within an organisation. Key Risk Indicators (KRIs) and causal models belong to this group.

The factors that drive an organisation's Operational Risk are mostly internal (people, process, systems) unlike those that drive market or credit risk. The internal nature of these factors mean that an organisation can control them to some 
extent. An organisation can therefore construct models that link the risk factors to certain Operational Risk attributes and either use these models to track occurrence of Operational Risk events for prompt actions, as done in KRIs or establish a cause-effect relationship (causal modeling) for managing OR. KRIs and causal models usually complement the quantification models thus giving an organisation a complete set of tools for quantifying and managing OR.

\section{Key Risk Indicators}

Key Risk Indicators are a part of the risk self assessment approaches and are used to manage OR. KRIs are regular measurements based on data, which indicate the operational risk profile of a particular activity or activities. They are selected to track near-real-time objective data on bank operations and also provide a forward looking measure of risk that is tied to managment. KRIs serve as early warning systems that can signal management when to act. They are used in the context of both "preventive" and "detective" controls. Some Operational Risk analysts are of the view that if KRIs had been in place, they could have raised a red flag for senior management in NAB's 360 million AUD loss in early 2004 since there were some 750 currency option limit breaches in just one month before the loss event.

The challenge with KRIs is the selection of the most relevant statistics to construct the KRIs. KRIs should be easily quantifiable measures (for example, transaction volume or growth in the number of unconfirmed trades in settlement) and threshold levels should be set for them to facilitate management response. Selection of KRI is usually done through self assessments, and interviewing executives and managers. Thresholds could be set at green, yellow and red with each threshold associated with some measurable quantity. Green could indicate risk are properly controlled, yellow that risks are approaching unacceptable levels, and red that risks have exceeded the acceptable level.

KRIs should be updated periodically to maintain their relevance because some become obsolete after some time; since a KRI is often selected because it tracks operational weakness so management is able to correct the weakness it tracks.

At the moment there are countless potential KRIs in the industry and there is an on-going exercise to develop a KRI framework for the banking industry ${ }^{5}$. The aim of the exercise is to achieve standardization, completeness and consistency, in order to create comparability and to enable aggregation, analysis, and reporting at the corporate level, which in turn will set the stage for real improvements in

\footnotetext{
${ }^{5}$ see www.kriex.com
} 
the effectiveness of KRIs.

\section{Causal Models}

Operational Risk causal models include Multifactor models, Bayesian or Causal networks, Fuzzy logic and Neural networks. OR causal models are management tools used for predicting various courses of action and intervention.

Multifactor modeling, as applied to OR is essentially a regression model in which the explained variable is an Operational Risk attribute being monitored and the explanatory variables are risk factors. With such a model one can track the effect that changes in the risk factors (causal factors) has on the Operational Risk attribute in question. Cruz (2002) applied multifactor modeling to explain Operational Risk losses. In this model the explained variable is operational losses and the explanatory variables are control environment factors, namely system downtime, number of employees, data quality and the total number of transactions. The model can be used to predict the Operational Risk value by varying any of the control environment factors.

Multifactor modeling, as described, is able to model the cause-effect relationship only at a single level (for example, $A$ depends on $B$ and $C$ ) and not several levels (for example, $A$ depends on $B$ which depends on $C$ and so on). Care should be taken not only in constructing or defining the relations between the explained and explanatory variables but also in using multifactor models. This is because it is possible to interprete associations as causalities and base actions or interventions on these associations. Some formal definitions of association and causality can be found in Pearl (2000).

Bayesian or causal networks (BN) have been used for quantifying and allocating capital in Operational Risk (Neil et al., 2005). Commercial software packages based on Bayesian networks for quantifying and allocating Operational Risk capital are also available ${ }^{6}$. Alexander (2003) and King (2001) illustrated the use of Bayesian networks for managing Operational Risk. Alexander (2003) further illustrated how the cost-benefit analysis of risk controls and interventions can be assessed by augmenting Bayesian networks with decision and utility nodes (Bayesian decision networks).

$\mathrm{A} \mathrm{BN}$ is able to tie all the four essential elements of the AMA (internal data, external data, scenario analysis and control environment) together. Using a BN to determine causal relationships and managing Operational Risk offers many advantages over traditional methods of determining causal relationships. These

\footnotetext{
${ }^{6}$ see some examples at www.agena.co.uk and www.algorithmics.com
} 
advantanges are discused in the next Section.

Some attempts have been made to use fuzzy logic and neural networks to quantify and manage Operational Risk (see, e.g, Cruz, 2002; Perera, 2000). These approaches, in contrast to BNs are representations of reasoning processes. The arrows or arcs in fuzzy logic and neural networks represent the flow of information during reasoning as opposed to Bayesian networks, which represents real causal connections (Pearl and Russel, 2001). Perhaps it is this reason that has made fuzzy logic and neural networks less attractive to Operational Risk practioners.

\subsection{Assessment of Bayesian Networks for Oper- ational Risk Management}

The changing emphasis of Operational Risk from economic capital computation to managing at the business unit level is likely to cause efforts to be concentrated on causal modeling. BN stands out as the model of choice for causal modeling at the business unit level and can be seen as an extention of the widely used KRI since it is able to relate KRIs to risk factors and other Operational Risk attributes. Despite BN's enormous potential it is also subject to criticism. An outlook of the prospects and criticism of $\mathrm{BN}$ in the context of $\mathrm{OR}$ management is given below.

\subsubsection{Prospects of Bayesian Networks for Operational Risk}

As a tool for managing OR at the business unit level, BN enjoys several advantages over other models. These include the following:

1. A BN is able to incorporate all the three essential elements of the AMA outlined earlier (internal data, external data, scenario analysis, and factors reflecting the business environment and control systems) into one simple model that is easy to understand. Unlike the other models reviewed earlier, a $\mathrm{BN}$ is able to place equal emphasis on all the essential AMA elements.

2. A BN can be constructed into a "multi-level" model which can show several levels of dependency among several risk factors (e.g. frequency of failure could depend on the IT systems in place which inturn depends on the transaction volume). In contrast, multifactor models can show only one 
level of dependency. This means that a $\mathrm{BN}$ can be used to manage the risk involved in the detailed process of a business unit

3. When extended into a decision network a $\mathrm{BN}$ can provide a cost-benefit analysis of risk controls, where the optimal controls are determined within a scenario analysis framework (Alexander, 2003)

4. BNs present a natural way of linking OR risk factors to OR risk measures for monitoring and managing OR at the business level

5. A BN is a direct representation of the world as seen by the modeler, and not of reasoning processes as in neural networks. The arrows or arcs in the network represent real causal connections, not flow of information during reasoning. This makes them naturaly suited for predictions (Pearl and Russel, 2001)

\subsubsection{Criticisms of Bayesian Networks in Operational Risks Application}

The initial greatest criticism of $\mathrm{BN}$ application in $\mathrm{OR}$ was philosophical in nature and concerns the use of subjective data. However, in OR modelling the use of such data in the form of Control Self Assessment is now generally accepted, which thus weakens this criticism. Indeed, it is hardly possible to avoid subjective assessments in the context of OR.

Some Operational Risk practitioners find BNs fairly complex to establish and maintain. Some critics are of the opinion that the networks demand too much effort and give too little in return; still others regard the issue of obtaining the required numerical probabilities as a major obstacle.

Bayesian networks may appear complex to model, but in reality they are just a structure that represents one's understanding of a process and its dependencies (causes and effects). Representing one's understanding of a process with its dependencies graphically does not seem to be an enormous task. BN application in OR appears difficult to establish and maintain because there is not much guidance in the literature on how OR practioners can implement them. The problem of eliciting numerical probabilities is essentially the same as that encountered in carrying out a Control Self Assessment in Operational Risk.

One can argue however, that the number of probabilities required in an OR BN is larger than what is usually elicited in CSA. This is true in a way, but use of 
methods like sensitivity analysis in focused elicitation and elicitation of parametric distributions are able to reduce the number of probabilities substantially. The theory behind BNs is set forth in the next chapter. 


\section{Chapter 3}

\section{Bayesian Networks}

\subsection{Introduction on Bayesian Networks}

Bayesian networks (BN) (also generally referred to as Probabilistic networks, Causal Probability networks, Probabilistic cause-effect models) help us to model a domain containing uncertainty. Simply put, Bayesian networks are probabilistic networks derived from Bayes theorem, which allows the inference of a future event based on prior evidence. A Bayesian network consists of a graphpical structure, encoding a domain's variables, the qualitative relationships between them, and a quantitative part, encoding probabilities over the variable (Pearl, 1988). A $\mathrm{BN}$ can be extended to include decisions as well as value or utility functions, which describe the preferences of the decision-maker. Such models are known as Influence Diagrams.

One important aspect of BNs is its graphical structure, which enables us to represent the components of complex probabilistic reasoning in an intuitive graphical format, making understanding and communicating easy for the mathematically unsophiscated. Another important aspect is the quantitative part of BNs, which is able to accomodate subjective judgements (expert opinions) as well as probabilities based on objective data. Perhaps the most important part of a BN is that they are direct representations of the world, not of reasoning processes. The arrows in the network represents real causal connections and not the flow of information during reasoning (as in rule based systems and neural networks) (Pearl and Russel, 2001).

Bayesian networks are Directed Acyclic Graphs (DAGs). A graph is defined as a set of nodes (vertices) and a set of edges (arcs) with each arc being a pair of nodes 
(Brauldi, 1992). If the two nodes within each arc $\left(X_{i}, X_{j}\right)$ are ordered, then the arcs have a direction assigned to them. This is called a Directed Graph. A cycle within a graph is a path that starts and ends at the same node. A path is a series of nodes where each successive node is connected to the previous node by an arc and each connecting arc has a directionality going in the same direction as the series of nodes. A DAG is therefore a directed graph that has no cycles.

The relationships in a graph is usually described as it's done in human genealogies. A parent-child relationship is present when there is an $\operatorname{arc}\left(X_{1}, X_{2}\right)$ from $X_{1}$ to $X_{2}$. In Figure 3.1, $X_{1}$ is the parent of $X_{2}$ and $X_{2}$ is the child of $X_{1}$. The parentchild relationship is also extended to an ancestor-descendent relationship. $X_{1}$ is the ancestor of $X_{3}$ and $X_{4}$ and $X_{3}$ and $X_{4}$ are the descendents of $X_{1}$.

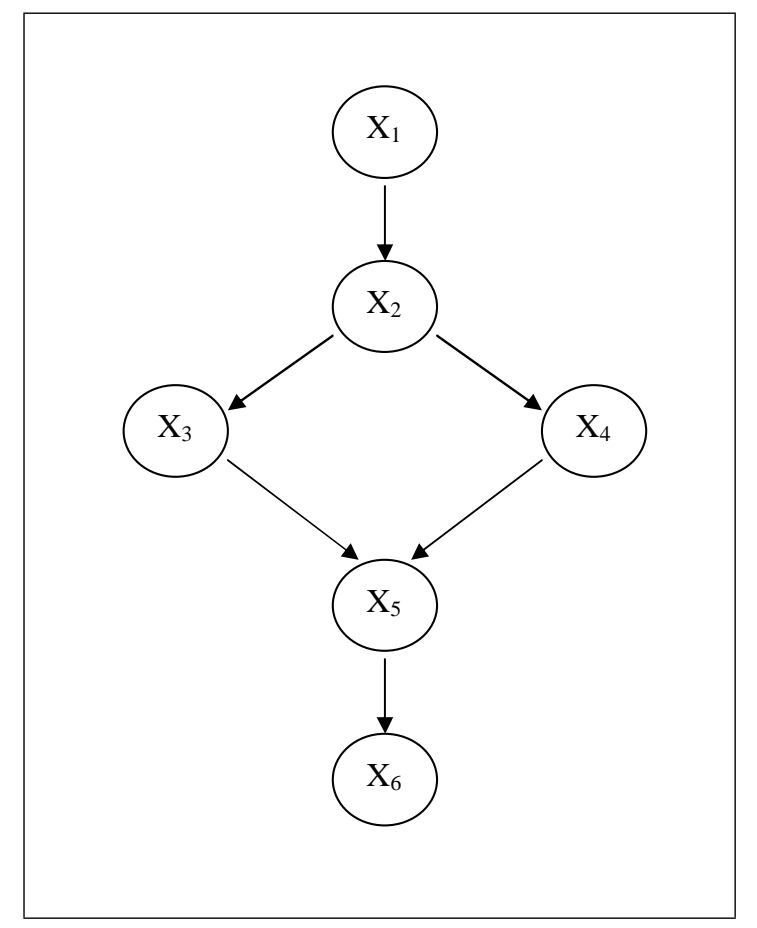

Figure 3.1: An example of a Bayesian network

Each node in a Bayesian network is associated with a set of probability tables. The nodes represent proposition variables of interest and can be either discrete or continuous. The links or arcs in a Baysian network specify the independence assumptions that must hold between the random variables. The network has built-in independent assumptions that are implied in the graphical representation. A causal network according to Pearl (2000), is a Bayesian network with the added property that the parents of each node are its directed causes. 


\subsection{Areas of Application}

Bayesian networks have had considerable applications in many fields both in academia and industry. The major application area in both fields has been diagnosis, which lends itself very naturally to the modelling techniques of Bayesian networks. In the academic field, Nikovski (2000) applied it to problems in medical diagnosis, Hansson and Mayer (1989) in heuristic search, Ames et al. (2003); Marcot et al. (2001) in ecology, Heckermann (1997) in data mining and Breese and Heckerman (1999) in intelligent trouble shooting systems.

Industrial application of Bayesian technology spans several fields including medical and mechanical diagnosis, risk and reliability assessment, and financial risk management. An example of medical diagnosis is the Heart Diseas Program developed by the MIT laboratory for Computer Science and Artificial Intelligence. This program assists physicians in the task of differential therapy in the domain of cardiovascular disorders (Long, 1989). One mechanical diagnostic application is the computer trouble shooting SASCO project by University of Aalborg, Denmark and Hewlett Packard (see Jensen et al., 2000). This system is used in several of Hewlett Packard's printers.

In risk and reliability assessment, Philips Consumer Electronics uses BN technology to predict software defects in its consumer electronics (Fenton et al., 2001). Some examples in financial risk management include the credit risk prediction tool BayesCredit ${ }^{1}$ and the iRisk tool for operational risk prediction (Neil et al., 2005).

\subsection{Theory of Bayesian Networks}

A mathematical definition of BN (Jensen, 2001) comprises

- A set of variables and a set of directed edges between the variables.

- Each variable has a finite set of mutually exclusive states.

- The variables together with the directed edges form a $D A G$.

- To each variable $A$ with parents $B_{1}, \ldots, B_{n}$, there is attached the potential table $P\left(A \mid B_{1}, \ldots, B_{n}\right)$.

\footnotetext{
${ }^{1}$ see http://www.hugin.com/cases, visited 27.06.05
} 
Note that if A has no parents, then the table reduces to unconditional probabilities $\mathrm{P}(\mathrm{A})$.

\subsubsection{The Chain Rule for Bayesian Networks}

For any given complete probabilistic model, the joint probability distribution (the probability of every possible event as defined by the values of all the variables) can be specified. Let $X=\left\{x_{1}, x_{2}, \ldots x_{n}\right\}$. The joint probability distribution can be specified as $P(X)=P\left(x_{1}, \ldots ., x_{n}\right) . P(X)$ however, grows exponentially with the number of variables. Bayesian networks gives a compact representation of $P(X)$ by factoring the joint distributions into local, conditional distributions for each variable given its parents. If we let $p a\left(x_{i}\right)$ denote a set of values for $X_{i}$ 's parents, then the full joint distribution is the product of all

$$
P\left(x_{1}, x_{2}, \ldots x_{n}\right)=\Pi P\left(x_{i} \mid p a\left(x_{i}\right)\right)
$$

For the example given in Figure 3.1, the full joint probability is given as

$$
\begin{gathered}
P\left(x_{1}, x_{2}, x_{3}, x_{4}, x_{5}, x_{6}\right) \\
=P\left(x_{1}\right) P\left(x_{2} \mid x_{1}\right) P\left(x_{3} \mid x_{2}\right) P\left(x_{4} \mid x_{2}\right) P\left(x_{5} \mid x_{3}, x_{4}\right) P\left(x_{6} \mid x_{5}\right)
\end{gathered}
$$

The independence structure is seen in the network as

$$
P\left(x_{5} \mid x_{4}, x_{3}, x_{2}, x_{1}\right)=P\left(x_{5} \mid x_{3}, x_{4}\right)
$$

This means that when the independence assumption is respected in the construction of a Bayesian network, the number of conditional probabilities to be evaluated can be reduced substantially.

\subsubsection{Algorithms for Evaluating Bayesian Networks}

A Bayesian network is basically used for inference; computing the belief (conditional probability) of every node given the evidence that has been observed so far. There are essentially two kinds of inference in Bayesian networks: 1) belief updating and 2) belief revision (Pearl, 1988). In belief updating the posterior probability $P(Y \mid E)$ of query node $Y$ given some observed values of evidence $E$ is computed. In belief revision the most probable instantiation of some hypothesis variables, given the observed evidence, is computed. When the hypothesis 
variables are all non-evidence nodes in belief revision, the computation is known as computing the Most Probable Explanation (MPE). This means there is no other explanation that has a higher probability of occurring than that computed. Inference computation in Bayesian networks is NP-hard (that is, a mathematical way to indicate that it is impossible to compute within reasonable time) (Cooper, 1990; Dagum and Luby, 1993). At the moment, there exist two classes of inference algorithms - one that gives exact solutions and the other that gives approximate solutions (the solution is within some small distance of the correct answer with high probability).

\subsubsection{Exact Inference Algorithms}

The are several classes of exact inference algorithms. These include the polytree algorithm (Pearl, 1988), the clique-tree (Lauritzen and Spiegelhalter, 1988) and junction-tree algorithms (Cowell, 1999). The Clique tree and Junction tree are also known as the clustering algorithms. Others are the arch reversal and node reduction algorithm (Shacter, 1986, 1990), the variable elimination algorithm (Zhang and Poole, 1994), the symbolic probability inference algorithm (Shacter et al., 1990; Li and D'Ambrosio, 1994) and the differential approach algorithm (Darwiche, 2000). A recent survey of these alternative methods is found in Guo and Hsu (2002). Pearl's method is one of the earliest algorithms which has been improved upon. The following simplified description of Pearl's method is taken from Stephenson (2000).

Belief propagation, according to Pearl (1988) proceeds as follows: Let e be the set of values for all the observed variables. For any variable $X$, e can be split into two subsets: $\mathbf{e}_{\mathbf{X}}^{-}$, which represents all of the observed variables that are descendants of $X$ (including $X$ itself if it is observed), and $\mathbf{e}_{\mathbf{X}}^{+}$, which represents all of the other observed variables. The impact of the observed variables on the beliefs of $X$ can then be represented using the following two values:

$$
\begin{aligned}
& \lambda(X)=P\left(\mathbf{e}_{\mathbf{X}}^{-} \mid X\right) \\
& \pi(X)=P\left(X \mid \mathbf{e}_{\mathbf{X}}^{+}\right)
\end{aligned}
$$

$\lambda(X)$ and $\pi(X)$ are vectors. This is because $x$ has multiple values. The elements of $\lambda(X)$ and $\pi(X)$ are associated with each of the discrete values for $X$ :

$$
\lambda(X)=\left[\lambda\left(X=x_{1}\right), \lambda\left(X=x_{2}\right), \ldots, \lambda\left(X=x_{l}\right)\right]
$$




$$
\pi(X)=\left[\pi\left(X=x_{1}\right), \pi\left(X=x_{2}\right), \ldots, \pi\left(X=x_{l}\right)\right]
$$

The following posterior distribution is obtained using (3.1) and (3.2)

$$
P(X \mid \mathbf{e})=\alpha \cdot \lambda(X) \cdot \pi(X)
$$

with $\alpha=\frac{1}{P(\mathbf{e})}$, and with pairwise multiplication of the items in $\lambda(X)$ and $\pi(X)$. To find the new beliefs, equation (3.5) has to be computed. The $\lambda(X)$ and $\pi(X)$ values are then passed between variables in an orderly fashion. $\lambda(X)$ and $\pi(X)$ are computed as follows:

\section{Computing $\lambda$.}

$\lambda(X)$ is computed using $\lambda\left(Y_{1}\right), \lambda\left(Y_{2}\right), \ldots, \lambda\left(Y_{m}\right)$, where $Y_{1}, Y_{2}, \ldots, Y_{m}$ are all children of $X$. First, when $X$ is observed as $x^{0}$, the elements of the vector $\lambda(X)$ are assigned as follows:

$\lambda\left(x_{i}\right)=0$ if $x_{i} \neq x^{0}$

$\lambda\left(x_{i}\right)=1$ if $x_{i}=x^{0}$

For the case where $X$ is not observed, we have $\mathbf{e}_{\mathbf{X}}^{-}=\bigcup_{i=1}^{m} e_{Y_{i}}^{-}$. Using (3.1) $\lambda(X)$ is expanded as:

$$
\begin{aligned}
\lambda(X) & =P\left(\mathbf{e}_{\mathbf{X}}^{-} \mid X\right) \\
& =P\left(\mathbf{e}_{\mathbf{Y}_{1}}^{-}, \mathbf{e}_{\mathbf{Y}_{\mathbf{2}}}^{-}, \ldots, \mathbf{e}_{\mathbf{Y}_{\mathbf{m}}}^{-} \mid X\right) \\
& =P\left(\mathbf{e}_{\mathbf{Y}_{\mathbf{1}}}^{-} \mid x\right) \cdot P\left(\mathbf{e}_{\mathbf{Y}_{\mathbf{2}}}^{-} \mid X\right) \ldots . P\left(\mathbf{e}_{\mathbf{Y}_{\mathbf{m}}}^{-} \mid X\right) \\
& =\lambda_{Y_{1}}(X) \cdot \lambda_{Y_{2}}(X) \ldots \lambda_{Y_{m}}(X),
\end{aligned}
$$

using the fact that $e_{Y_{1}}^{-}, e_{Y_{2}}^{-}, \ldots, e_{Y_{m}}^{-}$are conditionally independent of each other, and defining the following:

$$
\lambda_{Y_{i}}(X)=P\left(\mathbf{e}_{\mathbf{Y}}^{-} \mid X\right)
$$

For each $\lambda_{Y_{i}}(X)$, its computation proceeds as follows: 


$$
\begin{aligned}
\lambda_{Y_{i}}(X) & =P\left(\mathbf{e}_{\mathbf{Y}}^{-} \mid X\right) \\
& =\Sigma_{y_{i}} P\left(\mathbf{e}_{\mathbf{Y}_{\mathbf{i}}}^{-}, y_{i} \mid X\right) \\
& =\Sigma_{y_{i}} P\left(\mathbf{e}_{\mathbf{Y}_{\mathbf{i}}}^{-} \mid y_{i}, X\right) \cdot P\left(y_{i} \mid X\right) \\
& =\Sigma_{y_{i}} P\left(\mathbf{e}_{\mathbf{Y}_{\mathbf{i}}}^{-} \mid y_{i}\right) \cdot P\left(y_{i} \mid X\right) \\
& =\Sigma_{y_{i}} \lambda\left(y_{i}\right) \cdot P\left(y_{i} \mid X\right)
\end{aligned}
$$

The final equation shows that to compute the value $\lambda(X)$, we only need the $\lambda^{\prime} s$ from all of $X$ 's children, as well as the conditional probabilities from all of $X$ 's children. This means that to compute a variable's $\lambda$ 's we need to first compute its children's $\lambda$ 's. In a compact form, the vector $\lambda(X)$ is computed by:

$$
\lambda(X)=\prod_{c \in \text { children }(X)} \Sigma_{v \in c} \lambda(v) . P(v \mid X)
$$

\section{Computing $\pi$.}

$\pi(X)$ is computed using $X$ 's parent $Y$. Now using (3.2):

$$
\begin{aligned}
\pi(X) & =P\left(X \mid \mathbf{e}_{\mathbf{X}}^{+}\right) \\
& =\Sigma_{y_{i}} P\left(X, y_{i} \mid \mathbf{e}_{\mathbf{X}_{\mathbf{i}}}^{+}\right) \\
& =\Sigma_{y_{i}} P\left(X, \mid y_{i}, \mathbf{e}_{\mathbf{X}_{\mathbf{i}}}^{+}\right) \cdot P\left(y_{i} \mid \mathbf{e}_{\mathbf{X}}^{+}\right) \\
& =\Sigma_{y_{i}} P\left(X, \mid y_{i}\right) \cdot P\left(y_{i} \mid \mathbf{e}_{\mathbf{X}}^{+}\right) \\
& =\Sigma_{y_{i}} P\left(X, \mid y_{i}\right) \cdot \pi\left(y_{i}\right)
\end{aligned}
$$

The foregoing shows that to compute the value $\pi(x)$, we need the $\pi$ 's from $X$ 's parents as well as the conditional probabilities from $X$. It also means that to compute a variable's $\pi$, we need to first compute its parent's $\pi$.

There are potential problems in doing inference with Pearl's method due to the cycles when the directionalities are removed (see Pearl, 1988, chap. 4.4). Consequently, the junction-tree algorithm (Cowell, 1999) has become more popular and useful. In this algorithm, a DAG is first transformed into a tree whose nodes are cliques. The $\lambda$ and $\pi$ values are then propagated throughout the junctiontree. Propagation may be done by Pearl's method or any other method. The junction-tree algorithm proceeds as follows: 
1. Moralize the Bayesian network

2. Triangulate the moralized graph

3. Let the cliques of the triangulated graph be the nodes of a tree, which is the desired "junction-tree"

4. Propagate the $\lambda$ and $\pi$ values throughout the junction-tree to do inference. Propagation will produce the posterior probabilities.

From graph theory, moralizing a graph is done by marrying the parents for each node in a DAG. This is achieved by adding an undirected edge between each parent and going furher to remove the directionalities from all the original edges, resulting in an undirected graph. A triangulated graph is an undirected graph where any simple cycle with at least four nodes also has at least one chord. For a given path, a chord is an edge that does not appear in the path which is between two nodes that occur in the path. A clique in a graph is a subset of nodes which is complete and cannot be made larger while still being complete.

\subsubsection{Approximate Inference Algorithms}

Approximate solutions have been developed because of the running time exponential (exponential time limitations) of exact solutions. According to Guo and Hsu (2002) approximate inference algorithms can be grouped into stochastic simulation algorithms, model simplification methods, search based methods, and loopy propagation methods. The most well-known of these methods are the stochastic simulation algorithms, which can be subdivided into importance sampling algorithms, and Markov Chain Monte Carlo (MCMC) methods. What these algorithms do is to randomly posit values for some of the nodes and use them to pick values for the other nodes. The main difference between the importance sampling methods and MCMC methods is that samples used in the former are independent whereas those used in the later are dependent.

Logic sampling (Henrion, 1989) is the simplest of the importance sampling methods. A simple illustration of this approach by Charniak (1991) proceeds as follows:

1. Guess the root nodes in accordance with the prior probabilities. For example, if $v$ is a node, and $P(v)=0.2$, one randomly chooses a value for this node but in such a way that it is true about $20 \%$ of the time. 
2. Work your way down the network, guessing the value of the next lower node on the basis of the values of the higher nodes. Thus if say, the nodes $a$ and $b$, which are above $c$, have been assigned true and false respectively, and $P(c \mid \neg b)=0.8$, then we pick a random number between 0 and 1 , and if it is less than 0.8 , we assign $c$ to true, otherwise, false.

3. Continue the procedure all the way down the network and track how often each of the nodes is assigned to each of its values.

This simple procedure works well when no evidence has been observed. When evidence is observed most samples generated will be inconsistent and wasted. Various improvements to this simple procedure includes the likelihood weighting method (Fung and Chang, 1989; Shacter and Peot, 1990) the backward sampling method (Fung and Favero, 1994), the self and heuristic importance method(Shacter and Peot, 1990), and the adaptive importance sampling method (Cheng and Druzdzel, 2000).

The MCMC methods work well when there are no extreme probabilities in the Conditional Probabilities Tables. These methods rely on Gibbs sampling, Metropolis sampling and Hybrid Monte Carlo sampling. A good overview of the other approximate methods; model simplification methods, search based methods and loopy belief propagation can be found in (Guo and Hsu, 2002).

\subsection{Realization of Bayesian Network}

Galan Caballero (2005) provides a simple flow diagram (Figure 3.2) for realizing a BN. The first step is to define the domain problem that specifies the purpose of the BN. This is followed by identifying the variables or nodes that are important for the domain problem. Next, the relationships among the variables or nodes are identified and represented in a graphical structure. The model structure obtained is then validated with the experts. If there is no agreement among the experts on the model structure, the process goes back to the previous step until an agreement is reached. The last three steps include eliciting expert opinion (also refered to as quantifying the network), running plausible scenarios with the network (network application), and fine tuning the estimates over time (maintaining the network).

Building a Bayesian network is an iterative process and also a trade off between a very detailed and rich model to obtain "accurate" results on the one hand, and the cost and complexity of obtaining the detailed probabilities and maintaining the network afterwards on the other hand. 


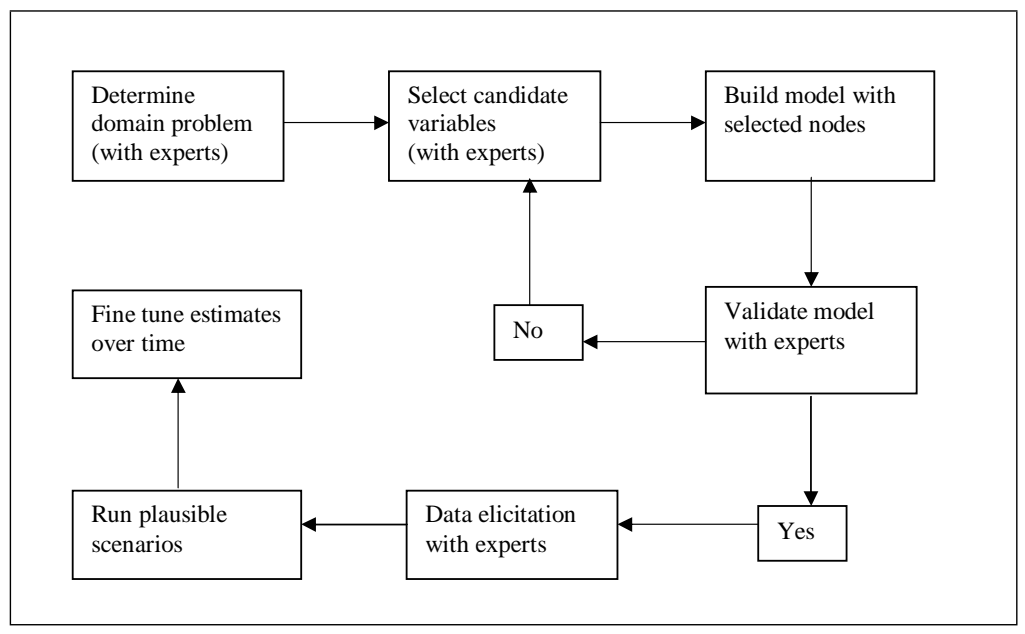

Figure 3.2: A BN flow diagram

\subsubsection{Nodes, Network and Structural Validation}

The nodes or variables in a Bayesian network are usually of three types: 1) hypothesis variables - variables of interest (target variables), 2) information variables - variables whose state can be observed, and 3) mediating variables - variables introduced for a special purpose, for example, to either reflect the independence properties in the domain or to facilitate the aquisition of conditional probabilities. The various nodes are connected by arrows or arcs into a network that reflects the domain problem. The network also shows the various relationships among the variables. There is no unique network for a given domain problem since the network is a representation of the understanding of the modeler and experts of the process being modeled.

The definition of Bayesian networks and the chain rule for Bayesian networks given earlier requires that the independence assumptions are respected. Theoretically, there are two ways of checking that these assumptions hold. The first method, known as d-separation, is due to Pearl (1988). The second, based on set theory, is due to Lauritzen et al. (1990).

The rules of d-separation are based on the three fundamental connections in Bayesian networks;

1. Serial Connection $X \longrightarrow Y \longrightarrow Z$ : Information may be transmitted through the connection unless the state of $\mathrm{Y}$ is known. Example: If we observe the 
rain falling $(Y)$, any knowledge that there is a dark cloud $(X)$ is irrelevant to any hypothesis (or belief) that the lawn is wet $(Z)$. On the other hand, if we do not know whether it rains or not, observing a dark cloud will increase our belief about rain, which in turn will increase our belief about the lawn being wet.

2. Diverging Connection $X \longleftarrow Y \longrightarrow Z$ : Information may be transmitted through the connection unless the state of $Y$ is known. Example: If we observe the rain falling $(Y)$ and then that the lawn is wet $(X)$, the added knowledge that the lawn is wet $(X)$ will tell us nothing more about the type of weather report to expect from the radio $(Z)$ than the information gained from observing the rain alone. On the other hand, if we do not know whether it rains or not, a rain report in the radio will increase our belief that it is raining, which in turn will increase our belief about the lawn being wet.

3. Converging Connection $X \longrightarrow Y \longleftarrow Z$ : Information may be transmitted through the connection only if information about the state of $\mathrm{Y}$ or one of its descendants is available. Example: If we know the lawn is wet $(Y)$ and that the sprinkler is on $(X)$, then this will effect our belief about whether it has been raining or not $(Z)$, as the wet lawn leads us to believe that this was wet by the sprinkler, rather than the rain. On the other hand, if we have no knowledge about the state of the lawn, then observing that it rains will not affect our belief about whether the sprinkler has been on or not.

Two variables $X$ and $Z$ are d-separated if for all paths between $X$ and $Z$ there is an intermediate variable $Y$ such that either

- the connection is serial or diverging and $Y$ is instantiated (i.e., its value is known), or

- the connection is converging and neither $Y$ nor any of its descendants have received evidence.

If $X$ and $Z$ are not d-separated, they are d-connected. Dependence and independence depends on what you know (and do not know). In other words, available evidence plays a significant role when determining the dependence and independence relations.

The second equivalent criteria due to Lauritzen et al. (1990) is described below. Let $A, B$, and $C$ be disjoint sets of variables. Then 
- identify the smallest sub-graph that contains $A, B, C$ and their ancestors,

- add undirected links between nodes having a common child, and

- drop directions on all directed links.

Now, if every path from a variable in $A$ to a variable in $B$ contains a variable in $C$, then $A$ is conditionally independent of $B$ given $C$.

\subsection{Quantifying the Network}

Quantifying the network basically means assessing the probability distributions for each of the nodes represented in the network. The information for assessing the probability distributions can either be obtained from objective past data, if available, or elicitation of probabilities from domain experts. Both types of data can be used separately or in combination with each other.

Objective data are often not available for use in most Bayesian networks. Although some information can be obtained from historical data bases most of these are either insufficient for analysis or not in a form that can be easily encoded into a Bayesian network. An example is the fact that most of the probabilities to be estimated in a BN are conditional probabilities. The difficulties with objective data thus make the use of subjective data more attractive but elicitation of subjective information has its own unique problems.

\subsubsection{Probability Elicitation}

Expert judgement can be used to provide estimates on new, rare, complex or otherwise poorly understood phenomena, or to forecast future events. The process of gathering and encoding expert judgement, or knowledge, into probability or uncertainty distributions through special methods of verbal or written communication is what is known as elicitation of expert opinion, or probability elicitation.

In constructing a Bayesian network, it is a well known fact that elicitation of probabilities is the most difficult aspect of the entire process. There are, however, some means of making the task easier. One such method is to make changes to the graphical structure of the network. This is based on the principle of divorcing parents by introducing intermediate variables (Olesen et al., 1989) or the removal of arcs representing weak dependencies (Kjaerulff, 1994; van Engelen, 1997). Divorcing parents becomes useful when we have a list of variables say, 
$A_{1}, \ldots A_{4}$ all of which are causes of $B$. To specify the distribution $P\left(B \mid A_{1}, \ldots, A_{4}\right)$ would be a difficult task for an expert or would require a large set of cases if the data were extracted from a database. The parents of $\mathrm{B}, A_{1}, \ldots A_{4}$ can be divorced by introducing an intermediary variable, $C$ such that $C$ becomes a child of $A_{1}, A_{2}$ and a parent of $B$ with $A_{3}, A_{4}$ also being parents of $B$.

Another method is based on the use of parametric probability distributions with the aim of reducing the probabilities that have to be assessed directly as well as providing some rules for the computation of the other required probabilities (Heckermann and Breese, 1996; Henrion, 1989; Pearl, 1988). Specifically, instead of eliciting point probabilities estimates, we rather elicit probability distributions over the states of nodes from the experts. This is quite straightforward if we have some prior knowledge, or if the nature of quantities to be assessed suggest they follow a particular class of probability distributions.

The method of focused elicitation based on sensitivity analysis (van der Gaag et al., 1999) is an approach of shortening the time spent by experts in providing

probability estimates. Sensitivity analysis in general is a method to investigate the effects of systematically varying a model's parameters on its predictions. When applied to Bayesian networks, it focuses probability elicitation on the most influential nodes of a network structure. It starts with using the elicitation of crude probabilities to quantify the network, and then use sensitivity analysis to uncover the most influential as well as the uninfluential or redundant nodes within the network. The influential probabilities are then refined using some of the conventional elicitation methods. The procedure is done iteratively, each time refining the probabilities until satisfactory results are obtained. This method can also be used to refine the structure of the network by iteratively deleting uninfluential nodes.

\subsubsection{Protocols and Bias}

Eliciting probabilities from experts is a rather difficult task to carry out. It involves both statistical and psychological issues. This was pointed out by de Fenetti (1969) when he stated that

....the true subjective probability problem consists in the investigations concerning the ways in which probabilities are assessed by more or less educated people, and the way in which such abilities may be improved. This seems to me the field in which the cooperation between all specialists concerned is most wanted, and that is particularly true 
for the expected contributions from psychologists.

- B. deFinetti

Fortunately, over the years some protocols that incorporate psychological theory have evolved. Although there are no universally accepted protocols for this task, there are a few that are accepted by most analysts. The three most common ones are Standford/SRI protocol, the Morgan and Henrion's protocols and the Wallsten/EPA protocol (Morgan and Henrion, 1990).

The Stanford/SRI and Morgan and Henrion's protocol include 5 phases:

1. motivating the expert with the aims of the elicitation process,

2. structuring the uncertain quantities in an unambiguous way,

3. conditioning the expert judgement to avoid cognitive bias,

4. encoding the prob distributions, and

5. verifying the consistency of the elicited distributions.

The Wallsten/EPA protocol includes:

1. preparation of documents that outline the objective of the process

2. description of cognitive heuristics, biases and other relevant issues. The expert reads the document before the elicitation process.

Cooke and Goossens (2000) also developed a guide for elicitation of expert judgement that has a three part structure: preparation of elicitation, elicitation and, post-elicitation.

For every elicitation process there are certain pitfalls or biases that one has to be aware of, and to counter. Bias is an aspect of how people solve problems. It is a deviation from reality, or what we consider desirable, and can degrade the quality of the data, contributing to the problem of garbage-in-garbage-out (Meyer and Booker, 2001). The scientific literature contains two views of bias, with their distinctions based on the reference point from which they are judged. The reference point for one is the concept of objective criteria for reality (cognitive bias) and the other is what the expert knows or does (motivational bias).

Motivational bias is driven by our human needs, like social pressure, management impression and wishful thinking among others. Cognitive bias is due to the way 
human process information and occurs when experts' estimates fail to follow normative statistical or logical rules. Some signs of cognitive bias and other pitfalls are 1) availability bias - experts link their estimates to the frequency with which they can recall an event (Tversky and Kahneman, 1974), 2) adjustment and anchoring - judgements are anchored at a starting value and adjusted outwards usually insufficiently (Winkler, 1967; Alpert and Raiffa, 1982), 3) inconsistency - occurs when experts become inconsistent in their reasoning during the elicitation process, 4) overconfidence - difficulty in assessing the tails of a distribution (Wallsten and Budescu, 1983), and 5) handsight bias - if the expert has seen the sample data, the elicited opinion may have already been updated on the basis of the data (Morgan and Henrion, 1990). Methods of dealing with bias are treated in Chapter 5.

\subsubsection{Validation of Estimates}

Expert opinion belongs to the domain of inductive probability. The statistics literature distinguishes between statistical, or objective probability, and inductive or subjective probability (Carnap, 1950; Bulmer, 1979). An objective probability is a statistical probability that is grounded in a concrete reality and can be verified by repeated trials. Statistical probabilities or relative frequencies, are usually associated with some ex-post calculation and or a complete knowledge of a datagenerating process; and may therefore have little to do with fundamental forms of uncertainty emerging from incomplete knowledge.

Inductive probability, on the other hand, represents the degree of belief in an uncertain and unrepeatable event. It acts as a guide to life and is formed even when an anticipated event is unprecedented. Subjective probability mostly deals with ex-ante predictions; they are formed in the face of uncertainty and incomplete knowledge. Inductive probability has found greater importance in some applications than statistical probability because either knowledge of an underlying reality is limited, or a particular anticipated event is unprecedented.

Validations of inductive probability becomes difficult because of its belief-based nature. Two common elements that are used to validate expert opinion are coherency (Lindley et al., 1979) and reliability (Wallsten and Budescu, 1983). Kandane and Wofson (1998) are of the opinion that any attempts at validation should only use other statements of belief by that person as data.

The validation problem can also be approached differently. One can assess the effectiveness of an elicitation process prior to conducting the real elicitation and 
thus would not have to validate the estimates afterwards. This approach is based on the assumption that the degree of closeness of the elicited estimates to what is in the expert's head, is dependent on the effectiveness of the elicitation scheme. With this approach the effectiveness of various elicitation schemes must be evaluated. Comparisons of elicitation schemes in the literature follow three lines of reasoning: 1) expert's preference, 2) benchmark mode, and 3) performance measure. The expert's preference method is based on the assumption that an elicitation method preferred by an expert will yield better quality estimates. The benchmark model (see Lichtenstein et al., 1978; Price, 1998) compares the results of elicitation using various schemes against an existing benchmark (gold standard) model of a domain (or a correct answer that is assumed to be widely known). The last approach, performance measure (see Lichtenstein et al., 1982; van der Gaag et al., 1999) compares the predictive performance of models derived using various methods.

\subsection{Strengths and Limitations of Bayesian Net- works}

Some strengths or advantages of BNs are as follows:

1. The graphical nature of $\mathrm{BN}$ makes it a powerful communication tool. The relationships (causal) among the variables or nodes can be seen easily without the need to compute probabilities.

2. Ability to combine subjective data (expert knowledge) and objective data. This is a great advantage especially when objective data is scarce or when there is the need to incorporate expert opinion in the model

3. BNs are capable of modeling highly complex systems. The areas of application mentioned earlier attest to that.

4. The network or model can be easily updated, or modified in the light of new information such as poor performance.

5. BNs can be used to perform sensitivity or "what-if" analyses to examine the sensitivity of predictions, or conclusions against initial assumptions.

6. BNs are based on efficient evidence propagation algorithms that dynamically update the model given all current data. 
7. Lastly, BNs contain algorithms that handle missing observations in an exact way, since basic axioms of probability are used to compute the probabilities.

Bayesian networks do have some limitations. One of such limitations is the fact that a BN model is only as good as the modeler and experts who produced it, since it is a representation of the modeler and the experts' perceptions of reality. There is therefore no wrong or correct model, but a best fit given the modeler and experts.

Another limitation centers on the extent of the quality of the prior beliefs used in Bayesian inference processing. The usefulness of a BN is based on the reliability of its prior knowledge. An excessively optimistic or pessimistic expectation of the quality of these prior beliefs will either distort the entire network or invalidate the results. Related to this concern is the selection of the statistical distributions induced in modeling the data. Selecting the proper distribution model to describe the data has a notable effect on the quality of the resulting network.

\subsubsection{Concluding Remarks}

Bayesian belief networks have established themselves as a valuable and useful tool for reasoning under uncertainty. The limitations of BNs can be addressed through careful modeling. The ability of BNs to perform scenario or "what- if" analyses puts it far above other operational risk causal models. Scenario analysis is at the core of operational risk management especially at the tails of distribution where data is always sparse. BNs are therefore a natural choice for operational risk management.

An Operational Risk Bayesian model will enable risk or business managers to manage the day-to-day operational risk and measure the required economic capital at the same time. As a tool, it is possible to predict what might happen (severity) if something goes wrong, so that efforts can be concentrated to prevent or to reduce the chances of repeated failures. 



\section{Chapter 4}

\section{Introduction to the Case Study}

\subsection{Introduction}

This part of the work (Part II) is a case study that outlines the issues involved in implementing a Bayesian network (BN)for the Foreign Exchange (FX) and Money Market (MM) settlement department in a bank. It shows how a BN for managing operational risk in FX settlement is implemented. The entire section is composed of three chapters. This Chapter, introduces the problem, sets out the specific objectives, the scope of the modeling and finally some decisions made before the start of the modeling exercise. Chapter five then deals with how the BN was built and quantified. Chapter seven is on model application - model results, model validation, and lastly, how the model can be used to manage operational risk in Foreign Exchange and Money Market settlement.

\subsection{Problem Description}

The literature on operaional risk contains some writings on the advantages of managing operational risk with Bayesian belief networks (see Alexander, 2003; King, 2001; Ramamurthy et al., 2005). Some few illustrative but simplistic examples on managing Operational Risk with BNs are also given. There is, however, insufficient documentation giving a detailed procedural guide on how a risk or business unit manager can implement a BN to model a desired process.

The main problem that a risk or business manager faces here are how to go about implementing a BN in general, how to model the network structure and quantify the network. Should subjective or objective data be used? If I subjective data 
is used, how should one go about the elicitation process? What are the biases involved in the elicitation process and how can one counter them? What are the statistical issues involved in implementing a $\mathrm{BN}$ and how can one validate the model? These are some of the issues that the case study seeks to address.

\subsection{Specific Objectives in this Case Study}

The primary objective of our case study is to develop procedures for implementing a Bayesian belief network for the management of operational risk in FX and MM settlement. Secondary to this is to apply the Bayesian network to a real-world situation and demonstrate its capabilities in managing operational risk within the FX and MM settlement process.

The first step in the construction of a BN is to establish the objective or purpose of the network. An Operational risk Bayesian network can be used for several purposes. These may include the use of the network to 1) manage operational risk at the business line level or, 2) calculate economic or regulatory capital charge and 3) a combination of the above. Most Operational risk practitioners are of the view that operational risk Bayesian networks should either be used for managing operational risk, or used for quantifying operational risk internally (not for regulatory purposes). This view is shared by the author. Thus the main objective of the BN developed in this work is to assist in managing operational risk and also to compute economic capital, but solely for internal management purposes.

\subsection{Scope of Modeling and Decisions}

Foreign exchange and money market settlement process includes the following sub-process: 1)pre-trade preparation, 2) trade capture, 3) confirmation, 4) netting, 5) settlement and 6) nostro reconciliation. The case study covers only four of these sub-processes being; trade capture, confirmation, netting and settlement. Within these sub-processes, further emphasis is placed heavily on confirmation and settlement because that was the sub-processes of primary interest to the bank in question.

Some decisions were made prior to the modeling of the FX and MM settlement process. These decisions have to do with the use of either subjective or objective data and the choice of Bayesian modeling shell for realizing the networks. 
Bayesian networks can be quantified with either subjective or objective data or both. Objective data is usually used if available and can easily be encoded into the network. Our modeling domain does indeed have some objective data available. However, the network was developed with subjective data (expert opinion) and the available objective data (historical loss data) was used only as a means of "validating" the developed model. The statistical analysis of the work was done using the "R" statistical software package.

There are several software packages for realizing a Bayesian network. The Hugin Lite free trial version ${ }^{1}$ was chosen as the modeling shell for this work. This shell is very user friendly, which is a good thing for business line managers with no specific expertise in Bayesian networks. This trial version, which is free, handles a maximum of 100 states. It has an expression builder for specifying known distributions and also allows the use of continuous nodes.

\footnotetext{
${ }^{1}$ see www.Hugin.com
} 



\section{Chapter 5}

\section{Construction of a Bayesian Network for Foreign Exchange Settlement}

\subsection{The Foreign Exchange and Money Market Settlement Process}

After Foreign Exchange (FX)and Money Market(MM) transactions are made, there is the necessity for clearing and settling the transactions. Clearing and settlement are known as back office operations. Clearing refers to processing a trade and establishing what the parties to the trade owe each other. Settlement refers to the transfer of value between the parties so the trade is completed (Group of Thirty, 1989).

There are several steps involved in the clearance and settlement process. The first is to transmit the trade instructions from the traders (front office) to the back office. This is usually done straight through. The second is comparing and matching of trade details between the buyer and the seller to ensure that both agree on what is to be traded and on the terms. The third step is the transmission of trade details to the payment systems which then generates payment transfer notes for final payment into the designated accounts. In case of money market trades confirmations are normally not used. If the bank takes money, the counterparty normally transfers the money within two days. The payment itself is the best confirmation the bank can receive. Figure 5.1 shows a schmatic presentation of the entire FX and MM trade process. The thesis concentrates 
more on the back office pre- and actual settlement, and the payment processing. Since the other activities of trade capture in the front office and reconciliation do have an effect on these two, they are also included in the modeling.

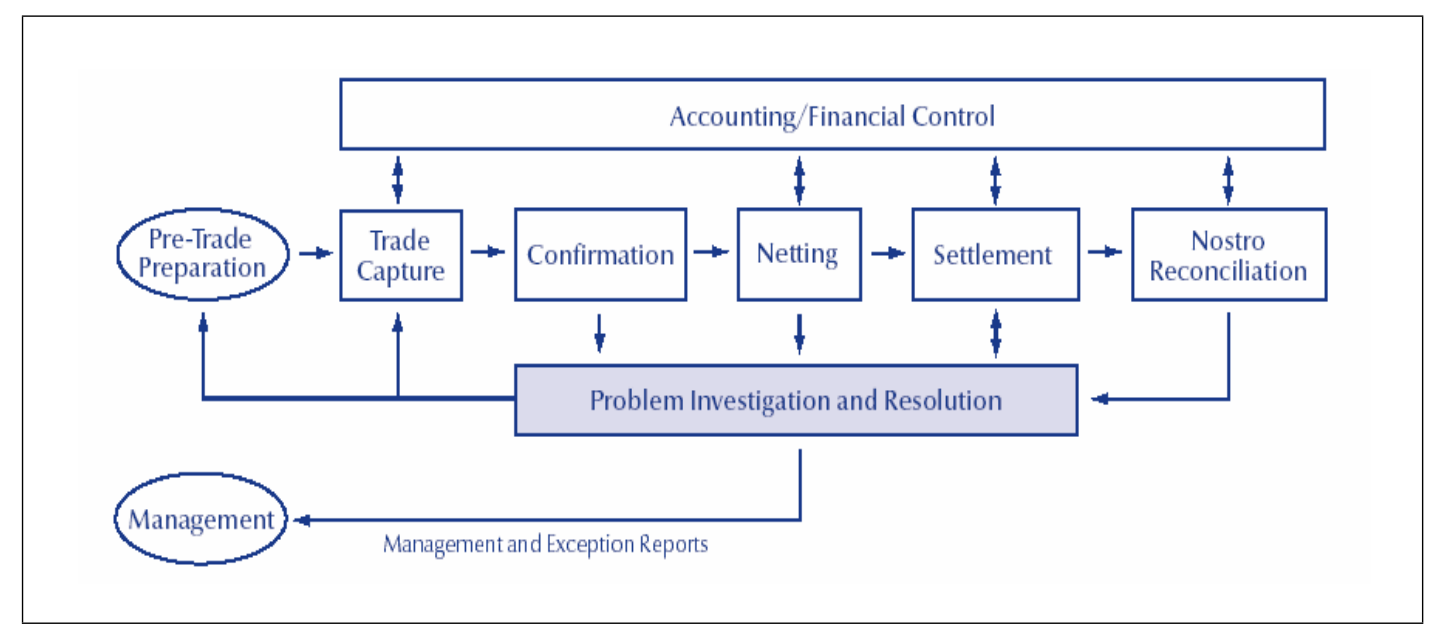

Figure 5.1: FX process flow. Source: The Foreign Exchange Committee, Federal Reserve Bank of New York

\subsection{Building the Network}

Constructing the BNs used for the FX and MM settlement processes comprised two stages: 1) obtaining the network structure, which can also be termed as structural model building and 2) quantifying the obtained structure. The processes involved in the two stages follow the flow diagram (Figure 3.2) given in Chapter 3. It will be recalled that the flow diagram shows an iterative process with components for the structural model building part such as establishing the domain problem (already covered in Chapter 4), selecting candidate variables (node identification), building the model structure, and validating the structure. The components of the quantitative part includes data elicitation and running of plausible scenarios. 


\subsection{Structural Model Building}

The processes involved in obtaining the structure of the BN for FX and MM settlement process starts with what is termed risk and process mapping. This is followed by node identification and ends with putting them all together and detailing out the dependencies to obtain the network structure.

\subsubsection{Risk, Process Mapping and Nodes Identification}

The purpose of risk and process mapping is to identify the risk drivers or causal factors in the entire FX and MM settlement process. Risk drivers or causal factors are measures that drive the inherent risk profile of the organisation. Changes in these measures would usually indicate that the risk profile is changing. Examples of risk drivers include transaction volumes and level of automation. Risk drivers are more forward-looking and therefore predictive of future issues (Haubenstock, 2003). In operational risk, risk drivers and causal factors are simply the underlying reasons giving rise to an OR event. The terms risk factors, risk drivers and causal factors are usually used interchangeably.

The process of mapping and identification of risk factors is done with the help of the business managers and the operational risk experts responsible for the particular business line. This exercise helps the modeler to get a deeper understanding of the settlement process in place. In addition, business managers may be confronted for the first time with a detailed analysis of the settlement process and the drivers of operational risk.

To identify the risk drivers or causal factors, the entire value chain is broken down into sub-processes: 1) Trade capture in the front office 2) Processing in the back office 3) Payment and settlement, and 4) Accounting and nostro reconciliation. For each of these sub-processes the activities involved and what these particular activities depend on are outlined. The possible risk that could occur within the various activities and the factors that drive these risks are also identified. For example, the activities involved in trade capture are: make trade, book trade and transmit trade instructions. These activities depend on the front office system, and the employees (personnel) at the front office. The possible risks are erroneous booking, system transmission failure or error and untimely trade entry. The risk drivers are trade volume, availability of supporting system and the level of employee skills. A complete coverage of the entire value adding process is given in Table 5.1. 
The Risk and process mapping produces a list of activities, dependencies, possible risks, causal factors, and KRIs which are all candidates for use as nodes in the construction of the BN. From this list the important variables, which represent the KRIs, causal factors and internal control environment that are deemed relevant for our domain problem are selected for use in the Bayesian network. Some of the variables that describe the same thing are lumped together and given a common name. It is these variables, now called nodes, that are connected together to obtain the network structure.

\begin{tabular}{|c|c|c|c|c|}
\hline & $\begin{array}{l}\text { Trade capture in } \\
\text { Front Office }\end{array}$ & $\begin{array}{l}\text { Back Office } \\
\text { processing }\end{array}$ & $\begin{array}{l}\text { Payment / settlement } \\
\text { process }\end{array}$ & $\begin{array}{l}\text { Acct / nostro } \\
\text { recociliation }\end{array}$ \\
\hline Activities & $\begin{array}{l}\text { make trade } \\
\text { book trade } \\
\text { transmit trade instruct. }\end{array}$ & $\begin{array}{l}\text { receive trade instruct. } \\
\text { validate trade } \\
\text { matching } \\
\text { confirm with } \\
\text { counterparty } \\
\text { transmit trade }\end{array}$ & $\begin{array}{l}\text { generate transfer notes } \\
\text { transmit to } \\
\text { German Bundesbank } \\
\text { Nostro agent for exec } \\
\text { disposition-nostro acct } \\
\text { mgt }\end{array}$ & $\begin{array}{l}\text { prediction of cash } \\
\text { movements } \\
\text { recieve settled info from } \\
\text { nostro agent } \\
\text { reconcile/compare } \\
\text { expected cash- } \\
\text { movements with actual }\end{array}$ \\
\hline Dependency & $\begin{array}{l}\text { Front office system } \\
\text { Human } \\
- \\
- \\
- \\
-\end{array}$ & $\begin{array}{l}\text { Back office system } \\
\text { System ECART } \\
\text { System GAB } \\
\text { (manual handling) } \\
\text { Human } \\
\text { - }\end{array}$ & $\begin{array}{l}\text { Paymentsystem } \\
\text { Nostro Agent } \\
\text { (system) } \\
\text { RTGS systems } \\
\text { Human }\end{array}$ & \\
\hline Possible risk & $\begin{array}{l}\text { erroneous booking } \\
\text { syst transmission } \\
\text { failure } \\
\text { untimely trade entry } \\
\text { - } \\
\text { - }\end{array}$ & $\begin{array}{l}\text { validation error } \\
\text { confirmation error } \\
\text { - } \\
\text { - } \\
\text { - }\end{array}$ & $\begin{array}{l}\text { payment delays } \\
\text { duplicate payment } \\
\text { misdirected settlement } \\
\text { wrong payments(buy/sell) } \\
\text { none payment }\end{array}$ & $\begin{array}{l}\text { reconciliation errors } \\
\text { untimely acct } \\
\text { reconciliation } \\
\text { (leads to underfunding } \\
\text { and overdraft to cash } \\
\text { acc) } \\
\text { oversight }\end{array}$ \\
\hline $\begin{array}{l}\text { Risk/Causal } \\
\text { factors }\end{array}$ & $\begin{array}{l}\text { trade volume } \\
\text { system availability } \\
- \\
- \\
- \\
- \\
- \\
\text { - } \\
\text { - }\end{array}$ & $\begin{array}{l}\text { validation/confirm } \\
\text { method } \\
\text { (manual/automated) } \\
\text { system availability } \\
\text { trade type } \\
\text { forward/same day } \\
\text { NEWT, AMND, CANC } \\
\text { - } \\
\text { - } \\
\text { - }\end{array}$ & $\begin{array}{l}\text { inaccurate/incorrect } \\
\text { instruct } \\
\text { validation errors } \\
\text { confirmation errors } \\
\text { (settling before } \\
\text { confirmat) } \\
\text { system downtime } \\
\text { currency (euro/others) } \\
\text { foward/same day trade } \\
\text { untimely trade entry(cut } \\
\text { off) } \\
\text { gross/netting settlement }\end{array}$ & $\begin{array}{l}\text { reconciliation method } \\
\text { (automated/manual) } \\
\text { system downtime } \\
\text { external errors (nostro } \\
\text { bank) }\end{array}$ \\
\hline
\end{tabular}

Table 5.1: Risk and process mapping 


\subsubsection{Network Structure}

The selected nodes are connected with arrows to form a structure that shows the dependencies or causal relationships among them. There are basically two methods of doing this: 1) through Bayes learning, where the network structure is learnt from the data if available and 2) manually connecting the nodes with arrows. Giudici (2004) used the former approach in modeling operational risk. The latter method, which is used in this study is preferred since it shows the analyst's understanding of the process. Typical questions asked at this stage are what could cause this, and how is this cause manifested? With answers to these questions the causal relationships are expressed in graphical terms by the arrows linking the various variables.

Building the network structure is an iterative process, which is carried out with the business managers. The process starts with a simple network, which is iterated by the addition and deletion of nodes continuously until an acceptable network is obtained. The network is a trade-off between detail and feasibility. A very detailed network will require several probabilities for quantification and maintenance, making it practically expensive. Parents are divorced, where necessary, to make subsequent elicitation of conditional probabilities less difficult. Since the number of probabilities increase exponentially with the number of parents, the network is kept as simple as possible. The structure of the network represents a modeler's understanding and knowledge of the problem domain and may differ from one modeler to the other, even though they might be working on the same domain problem. That is to say there is no unique solution to the problem but a best fit given the experts, and it is only as good as the experts who produced it.

The settlement process network consists of two separate networks modeling the frequency of failure and the loss given failure (severity). The decision to separate the networks is due to ease of construction and capacity constraints of the modeling shell (evaluation version) being used. Additionally, it was found proper to model the frequency of failure and severity separately and later aggregate through Monte-Carlo simulation methods.

\section{Frequency}

The overall structure of the frequency network shown in Figure 5.2 follows the essential elements of FX settlement as detailed in the risk and process mapping. The structure places a heavy emphasis on back office processing since that is the domain of interest to settlement department. Account reconciliation is excluded 
from the model and trade capture is included for the sake of completeness. The underlying idea behind the network is that front office makes the trades and back office checks and confirms the trades before settlement. If the front office makes an error, it is the responsibility of the back office to detect the errors before the trades are settled. The IT systems, on which the entire process depends, are also included in the network. The network is modeled on a time span of one week.

This network developed can also be used for MM settlement with a few modifications. For example, deleting the node confirmation matching (confirmation is normally not done in MM settlement) and changing the states of the node trade type to the appropriate names, since these are different for money market trades.

The frequency network has a total of 14 chance nodes; three belong to trade capture, seven to back office processing and four to transmission for settlement. The node labeled fail-settlement, showing the number of failed settlement is the hypothesis variable - with a state that is of interest. It represents the KRI that we want to monitor. The mediating variable - variable introduced for a special purpose specifically (in our case) to reflect the acquisition of conditional probabilities is the node labeled vulnerability-to-failure. All the other nodes in the network are information variables - variables with a state that can be observed. Some information variables can however, serve as hypothesis variables, for the purpose of managing operational risk. These are the nodes trade capture, confirmationmatching, and payment-system-input-method.

Decision and utility nodes are not included in the network since, without them, our network is still able to meet our intended objective. The network indicates, for example, that the causal factors for settlement failure are the quality of settlement instructions in place, the method of transmission to the payment system and vulnerability to failure (caused by confirmation matching and trade capture). The nodes in the frequency network requires a total of 615 probabilities for encoding. A description of the individual nodes is given in Table 5.2. 


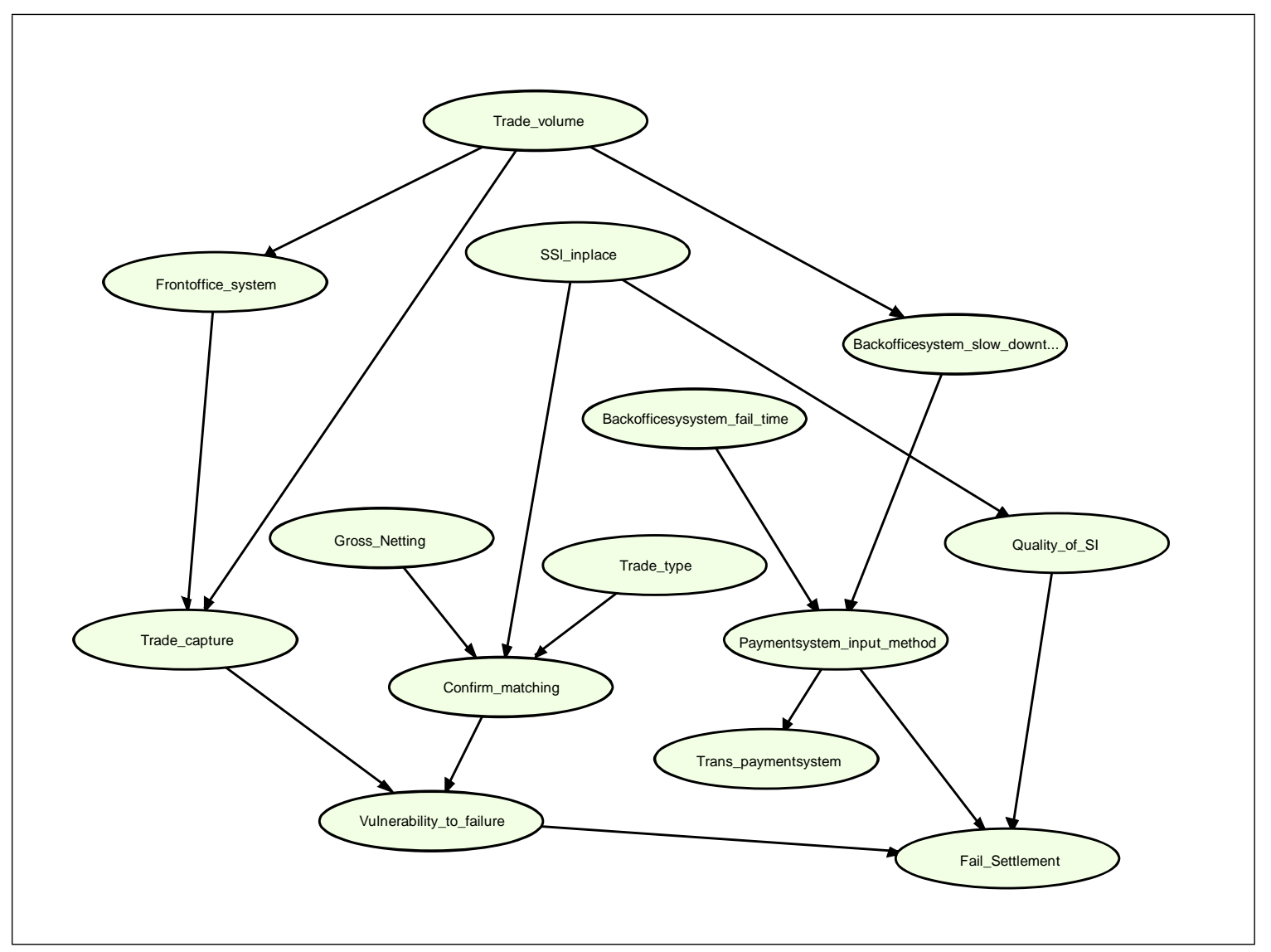

Figure 5.2: Frequency of failure network

\section{Severity}

The severity network shown in Figure 5.3 comprises four chance nodes but requires about 10,500 probabilities for encoding. The loss severity node is the terminal node. This is the hypothesis variable. All other nodes: trade value, days delay, and interest rate regime in the network are information variables. The severity of losses depends on the trade value, the number of days delay in correcting a settlement failure and the applicable interest rate (interest rate regime). A detailed description of each node is given in Table 5.3. 


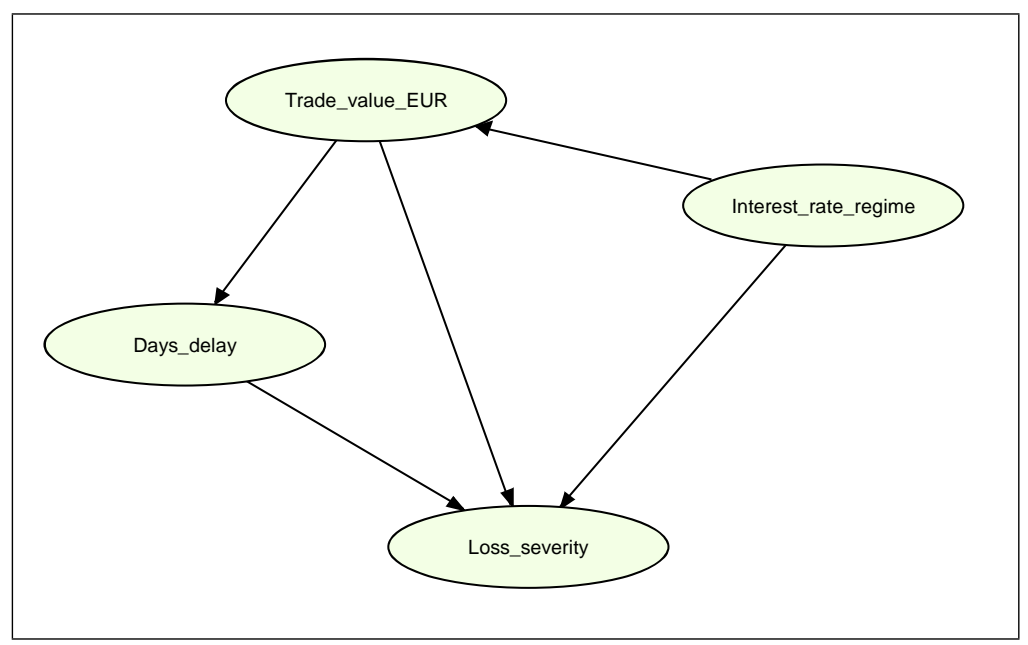

Figure 5.3: Loss severity network

\subsubsection{Conditional Probability and Node Description Ta- bles}

A node in the network represents either a discrete random variable with a finite number of states or a continuous random variable. If the node is discrete, it contains a probability distribution over the states of the variable that it represents. If the node is continuous, it contains a density function (usually specified through the mean and variance parameters) for the random variable it represents. For example, the node trade volume in the frequency network is discrete with states less than 40000 trades, between 40000 to 60000 trades, and more than 60000 trades.

The choice between continuous and discrete nodes is the prerogative of the modeler. Moreover, most Bayesian network modeling shells available do not admit continuous nodes. Those that do, for example, the Hugins modeling shell, only allow the specification of Gaussian density functions. The majority of the nodes in the frequency and severity networks follow distributions other than Gaussian and thus the FX network is made up mainly of discrete nodes. Continuous nodes are used advisedly, as discussed in Chapter 6 in the section on managing OR with the model.

Every node in the network contains a Conditional Probability Table (CPT) that determines the strength of their relationship. The discrete nodes contain probability distribution over the states of the variables. These probabilities have to be encoded during the quantification of the network. There is a description comprising the name, the states and what every node clearly represents in the Node 
Description Table (NDT)as given in Tables 5.2 and 5.3.

The idea behind the NDT is to avoid any ambiguity in the meaning of the node. This is to prevent experts from assigning their own possibly different meanings and interpretations during the elicitation process. For example, the node frontoffice-system has three states: proper functioning (available and working correctly), malfunctioning (system errors, slow down, or working incorrectly), and not available or down. The accompanying description reads- the node refers to the availability and correct working of the front office. The proper functioning of this system implies the use of Straight Through Process (STP). Not available, or down, implies the use of manual methods to transmit the trade. The interface between the front-office-system and the back-office-system is considered part of the back-office-system. 


\begin{tabular}{|c|c|c|}
\hline \multicolumn{3}{|c|}{ INTERBANK SERVICES } \\
\hline \multicolumn{3}{|c|}{ Frequency Nodes } \\
\hline Node Name & Node Description & States \\
\hline Trade volume & Refers to the volume of trade transacted per week. & $\begin{array}{l}\text { Less than } 40,000 \\
40,000 \text { to } 100,000 \\
\text { More than } 100,000\end{array}$ \\
\hline Frontoffice_system & $\begin{array}{l}\text { Refers to the availability and correct working of the front } \\
\text { officesystem. Proper functioning implies use of STP and } \\
\text { not available or down implies manual transmission or } \\
\text { intervention. The interface between frontoffice_system } \\
\text { and backoffice_system is considered part of } \\
\text { frontoffice_system. }\end{array}$ & $\begin{array}{l}\text { Proper functioning (available }+ \text { working } \\
\text { correctly) } \\
\text { Mal functioning (system errors, slow } \\
\text { down or working incorrectly) } \\
\text { Not available/down }\end{array}$ \\
\hline Backoffice_system_slow_downtime & $\begin{array}{l}\text { Backoffice_system downtime comprises both slow and } \\
\text { downtime before some major currency cut off times. The } \\
\text { slow down time are the uncontrolled ones. Controlled } \\
\text { ones are not considered here. }\end{array}$ & $\begin{array}{l}\text { Less than } 5 \text { minutes (includes non } \\
\text { downtime) } \\
5 \text { to } 30 \text { minutes ( slow time, downtime, } \\
\text { unavailable or working incorrectly) } \\
\text { More than } 30 \text { minutes. }\end{array}$ \\
\hline Backoffice_system_fail time & $\begin{array}{l}\text { Refers to the time of backoffice_system failure. Failure } \\
\text { can occur at a critical time (some hours or minutes) } \\
\text { before cut off times of some major currency or non } \\
\text { critical time. }\end{array}$ & $\begin{array}{l}\text { Not Critical } \\
\text { Critical }\end{array}$ \\
\hline Trade Capture & $\begin{array}{l}\text { Refers to whether trade input was done in a correct and } \\
\text { timely manner or incorrectly or untimely. Errors include } \\
\text { wrong counterparty, wrong side of trade, value date, and } \\
\text { rate. }\end{array}$ & $\begin{array}{l}\text { Correct }+ \text { timely } \\
\text { Incorrect or untimely }\end{array}$ \\
\hline SSI_in place & $\begin{array}{l}\text { Whether there is a Standing Settlement Instruction in } \\
\text { place or not. }\end{array}$ & $\begin{array}{l}\text { Yes } \\
\text { No }\end{array}$ \\
\hline Trade type & Whether trade is of type standard or non standard & $\begin{array}{l}\text { Standard (Spot, Forward, Swaps) } \\
\text { Non standard (Options, NDFs etc) }\end{array}$ \\
\hline Gross or Netting & $\begin{array}{l}\text { Refers to whether trade transactions are to settle Gross or } \\
\text { should be Netted }\end{array}$ & $\begin{array}{l}\text { Gross } \\
\text { Netting }\end{array}$ \\
\hline Confirm matching & $\begin{array}{l}\text { Deals with the activity of confirmation matching before } \\
\text { trades are settled. Note, if SSIs are in place, confirmation } \\
\text { matching is done automatically and if not in place they } \\
\text { are done manually, which may introduces some errors. }\end{array}$ & $\begin{array}{l}\text { Not done (not sent, sent with no feedback } \\
\text { etc) } \\
\text { Done incorrectly (especially those done } \\
\text { manually e.g. phone etc) } \\
\text { Done correctly (matched) }\end{array}$ \\
\hline Quality of SI & $\begin{array}{l}\text { Refers to the ratio of Settlement Instructions which are } \\
\text { correct and current to the total number of settlement } \\
\text { instructions for a typical week. }\end{array}$ & $\begin{array}{l}\text { Excellent }(100 \%) \\
\text { Average }(98-100 \%) \\
\text { Bad (less than } 98 \%)\end{array}$ \\
\hline Paymentsystem_input_method & $\begin{array}{l}\text { Refers to the method (manual or automatic) of trade } \\
\text { payment instruction input into the paymentsystem after } \\
\text { they have been processed. }\end{array}$ & $\begin{array}{l}\text { Straight Through (STP) } \\
\text { Manual }\end{array}$ \\
\hline Trans_paymentsystem & $\begin{array}{l}\text { Refers to whether transmission to paymentsystem was } \\
\text { done correctly or incorrectly }\end{array}$ & $\begin{array}{l}\text { Correct and timely } \\
\text { Incorrect or untimely }\end{array}$ \\
\hline Vulnerability to failure & $\begin{array}{l}\text { Refers to the level to which a trade is liable to fail. It } \\
\text { depends on the trade capture and confirmation matching. }\end{array}$ & $\begin{array}{l}\text { Low } \\
\text { Medium } \\
\text { High }\end{array}$ \\
\hline Fails settlement & $\begin{array}{l}\text { Number of settlement failures in a typical week. Failure } \\
\text { here means delays, wrong payments, misdirected } \\
\text { payments, non payments and duplicate payments. It } \\
\text { depends on the vulnerability to failure, quality of SI, and } \\
\text { the input method into the transmission system }\end{array}$ & $\begin{array}{l}0 \\
1 \\
2 \\
3 \\
4 \\
، \\
\text { “ } \\
16 \\
17\end{array}$ \\
\hline
\end{tabular}

Table 5.2: Node description table - frequency of failures 


\begin{tabular}{|c|c|c|}
\hline \multicolumn{3}{|c|}{ INTERBANK SERVICES } \\
\hline \multicolumn{3}{|c|}{ Loss Event Severity Nodes } \\
\hline Node Name & Node Description & States \\
\hline Trade value & $\begin{array}{l}\text { Refers to the value (amount in EUR) of a trade } \\
\text { transaction. }\end{array}$ & $\begin{array}{l}\text { Less than } 200,000 \\
200,000 \text { to } 500,000 \\
500,000 \text { to } 1 \text { mill } \\
1 \text { mill to } 5 \text { mill } \\
5 \text { mill to } 10 \text { mill } \\
10 \text { mill to } 50 \text { mill } \\
50 \text { mill to } 100 \text { mill } \\
100 \text { mill to } 500 \text { mill } \\
500 \text { mill to } 2000 \text { mill }\end{array}$ \\
\hline Days delay & $\begin{array}{l}\text { Refers to the days of delay in re-settling a trade } \\
\text { transaction after it has failed. }\end{array}$ & $\begin{array}{l}1 \text { day } \\
2 \text { days } \\
3 \text { days } \\
4 \text { days } \\
5 \text { or more days } \\
\end{array}$ \\
\hline Interest rate regime & $\begin{array}{l}\text { Refers to the environment in which the trade is to settle. } \\
\text { The amount of compensation that has to be paid depends } \\
\text { on the settlement environment. }\end{array}$ & USD, Euro zone, GBP, JPY, CHF, etc \\
\hline Loss severity & $\begin{array}{l}\text { Refers to the severity of losses attributed to and payment } \\
\text { processing failures. This amount is given in EUR. }\end{array}$ & $\begin{array}{l}\text { Less than } 1,000 \\
1,000 \text { to } 5,000 \\
5,000 \text { to } 10,000 \\
10,000 \text { to } 50,000 \\
50,000 \text { to } 100,000 \\
100,000 \text { to } 250,000 \\
250,000 \text { to } 500,000 \\
500,000 \text { to } 750,000 \\
750,000 \text { to } 1 \text { mill } \\
1 \text { mill to } 5 \text { mill } \\
\text { More than } 5 \text { mill }\end{array}$ \\
\hline
\end{tabular}

Table 5.3: Node Description Table - severity of losses

\subsubsection{Model Structure Validation}

After the structure of the model has been determined, the model needs to be validated structurally. Two approaches are available for use 1) structural validation with experts and 2) structural validation due to Lauritzen et al.'s (1990) set theory or due to Pearl's (1988) d-separation principles (see Chapter 4).

Validation with the experts was carried out in an iterative process. Nodes were added and deleted during the process until some agreement was reached between the experts and the modeler that the model was an accurate reflection of the settlement process. Structural validation according to either the set theory or dseparation principle was not carried out here. This is because there is a built-in independence assumption, since the network was modelled in terms of causalities (causal network). Since the parents of the network were selected as direct causes of a given variable the local conditional independence conditions are automatically 
satisfied (Pearl and Russel, 2001).

\subsection{Quantifying the Network}

In general a combination of both subjective and objective is used in quantifying BNs. The emphasis here however, is on subjective data, since the historical data available was either found to be insufficient, unavailable or not in a form that can be easily encoded into the networks for some of the nodes. Subjective data was used for the entire frequency network although the terminal node, fail settlement has some objective data available. This data was set aside and later compared to the posterior probabilities of the terminal node during the model validation process. On the other hand, the severity network was quantified with objective data, with the exception of the days delay node, which had no historical objective data available. The objective data used and the process of obtaining subjective data are described in the next sections.

\subsubsection{Objective Data}

The structure of the severity network shows that the terminal node, loss severity depends on trade value, interest rate regime and days delay. The nodes trade value and interest rate regime are encoded directly with data from the trading books. The raw data consists of about 40,000 individual trades and 23 significantly different trading currencies, representing a trading period of one week. Details of these data include the trade date, value date, buy currency, buy amount, and sell currency. For confidential reasons, the raw data set is not provided in the Appendix. Processing of these data for final encoding into the network is described in Section 6.4.2.

Data for encoding the terminal node, loss severity was not directly elicited from the experts, but was computed according to European Interbank Compensation Guidelines (European Banking Federation, 2001). The reason is that during the testing of the elicitation process, the test expert actually computed the various losses quantities in accordance with European Interbank Compensation Guidelines. It was thus deemed reasonable that the experts be spared this enormous task, when the same computations could be done by the modeler. Details of this computation is also given in Section 5.5.3 


\subsubsection{The Elicitation Process}

Probability elicitation, as already mentioned in Chapter 4, is the process of obtaining estimates from domain experts. Our entire bayesian network requires about 11,155 individual probabilities. Fortunately, about 10,500 probabilities belonging to the severity network, are from the trade data (objective data) obtained from the trading books. The remaining probabilities (about 650) had to be elicited from the experts. These probabilities comprise both marginal and conditional probabilities.

Eliciting conditional probabilities is more difficult, especially if the probability is conditioned on several states. By applying the principle of divorcing of parents due to Olesen et al. (1989), described in Chapter 4, the number of states that the probabilities are conditioned on was reduced, which thus made the process easier for the experts. It should however be pointed out that in as much as divorcing parents and introducing intermediate variables lessens the difficult in eliciting conditional probabilities, it also increases the number of probabilities to be elicited.

A combined application of parametric elicitation, sensitivity analysis and removal of weak dependencies (see Chapter 4 for details) was able to further reduce the number of probabilities to be elicited from 650 to about 190. The use of parametric elicitation alone, for example, reduced the initial number of estimates from 486 to 27 for the terminal node, fails settlement of the frequency network. Sensitivity analysis uncovered the nodes days delay, quality of SI and vulnerability to failure as the most influential nodes of the severity and frequency networks respectively. A greater emphasis was therefore placed on these nodes during the elicitation process.

The elicitation of expert judgement followed a process which includes designing the instruments and procedures, testing of the instrument, conducting the real elicitation and finally editing and validating the estimates elicited. The components of the elicitation process are described below. 


\subsubsection{Protocol, Instruments and Procedures}

This subsection describes the protocol followed and the instruments used in our elicitation.

\section{Protocol}

The protocol used, referred to as Composite Protocol is a combination of some elements of the Stanford or SRI, Morgan and Henrion, Wallsten/EPA, and Cooke and Goosens protocols presented in Chapter 4. The Composite Protocol was formulated in order to have a comprehensive protocol that details out the various steps involved from start to end of the entire elicitation process. The essential elements are as follows:

- Identification and selection of experts (those for dry run and those for the real run),

- Preparation of a document that outlines the objective of the process and motivating the expert with the aims of the elicitation process,

- Structuring the uncertain quantities in an unambiguous way,

- Description of cognitive heuristics, biases and other relevant issues. The expert reads the document before the elicitation process.

- Definition of elicitation format document describing the exact questions and format for the expert's elicitation

- Dry run exercise describing the try out of the elicitation format document to a few experts ( This dry run may not foresee all the problems. However, since all in-house experts may share the same view, it is likely to bring out some interesting issues, which can be addressed during the real run)

- Expert training sessions describing the ingredients of training experts in preparing probabilistic assessments (describing for example, the elicitation technique and the quantities to be elicited)

- Expert elicitation sessions

- Feedback communication with the experts

- Documentation of the results 


\section{Questionnaire}

The main component of the instrument used in the elicitation process is a paper based questionnaire that was developed specifically for this purpose. Certain decisions were made prior to designing the elicitation questionnaire. One such decision is the type of information to be collected and the form (response mode) in which the experts' answers should be given. The type of information to be collected could be answers only, or answers plus the problem solving process. Our questionnaire collects answers only together with ancillary expert data since this is enough to encode our Bayesian network. Issues regarding the respond mode is discussed later in this section.

Another decision is whether simple probabilities or parametric distributions should be elicited. A combination of both was used. Simple probabilities were elicited for nodes where the burden on the experts was deemed to be minimal. Parametric distributions on the other hand was elicited for nodes where the burden on the experts was judged to be heavy. This is against the background that parametric elicitations is able to reduced the number of probabilities to be elicited drastically (see Section 5.4.2). Parametric distributions elicitation is usually used when the nature of quantities to be assessed suggest they follow a particular class of probability distributions. As indicated earlier in this section, parametric distribution elicitation is used for the terminal node of the frequency network. Here we assumed the "number of failures" follow a Poisson distribution and thus the parameters of this distribution were elicited. The assumption of Poisson distribution conforms to the industrial practice of describing the number of failures with either a Poisson or a negative binomial distribtion.

Having made these decisions, the questionnaire is developed to follow a structure which is consistent with the composite protocol. The questionnaire comprises the following elements: introduction; interview information; questions and answers or response mode; and experts background or ancillary information. These are described below.

\section{Introduction and Interview Information}

The introduction covers the purpose and objective of the elicitation exercise, details and instructions of the task for the experts and an explanation of the concept of cognitive heuristics and biases, and motivational biases. A typical introduction used for our elicitation is given in Appendix A. Interview information included the date of the interview, the place where the interview took place, the duration of the interview, and lastly, a description of the interview environment. Experts' ancillary information was also collected at the concluding part of the question- 
naire. This information deals with experts' background such as job position, the nature and type of work, previous work experience and how long the expert has been working at the present job.

\section{The Response Mode}

In the field of decision analysis, there are various methods for eliciting probabilities from experts. These methods include probability scales for marking assessments, frequency formats and gambles (see Morgan and Henrion, 1990; von Winterfeldt and Edwards, 1986). One method found particularly suitable in this context was that developed by van der Gaag et al. (2002) for eliciting probabilities in a cancer diagnosis study. This method was adopted, modified and used for this study. It combines ideas such as transcribing the probabilities to be assessed as fragments of text and providing a scale with both numerical and verbal anchors for marking the assessments. The fragments of text are stated in terms of likelihood rather than in terms of frequency, to prevent difficulties in assessments for conditioning context that is quite rare. The vertical response scale, with both numerical and verbal anchors is placed next to the fragment of text. Probabilities from the same conditional distribution are grouped together so as to encourage the experts to consider them simultaneously during the elicitation process.

One particular modification made to this method was to format the fragements of text (questions) so that contrasts among various variable states of a particular node becomes obvious. This helps the experts to clearly see the differences in change of state and thus to enable them to give appropriate answers. In addition, the format is such that similar themes are grouped at the top and the changing contrast are put at the bottom. The idea is to prevent wearing out the experts with too much reading load since reading the same scenario before answering every question on the same node would be repetitive, which might cause fatigue and irritation. Figure 5.4 gives an example of a typical questionnaire format. 


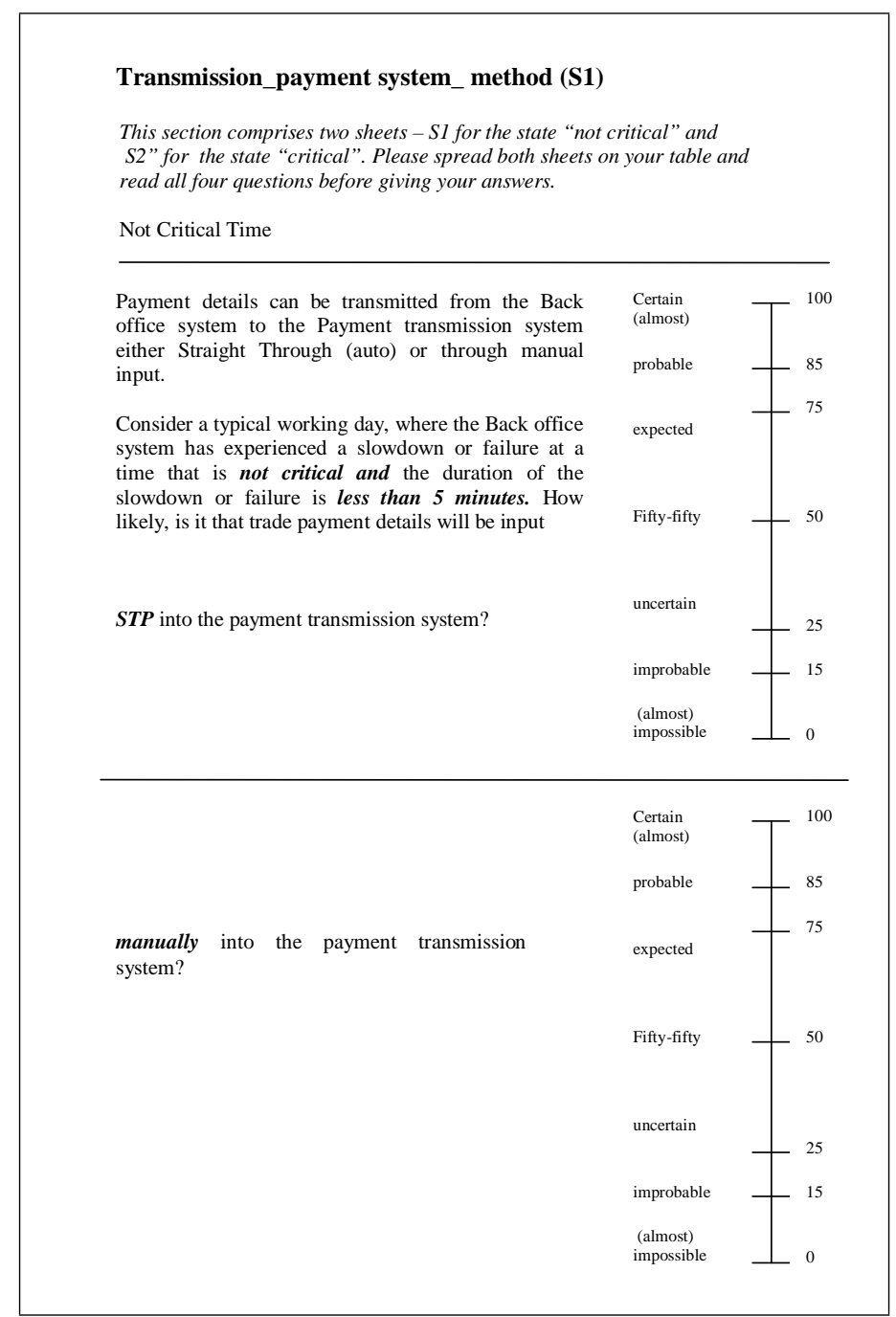

Figure 5.4: Typical questionnaire format

A further modification made was the use of a matrix format for nodes having several probabilities from the same conditional distribution. Samples of the matrix format are provided in Appendix A. The matrix table essentially contains questions covering all probabilities from the same conditional distribution and the various scenarios for a particular node. The advantage of the matrix format is that it gives the experts a one shot look at all the probabilities to be elicited from the same conditional distribution and the different scenarios. This affords them the opportunity to easily make comparisons among the various scenarios and to review their estimates without the difficulty of having to turn pages back and forth. Indeed, the test expert found this approach easier to use and recommended it for the real run. 
The matrix format was used for eliciting both point probabilities and probability distributions. Examples of the format is also given in Appendix A. For the elicitation of probability distributions, experts were asked to estimate a typical value and a range within which the typical value falls. An explanation of the terms typical value and range was provided on the matrix sheet. The typical value was interpreted as the frequently occurring number, and the range as the 5th and 95th percentile. Experts were also asked to give their estimates in absolute values instead of percentages. This was to cater for the difficulties and inherent errors in estimating small probabilities. For example, on average, 2 out of the 8000 daily number of trades fail. It would have been much more difficult for the experts to have quantified the corresponding probability of a trade failure, namely 0.00025 .

\subsubsection{Conducting the Elicitation}

Conducting the elicitation involved testing the elicitation procedure, doing the real run and countering bias during the real run. Experts had to be selected both for the testing and real run. The elicitation situation and mode of communcation also had to be defined.

Three experts with considerable experience and training in the domain were identified. One of the experts was used for the test run and the other two for the real run. These two experts provided estimates for FX and MM settlement networks separately. An expert is a person who has background in the subject area and is recognized by his or her peers, or those conducting the study, as qualified to answer questions. Questions are usually posed to the experts because they cannot be answered by other means. The experts' judgement is more than a guess. It is an informed opinion, based on the expert's training and experience (Meyer and Booker, 2001).

The elicitation situation adopted was a mixture of individual and interactive group. Individual because the two experts interviewed at the same time worked on separate networks and were also allowed to interact with each other. Personal interview (face-to-face) was chosen as the mode of communication in preference to mail or telephone. This mode was considered best for clarifying some ambiguity or misunderstanding with respect to some of the questions asked. Indeed, some modification was made to the structure of the network during the personal interviews because the experts found some variables difficult to quantify. This alludes to some of the advantages of doing personal interviews where such issues can be clarified and settled immediately. Mail or distant interviews do not afford that. 


\section{Testing}

The purpose of the test run is twofold: to obtain feedback information that can be used to revise the real elicitation and to give training to the project personnel (elicitor). Testing was carried out on one expert as mentioned earlier. Three components of the elicitation process were tested. These are introduction given to the experts, the elicitation procedures, and lastly the entry of data into the model for analysis or end use.

The first component familiarized the experts with the general steps and schedule to be followed, the questions and problems to be addressed, the response scale to use and the biases to which they may be prone. The second was to establish the duration for the elicitation, the appropriateness of the communication mode, the ease of understanding of the response mode, the clarity of the questions and the overall structure of the elicitation procedure among others. The last component was used to rehearse the entry of the collected data into the model. It also specifically revealed whether there was any discrepancy between the form of the elicited data and that necessary for the model.

A few observations made during the test run are: the need to give some form of training to the experts with regards to estimating probabilities and the use of the matrix format response mode; the need to provide clear and unambiguous meanings to some probabilities associated with certain nodes, which the test expert found difficult to estimate. This led to some nodes being modified or deleted outright. It was also observed that the test expert guessed probability estimates for sections where the expert's knowledge was limited.

The test expert had some initial difficulty with the matrix format, especially in formulating the questions in each cell. The test expert however, noted that with some initial training this format would be convenient, accurate and easy to use, since it emphasises the contrast in each scenario easily. Interestingly, it was observed that the expert was anchoring his estimates on recent events. This is not however a problem with the design of the elicitation process per se but an issue of anchoring bias. These observations were addressed during the real run.

\section{Real Run}

The real run consisted of six interviews scheduled over a period of eight weeks. Each interview lasted approximately 2 hours and focused on a small coherent part of the Bayesian network. The first interview was considered as a training session, which gave an introduction to the entire elicitation process and allowed the experts to work on sample questions. Working on sample questions made 
use of the element of learning in the sense that an expert would understand a technique much better after having used it once or twice in the assessment of different probabilities.

The training session reviewed the network and its probability tables, the basic ideas underlying the elicitation method (assessment techniques), the basics of probability theory, and the concept of bias and its effect on the results. Although the experts had been involved in the construction of the network structure, it was necessary to go through the network and its probability tables with them again. The general format of the fragments of text of the questions, the intended use of the response scale (marking of answers and writing of estimates), as well as the matrix format of questions were explained and demonstrated to the experts. The experts were also instructed to simultaneously take into account the different probabilities of the various states belonging to the same node when providing their estimates. In addition, they were also made aware of the fact that the elicitation is not only concerned about events that have occurred before but also about potential events that can occur under the described scenarios. Finally, they were informed that their estimates would be subject to analysis that will reveal the sensitivity of the network behaviour and if necessary the most influential probabilities will be refined later.

The subsequent interviews were quite straightforward. Each started with a short briefing by the elicitor and a reading of the introduction by the experts. The purpose was to help the experts refresh their memories about the elicitation process. Experts were then asked to mark the response scale and write a number close to their marks or to fill in the spaces provided where appropriate. The process was interactive with considerable dialogue between the experts and the project personnel, especially in sections where clarifications were needed. For those sections, where experts had limited information, they were encouraged to ask for help from other colleagues.

\section{Bias and Counters}

Bias, as mentioned in Chapter 4 is a deviation from reality or what we consider desirable. If not monitored and controlled in an elicitation process it can have an impact on the credibility of a project. Some bias tendencies can be anticipated before the process begins while others occur during the elicitation. Either way, both have to be monitored and countered. Bias was countered by following a protocol by Meyer and Booker (2001). The protocol has the following elements: 1) anticipate which biases are likely to occur in the planned elicitation processes,

2) redesign the planned elicitation to make it less prone to the anticipated bi- 
ases, 3) make the expert aware of the potential intrusion of particular biases and familiarize them with the elicitation process, 4) monitor the elicitation process for the occurrence of biases, 5) adjust in real time to counter the occurrence of biases, and 6) analyse the data for the occurrence of particular biases. However, not all these elements were needed.

Biases considered relevant to this work were Motivational and Cognitive (inconsistency, availability and anchoring). Motivational bias is driven by our human needs. This was anticipated and countered during the introduction stages by repeatedly assuring the experts that their answers would neither be subjected to scrutiny by supervisors, nor would they be used against them in anyway. Inconsistency may be due to fatigue leading to a faulty memory. To counter inconsistency, frequent breaks were scheduled during each interview session. In addition, definitions and assumptions used in the elicitation process, as well as the response scale, were reviewed at the start of each session. The presentation of questions and information in our questionnaire was structured hierarchically so that questions flow from general to the specific. This strategy, which stimulates and fires the experts memory associations (see Meyer and Booker, 2001) was used to counter availability bias.

Anchoring bias, the tendency to start with a first impression and then make only minor adjustments, was dealt with in three different ways: 1) experts were asked about extreme judgements before obtaining likely ones (see Cleaves, 1986), 2) experts were occassionally asked to describe how other experts might disagree with their responses, and 3) experts were asked to temporarily forget about recent events (see Boose and Shaw, 1989). Consistently asking experts to temporarily forget about recent events was found effective in dealing with the problem of anchoring responses on recent events. The experts found this approach very helpful.

\subsubsection{Validating the Estimates}

It is a known fact that validating an expert's opinion is a difficult thing to do. Any attempt should first seek to answer the question "what exactly do we intend to validate?" Is it to see 1) whether the elicited probability is the same as or close to the objective probability that is unknown, or 2) whether the elicited probability given by the expert is the same as the expert's knowledge?

There is a fundamental problem in attempting to address the first question. This is due to the fact that there is no objective distribution or probability to be esti- 
mated. The implication is that a particular subjective probability or distribution cannot be judged to be true or untrue, except of course, in some extreme cases where elicited probabilities are just unreasonable. One attempt in the literature (see Lindley et al., 1979) to deal with this problem requires that there be an objective assessor who will consolidate the results derived from the subjective experts. This model is currently being used by several banks in their operational risk self assessments, where the line manager provides probability estimates and a superior to the line manager, or the internal audit (assessor), validates the self assessments. The problem, however, with this approach is the implied assumption that the assessor is more objective than the expert.

The writer agrees with the opinion of Kandane and Wofson (1998) that since elicitation purports to represent the opinions of the person being elicited, and only that, any attempts at validation should use only other statements of belief by that person as data. A compromise to the method of elicitor and objective assessor, which seems useful is to elicit a quantity from a number of experts and then to aggregate these quantities into a single estimator and corresponding distribution through Bootstrap sampling and simulation (see Meyer and Booker, 2001). This is based on the premise that more than one person is a repository of the knowledge within a particular domain that is being elicited. This model unfortunately, can not be implemented if there are only a few experts in the domain being investigated, as was in the case study described here. However, a comparison of the estimates obtained from the two experts in the case study showed a high degree of consistency.

In OR self assessments, it is widely believed that experts may for obvious reasons, underestimate the frequency and severity of losses. This belief then transforms the problem of how close the elicited probability to the objective probability to a question of managing motivational bias - how can line managers be motivated to give a true and objective assessment of their risk? Managing motivational bias is therefore seen as a way to improve the quality of probabilities elicited, especially in operational risk. Managing motivational bias has already been addressed in earlier sections.

The question on whether probabilities elicited from the experts are the same as the expert's knowledge was dealt with by using the elements of validation (coherency, consistency and reliability checks) mentioned in Chapter 4. The specific approach used was to confront the experts with their estimates. Confronting the experts periodically with their answers for each coherent section of the network helps check the consistency or coherency and reliability of their estimates. This allows the experts to compensate for their natural biases and ensures the reli- 
ability of the estimates by way of the experts giving their overall statement of satisfaction. The approach is similar to the user-friendly JAVA interface created by Lau and Leong (1999), that checks possible biases and any inconsistencies in elicited probabilities by entering into dialogue with the expert until consistency is achieved. Besides confronting the experts with their estimates, the overall mathematical and statistical consistency (for example, probabilities adding up to one) of the estimates were also checked by the elicitor.

\subsection{Data Processing}

The data elicited from the experts, as well as those collected from historical sources had to be put in a form that can be encoded into the Bayesian network. This section describes how the data was prepared. It first looks at the data used for the frequency network and later that used for the severity network.

\subsubsection{Frequency Data}

As indicated earlier, expert judgement was elicited for all nodes in the frequency network. The quantities elicited include both probability estimates and parametric distributions (see Appendix B) ${ }^{1}$. The probability estimates were directly encoded into the frequency network. This is possible because the questionnaire were structured on the basis of the network.

The parametric distribution elicited for the terminal node (frequency of failure) has to be transformed before encoding into the network. To carry out the transformation, some assumptions were first made - the number of failures is assumed to follow a Poisson distribution and also our typical value is interpreted as the mean of the assumed Poisson distribution. This assumption is consistent with industrial practice and studies in operational risk that show that number of failures usually follow either a Poisson or Negative binomial distribution. The Poisson distribution is chosen over the negative binomial because the negative binomial was found to be overly dispersed for the elicited data (typical value, and the 5th and 95 th percentile).

A Poisson distribution has only one parameter, $\lambda$ (mean) and it is not always possible to satisfy both conditions of a prescribed mean and the two elicited quantiles (5th and 95th). It is however, possible to satisfy the condition of a prescribed

\footnotetext{
${ }^{1}$ the elicited data presented in Appendix B has been transformed for confidentiality reasons
} 
mean and one quantile by dropping the exactness requirement and using a condition "as close as possible" with respect to a predefined metric by determining $\lambda_{0}$ such that

$A *\left(\lambda-\lambda_{0}\right)^{2}+B *\left(P\left(X_{\lambda 0}>Y\right)-95 \%\right)^{2}$ is minimized.

$A$ and $B$ are weights emphasising the importance of the mean and the quantile value. $Y$ is the value that corresponds to the $95 \%$ quantile.

An application of this condition with various weights to the elicited data showed that the result is not very different from simply fitting a Poisson with its $\lambda$ equal to the typical value without any conditions (placing all emphasis on $\lambda$ ). The insignificant difference may be due to the fact that our data is somehow "under dispersed". This is true upon critical examination. Placing of full emphasis on the typical value is also consistent with the fact that when faced with the task of estimating statistics intuitively from data, subjects have been shown to be fairly accurate at guessing values of central tendency but not the variance of the data (Spencer, 1963; Swanson, 1966).

Having fitted a Poisson distribution to the elicited data, the various probabilities as specified in network were computed and encoded into the network. Some Bayesian network modeling shell (for example Hugins Expert) allows the specification of parameters of known distributions. This significantly lessens the burden on the modeler. The prior probabilities used for encoding the network are given in Appendix $\mathrm{B}^{2}$.

\subsubsection{Loss Severity Data}

The historic data used for encoding the severity network consists of trade information for 23 significantly different trading currencies. The various trade values were first converted to one common currency, the Euro (EUR), using the exchange rate that prevailed at each individual trade date. Data preparation for each of the individual nodes (days delay, interest rate regime, trade value and loss severity) are given below.

\footnotetext{
${ }^{2}$ the prior probabilities presented in Appendix B has also been transformed for confidentiality reasons
} 


\section{Interest Rate Regime}

Each trade in the data base has two sides; a buy side and a sell side. The sell side currency is the currency that has to be delivered and settled. The interest rate prevailing at the country where the sell side currency has to settle is chosen as the interest rate regime. For example, a trade with buy side EUR and sell side US dollar (USD) is represented as USD and is assumed to settle in the United States of America with its corresponding interest rate. Currencies that have some similarities in their exchange rate to the EUR and interest rates were grouped together and their interest rate was represented by a weighted average. Examples are the Australian dollar (AUD) and New Zealand dollar (NZD) represented as $A U D / N Z D$ and the the Russian rubble (RUB), Czeck krone (CZK) and the Swedish krone represented as $R U B / C Z K / S K K$. The representative interest rates were obtained from the internet websites of the corresponding Central Banks posted on 21.08.04. Table 5.4 shows the probability distribution of the various currencies and their interest rates.

\begin{tabular}{|c|c|c|}
\hline Currency & $\begin{array}{l}\text { Percentage } \\
\text { share }\end{array}$ & $\begin{array}{l}\text { Interest rate (\%) as at } \\
21.08 .04\end{array}$ \\
\hline USD & 37.95 & 2.000 \\
\hline EUR & 28.22 & 3.000 \\
\hline JPY & 9.62 & 0.002 \\
\hline GBP & 7.61 & 4.750 \\
\hline $\mathrm{CHF}$ & 5.05 & 2.000 \\
\hline AUD/NZD & 2.62 & 5.375 \\
\hline CAD & 1.83 & 2.000 \\
\hline RUB/CZK/SKK & 1.04 & 3.267 \\
\hline SGD & 1.02 & 1.100 \\
\hline SEK & 0.84 & 2.750 \\
\hline NOK & 0.69 & 1.750 \\
\hline TRL & 0.67 & 25.000 \\
\hline PLN & 0.63 & 6.000 \\
\hline HKD & 0.49 & 3.000 \\
\hline HUF & 0.46 & 18.000 \\
\hline DKK & 0.33 & 2.200 \\
\hline ZAR & 0.29 & 7.500 \\
\hline MXN & 0.15 & 7.100 \\
\hline $\mathrm{BRL}$ & 0.07 & 16.000 \\
\hline
\end{tabular}

Table 5.4: Currency distribution and interest rates 


\section{Trade Value}

To encode the various trade values into the network, the probability distribution of each currency was first determined. Two approaches were possible; use of parametric or non-parametric methods. Both methhods have their advantages and disadvantages. The later approach was used since the various trade values have fat tails and it was found expedient to let the data speak for itself, especially at the tails. An exploratory data analysis is given in Figure 5.5. Kernel density methods were used to estimate the various densities.

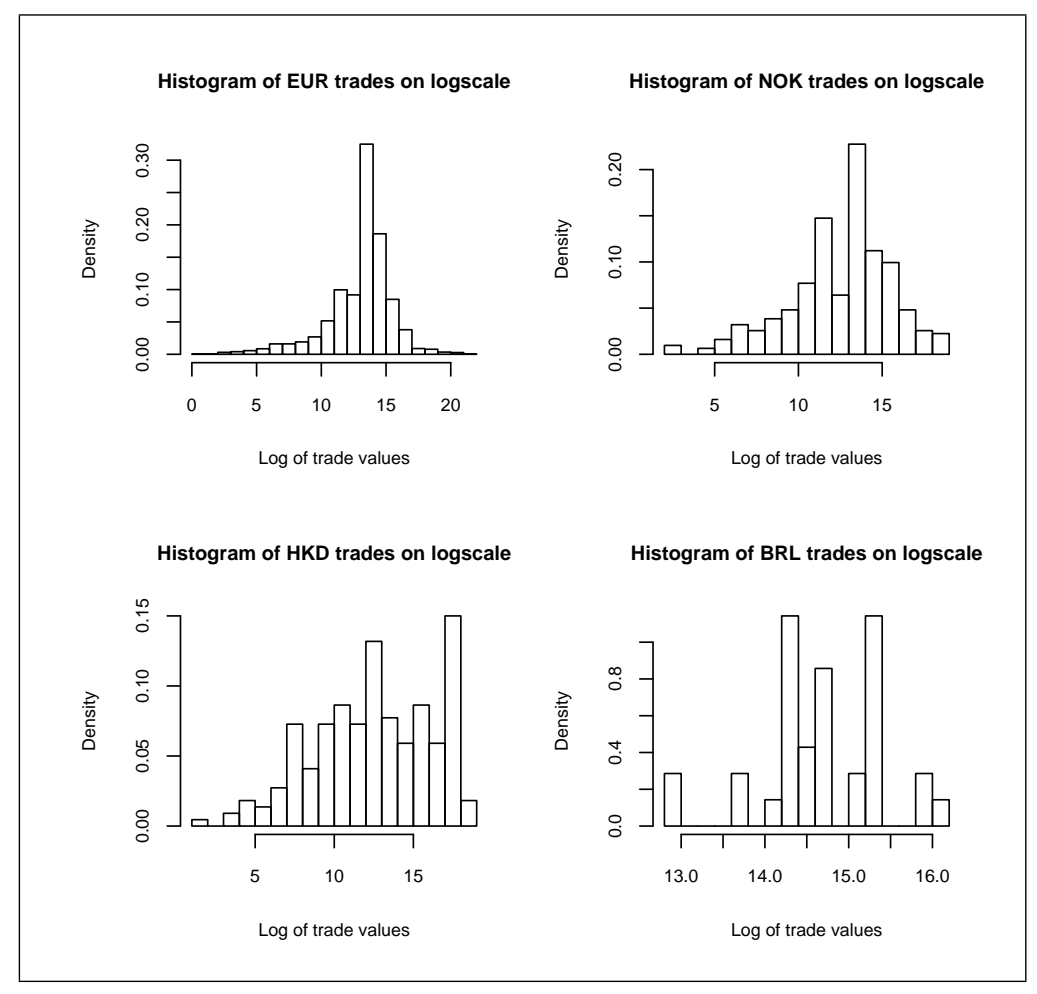

Figure 5.5: Exploratory graphs of some trade values

\section{Kernel Density Estimates}

In kernel density estimation there is always the issue of bias-variance trade off that has to be addressed. Two components that significantly affect this trade off are the bandwidth and the degree of local polynomial used. If the chosen bandwidth is too small, insufficient data will fall within the smoothing window and a large variance will result. If it is too large, some important features of the data may be distorted or lost. For any estimation problem, one may decide to either 1) apply a constant bandwidth to all observations, 2) choose a separate bandwidth 
for each fitting point or, 3) use a nearest neighbour bandwidth. Option two and three are classified as adaptive bandwidth selection.

A constant bandwidth for all fitting points is the simplest approach. Although simple, it may lead to problems of empty neighbour, which is most severe in tail regions. Choosing a separate bandwidth for each fitting point (see Brewer, 2000) is therefore preferable but computationally expensive, especially with numerous data points. The use of nearest neighbourhood bandwidth (see Loader, 1999) ensures that local neighbourhood always contains a specified number of points (solves the problem of empty neighbour) and also assigns different bandwidths to neighbourhood of points (adaptive bandwidth selection).

The degree of the local polynomial chosen also affects the bias-variance trade off. A higher polynomial degree will provide a better estimate, meaning less bias, but at the same time increases the variability of the estimates since the number of coefficient to estimate increases. This is in direct opposite to the effect of bandwidth. Experience shows that it is better to choose a low degree polynomial and rather concentrate on choosing a bandwidth. A local linear estimate usually produces a better fit, especially at the boundaries; a local quadratic reduces the bias but increases the variance, which can be a problem at the boundaries, and local cubic and higher orders hardly produce much benefit (Loader, 1999).

\section{Application}

The distributions of trade values data have fat tails. When the constant bandwidth approach was applied to the data set, the problem of empty neighbour was very pronounced for all the individual currencies with the exception of the Turkish Lire (TKL)and Brazilian Real (BRL). The use of separate bandwidths for each fitting point of our data was found to be computationally expensive (for example, Euro currency has over 17,000 observations). Use of locally adaptive fitting methods together with the nearest neighbour bandwidth method (see Loader, 1999, chp. 11) produced the most satisfactory results for the data set and was therefore used on all currencies except the TKL and BRL. For these two the constant bandwidth approach was used with an additional technique of cutting off the negative values of the estimate and rescaling the density to provide a positive support for the estimates. Figure 5.6 shows some examples of the kernel density estimates. 


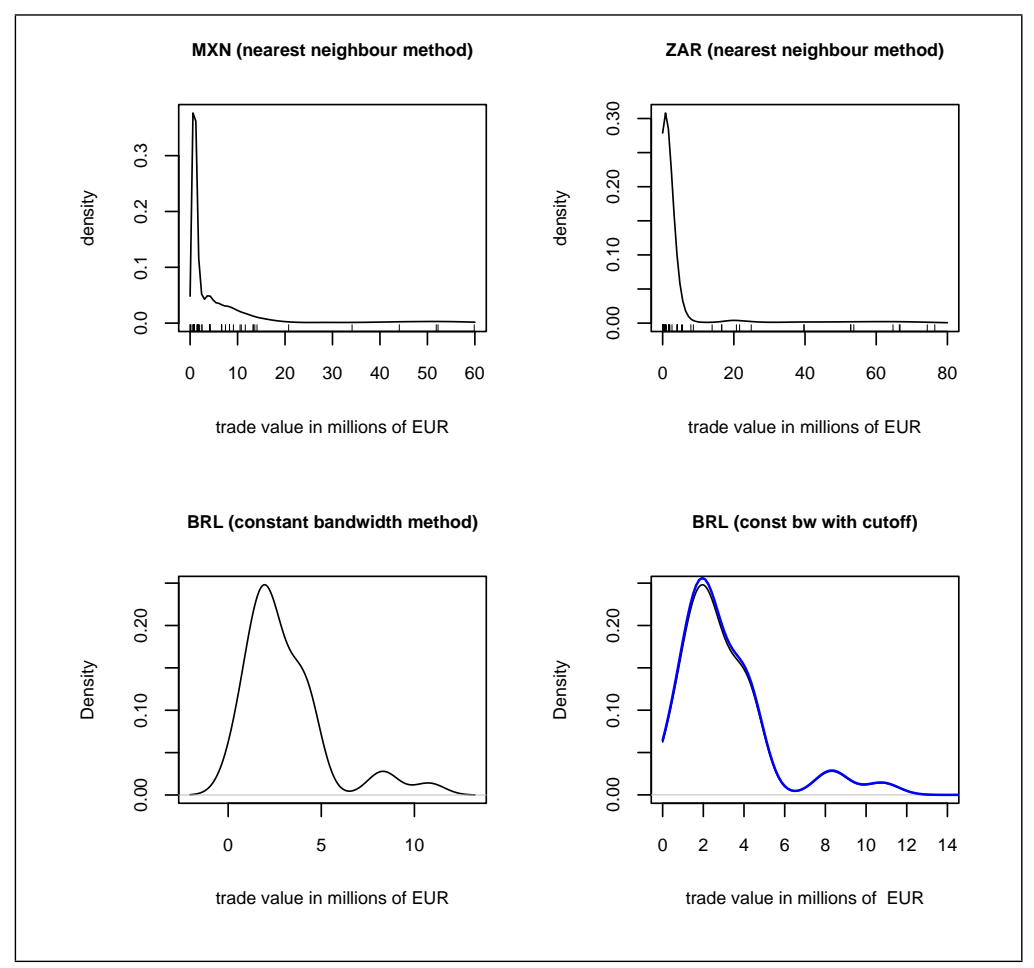

Figure 5.6: Kernel density estimation

Goodness of fit tests were carried out as follows: For a variety of smoothing parameters the density was estimated. A graphical comparison was then made between the empirical distribution function and the integrated density estimates. The emphasis of the comparison is at the tails, which is of much interest in operational risk. Quantile-quantile (qqplots) of the residuals obtained from the fit were also examined to assess the extent of lack of fit. For cases where there were competing models, and in which the graphs did not show clear distinctions, the Kulbach-Leibler (K-L) empirical discrepancy criteria was used. The essential part of the K-L empirical discrepancy criteria, which emphasises the fit in the tails of the density is given as

$\hat{\theta}=\operatorname{argmin}\left\{-\frac{1}{n} \sum_{i=1}^{n} \log g_{\theta}\left(x_{i}\right), \theta \in \Theta\right\}$

Some examples of goodness of fit tests are shown in Figure 5.7. 


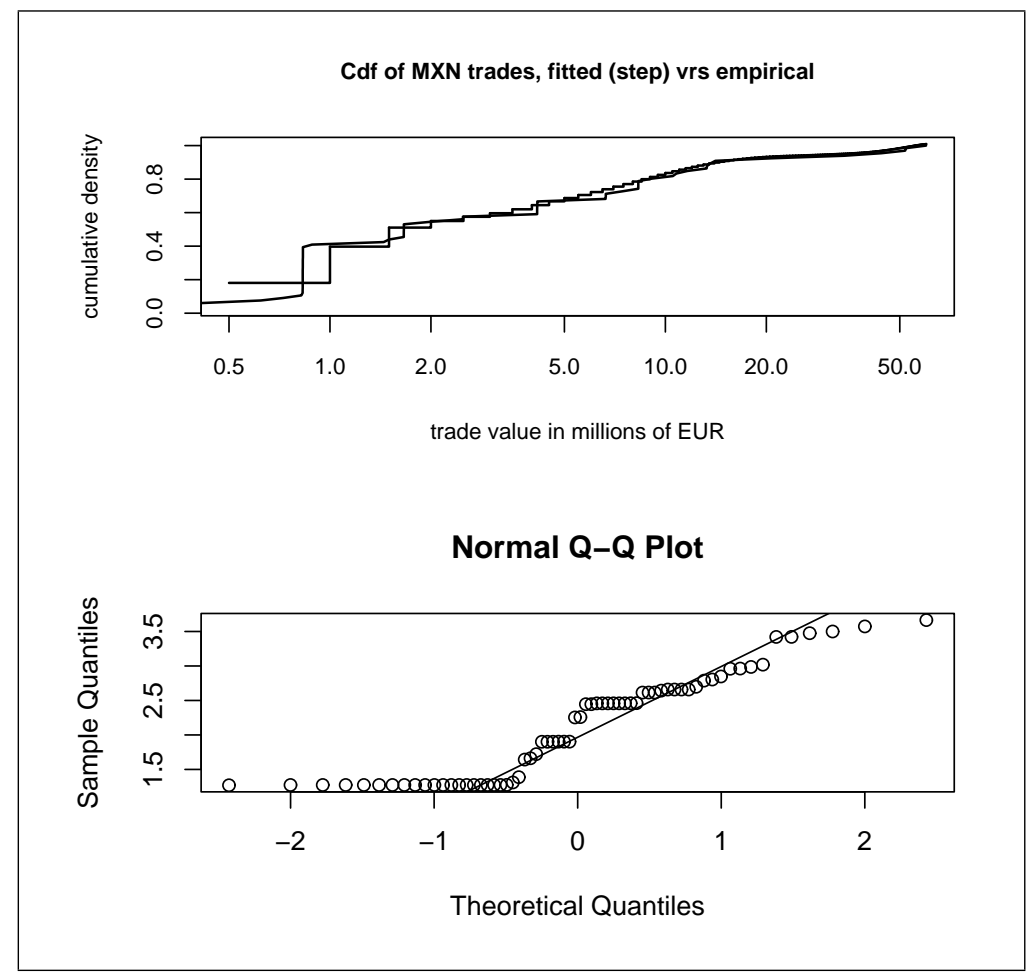

Figure 5.7: Assessing goodness of fit

\subsubsection{Loss Severity - Probability Computations}

Having obtained the estimated density of the various currencies, the probabilities needed for encoding the network are computed for the bin sizes pre-defined in the severity network. The computations are made in particular for two nodes: trade value and the loss severity nodes. Computations for the node trade value are quite straightforward (finding the proportional area under the curve covered by the defined bins) and will not be treated here. Computations of probabilities for the terminal node (loss severity) are discussed below.

\section{Loss Severity Node}

According to the severity network, loss severity depends on the trade value, the days delay, and the interest rate regime. The loss incurred if a trade fails to settle correctly, is computed as the product of the trade value, the days delay and the interest rate pertaining at the interest rate regime all divided by 360 days. This conforms with the European Interbank Compensation Guidelines (see European Banking Federation, 2001). Three points from the Guidelines are relevant for the 
computations: 1) compensations less or equal to 100 Euros are considered "de minimum" and not claimed, 2) an administration cost of 100 Euros is payable for failed trades and 3) in situations where the product of the trade value and the days delay is greater or equal to 200 million Euros, the compensation to be paid can be negotiated between the parties concerned.

The first provision is catered for in the network by cutting off losses less than 100 euros and normalizing the loss distributions. The last two provisions, for simplicity reasons, are not considered in the model. The interbank FX and MM market is a highly regulated market, unlike client FX market. As a result, the principal amount cannot be lost when banks trade with each other. The losses computed in the model is based on all currencies and corresponding interest rates, trade values, and an assessment of the days delay for a one-week trading period. The computed losses can therefore be considered as the universe of all possible losses that can occur for a one week trading period. The computations of the probabilities are given below.

Let $L$ represent the Loss severity, Int the interest rate in percentage, $V$ the trade or monetary value, $D$ the days delay, which takes values $d=1,2,3,4,5, \ldots$ and $k$ be a value representing $\frac{I n t}{360 * 100}$. Further, let, $\operatorname{lr} L$ be the lower bound of loss severity, urL the upper bound of loss severity, $\operatorname{lr} V$ the lower bound of trade value, and $u r V$ be the upper bound of trade value. The basic equation is

$$
L=V \cdot D \cdot k
$$

According to the severity Conditional Probability Tables $(C P T)$, we are looking for

$$
P(\operatorname{lr} L \leq L \leq u r L \mid \operatorname{lr} V \leq V \leq u r V)
$$

The conditional probability in Equation (5.2) can be expanded to

$$
\frac{P(\operatorname{lr} L \leq L \leq u r L \cap \operatorname{lr} V \leq V \leq u r V)}{P(\operatorname{lr} V \leq V \leq u r V)}
$$

The denominator $P(\operatorname{lr} V \leq V \leq u r V)$ of equation (5.3) can be computed as

$$
\int_{l r V}^{u r V} f_{v}(V) d v
$$


and the numerator $P(\operatorname{lr} L \leq L \leq u r L \mid \operatorname{lr} V \leq V \leq u r V)$ of equation (5.3) is expanded as

$$
\begin{aligned}
& \sum_{d=1}^{5} P(\operatorname{lr} L \leq V . d . k \leq u r L \cap \operatorname{lr} V \leq V \leq u r V \mid D=d) P(D=d) \\
= & \sum_{d=1}^{5} P\left(\frac{\operatorname{lr} L}{d . k} \leq V \leq \frac{u r L}{d . k} \cap \operatorname{lr} V \leq V \leq u r V\right) P(D=d) \\
= & \sum_{d=1}^{5} P\left\{\max \left[\left(\frac{\operatorname{lr} L}{d . k}\right), \operatorname{lr} V\right] \leq V \leq \min \left[\left(\frac{u r l}{d . k}\right), \operatorname{ur} V\right]\right\} P(D=d) .
\end{aligned}
$$

The first part of equation (5.7) can be written as

$$
\int_{\max \left(\frac{l r V}{d \cdot k}, l r V\right)}^{\min \left(\frac{u r L}{d . k}, u r V\right)} f_{v}(V) d v
$$

Putting it all together we obtain the final equation

$$
\frac{\int_{\max \left(\frac{\operatorname{lr} V}{d \cdot k}, \operatorname{lr} V\right)}^{\min \left(\frac{u r L}{u}\right)} f_{v}(V) d v}{\int_{l r V}^{u r V} f_{v}(V) d v} \cdot P(D=d) .
$$

The second part of the final equation $P(D=d)$ is catered for by the way the $C P T s$ in the Bayesian network is constructed. 


\subsection{Aggregating the Frequency and Severity Out- puts through Monte-Carlo Simulation}

The Bayesian networks developed produces two set of results (from the terminal nodes): 1) frequency of failures and 2) severity of losses. These two distributions are compounded through Monte-Carlo simulations to obtain a potential loss distribution, which shows the potential losses that can occur in the settlement process with its likelihood (see Figure 5.8). This section outlines the basic idea underlying the Monte-Carlo simulations. The results from the Bayesian networks and the Monte-Carlo simulations are described in Chapter 6.

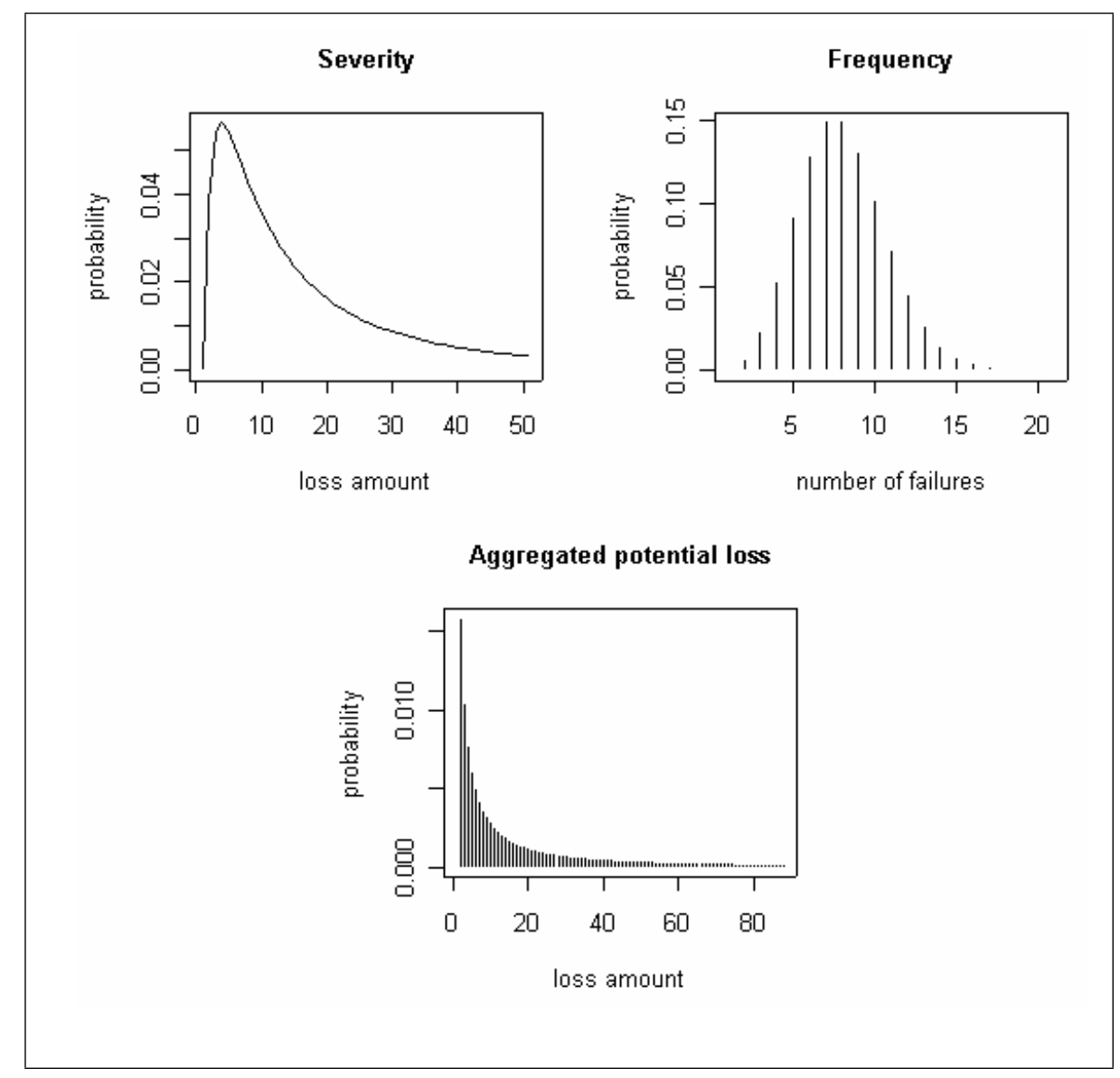

Figure 5.8: Aggregating frequency and severity distributions 
The idea underlining Monte-Carlo simulation is rather simple. To start the simulation, a decision is made on the number of iterations, say 100,000. The simulation proceeds as follows:

1. Generate a number of loss frequencies from the frequency distribution. Let's call this number $f d[1]$ for the first iteration.

2. Generate loss severities from the severity distribution for this iteration. The quantity of loss severity generated should be the same as the number of loss frequency generated above. That is, if the number of loss frequencies generated $f d[1]$ is 3 , we generate 3 loss severities from the severity distribution.

3. Add up the loss severities generated in 2) above for this particular iteration

4. Repeat the process for the total number of iterations, say 100,000.

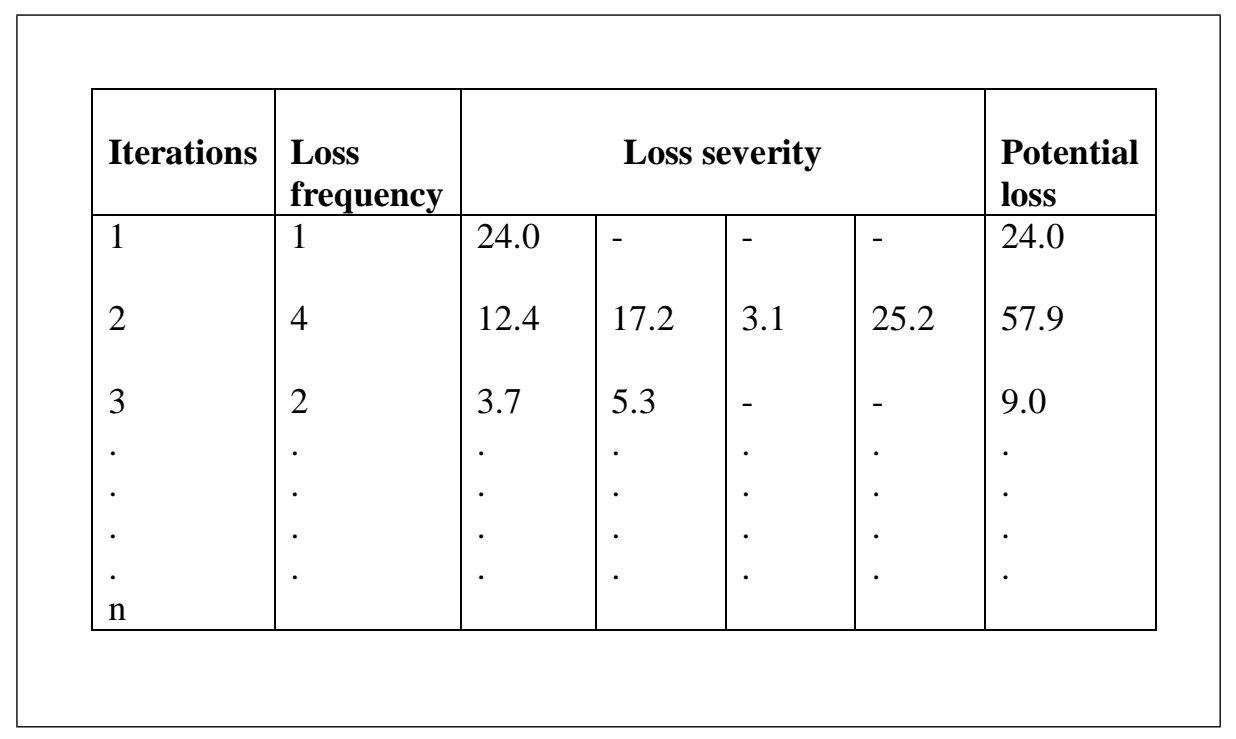

Table 5.5: Monte-Carlo simulations

Another way of executing this algorithm is by generating all frequencies and all the losses separately and later sorting and summing the generated losses into their various iterations. A random number generator is needed to generate the frequency and losses from the respective distributions. 


\subsection{Maintaining the network}

The Bayesian network constructed has to be maintained and periodically updated to reflect current conditions. The network structure and the initial probabilities should be updated periodically. Updating the network structure is particularly useful in operational risk management where the domain drifts over time (changing control environment and process flow). When the control environment changes, nodes can be added, deleted or modified to relect the current changes. New set of probabilities will also have to be elicited for the modified nodes.

Updating the initial probabilities used for encoding the network will require the establishment of a database based on the network structure. The database should have the same format as the CPTs so that historical data collected can be readily encoded into the Bayesian network periodically. In Bayesian networks, periodically updating the conditional probability distributions for a domain as observations are made is called sequential updating, adaptation or sequential learning.

One of the most common adaptation algorithms was developed by Spiegelhalter and Lauritzen (1990), who also introduced the notion of experience. Readers are referred to Cowell and Dawid (1992) and Olesen et al. (1992) for a mathematical treatment of adaptation. Experience is central to the process of adaptation. It is quantitative memory which can be based both on quantitative expert judgment and past cases. Experience can be formulated to either give equal weights to recent and older experiences or formulated to consider new observations more important than older ones and thus given more weight in the adaptation process. The later process is usually achieved by specifying a fading factor, which is the rate at which previous observations are forgotten. The algorithms for adaptation is implemented in most Bayesian network modeling shells. 


\section{Chapter 6}

\section{Results, Validation and Applications}

\subsection{Model Results}

The Bayesian network model developed and encoded in Chapter 5 was compiled to obtain some sample results. Compilation in the Hugin Expert modeling shell used was achieved through the junction tree algorithm described in Chapter 3. That is, the BN was first transformed into a junction tree and messages are then propagated through the tree to obtain the posterior probabilities. This chapter describes the results obtained from the frequency and severity networks. The aggregation of these results to obtain a potential loss distribution is also shown in this chapter. Included in this chapter is the assessment of the performance of the model. The last part of the chapter shows how the model can be used to manage operational risk in FX and MM settlement.

\subsubsection{Results - Frequency of Failure and Severity of Losses}

The frequency network with some posterior probabilities is shown in Figure 6.1. The probability distribution for the terminal node (hypothesis node) fails settlement shows that for a period of one week, the probability of having no trade failure is about $11 \%$, one failure is about $23 \%$, two failures is about $24 \%$ and three failures is about $18 \%$. These results are later compared to historical data.

Figure 6.2 shows the severity network with some posterior probabilities. The distribution of the node of interest, loss severity indicates that the most likely 


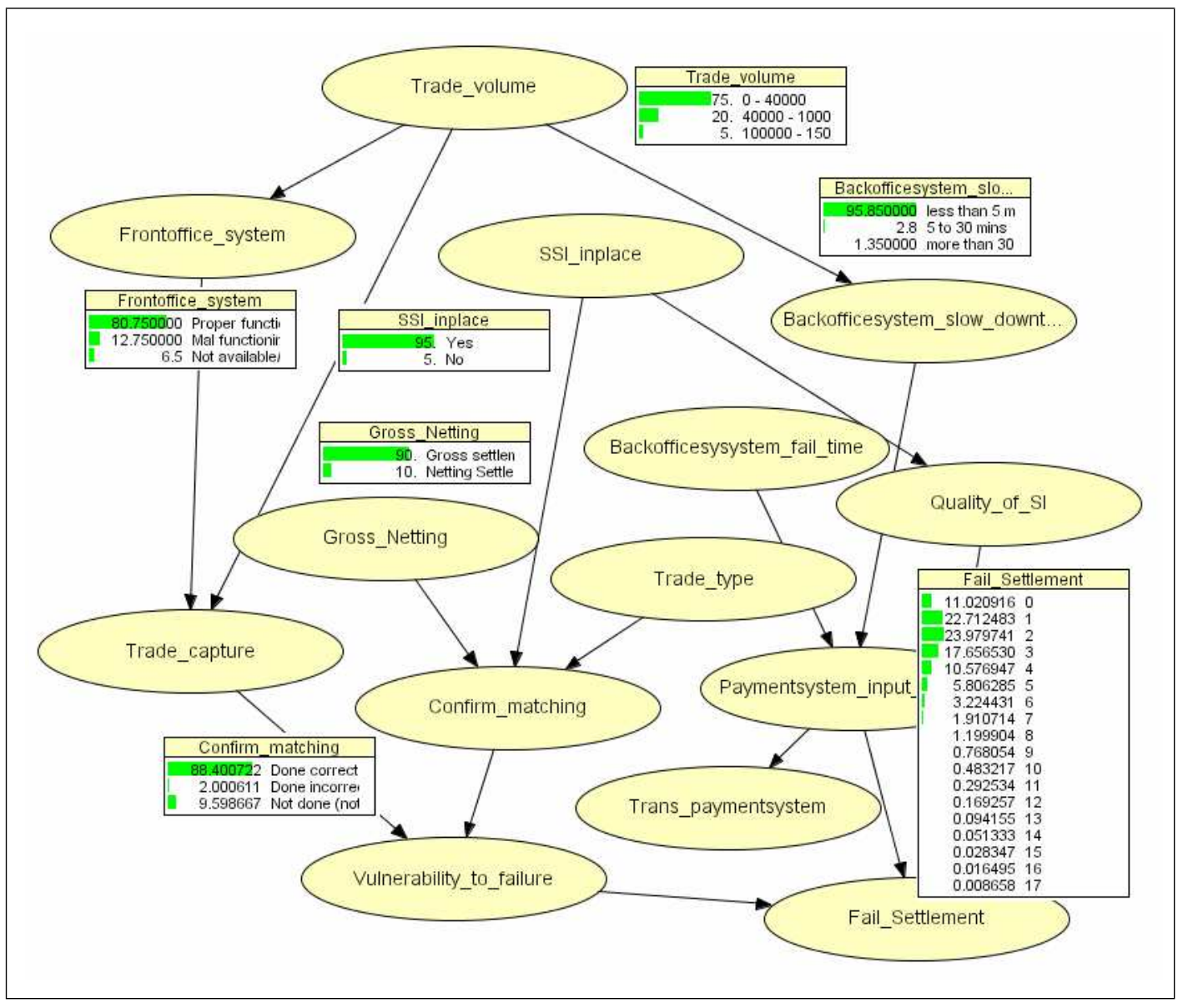

Figure 6.1: Frequency network showing some posterior probabilities

loss amount should given a settlement failure in a week is less than 1000 EUR. The corresponding probability of occurrence is $90 \%$. Other features are lossess of between 5,000 EUR to 10,000 EUR, 100,000 EUR to 250,000 EUR and 750,000 EUR to 1 million EUR occuring with probabilities of $1.46 \%, 0.129 \%$ and $0.005 \%$ respectively. 


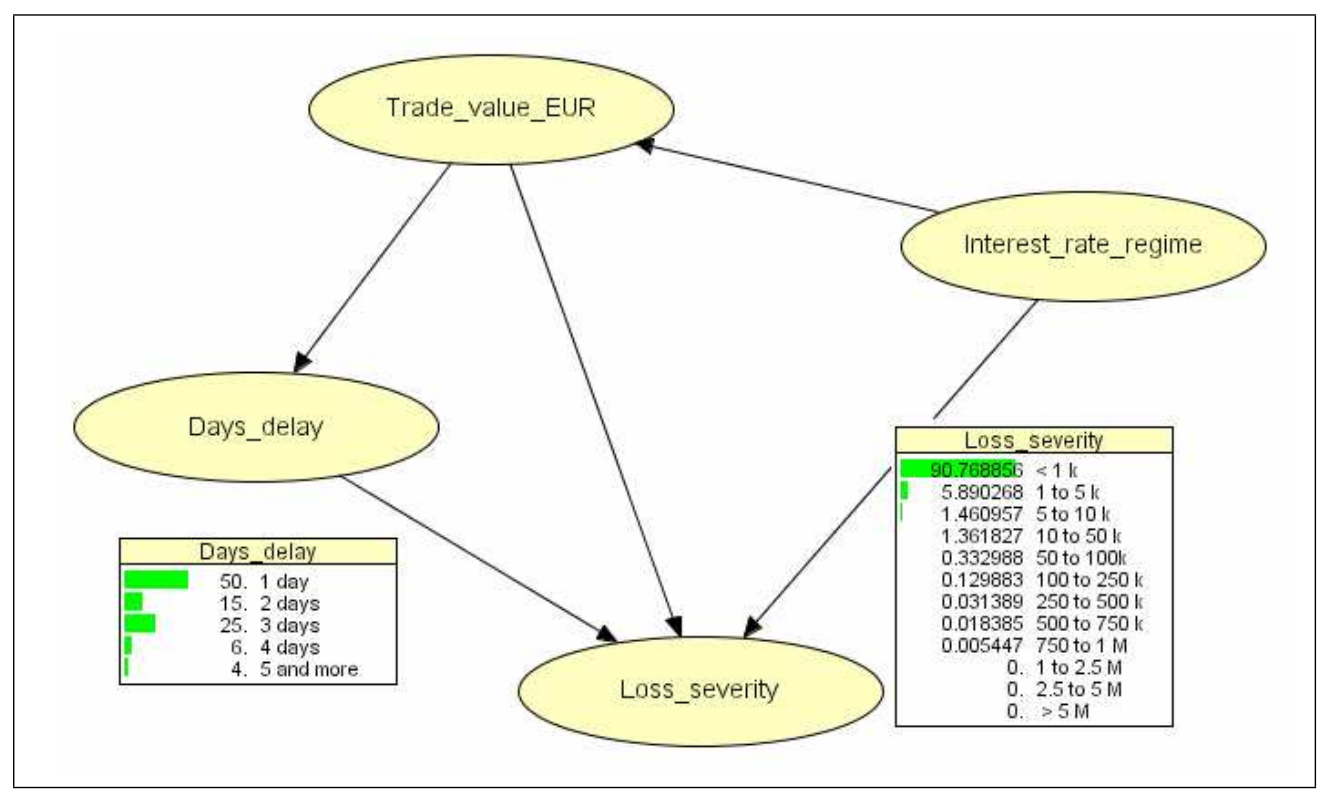

Figure 6.2: Severity network showing some posterior probabilities

\subsubsection{Results - Aggregated Potential Losses}

A summary of the results obtained from the aggregation through Monte-Carlo simulation is given in Table 6.1. A histogram of the potential loss is shown in Figure 6.3. The results indicate that the mean loss that can occur within a trading week is about 11,290 EUR with a variance of 136 EUR. The maximum potential loss that can be experienced is $3,142,000$ EUR. The potential loss distribution like the loss severity distribution also has a fat tail.

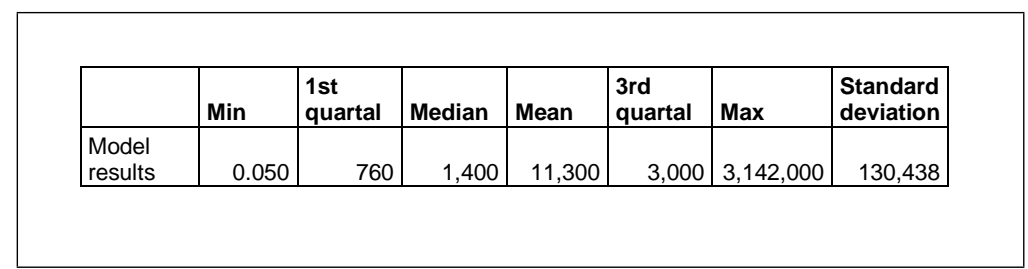

Table 6.1: Summary statistics of potential loss 


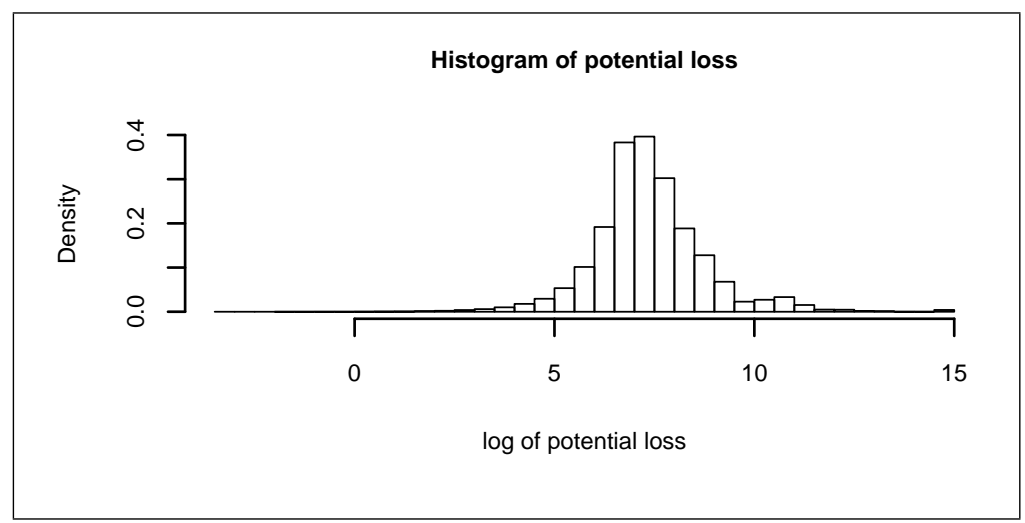

Figure 6.3: Histogram of potential losses

\subsubsection{Results - Operational Value at Risk}

Value at Risk (VaR) measures the worst expected loss under normal market conditions over a specific time at a given confidence level. It normally answers the question how much can be lost with $x \%$ probability over a preset horizon (J.P. Morgan and Reuters, 1996). It is normally denoted as $V a R$ at holding period $t$ and confidence level 100(1- $\alpha) \%$. For example, a weekly $V a R$ of 500,000 EUR at $5 \%(\alpha=5 \%)$ means only 5 chances in a 100 that a weekly loss bigger than 500,000 EUR occurs under normal market conditions.

Usually when the primary goal is to satisfy external regulatory requirements, the $(\alpha)$ or quantile is typically very small, for example $1 \%$. However, for an internal risk management model used by a firm to control its risk exposure, the typical number is around 5\%. In operational risk the regulatory value is fixed at $0.1 \%$, that is, the confidence level is at $99.9 \%$. Since the FX model is primarily for management purposes, the operational value at risk (OpVaR) is also evaluated at $95 \%$. A simple way of computing the OpVaR is to order the values obtained from the aggregated distribution (Monte-Carlo simulations) in descending order. The quantiles are then obtained by dividing the position of each value by the number of runs. Table 6.2 shows some OpVaR values. The significant difference between the OpVaR value at $99.5 \%$ and $99.9 \%$ also confirms the fat tail of the distribution.

The operational risk expected loss is the mean of the aggregated potential loss distribution and the unexpected loss at the $i^{\text {th }}$ percentile is the difference between the upper $i^{\text {th }}$ percentile and the mean of the potential loss distribution. The expected loss and the unexpected loss at both $99.9 \%$ and $95 \%$ are given in Table 
6.2. The unexpected loss of 2,823,000 EUR at 0.1\% (99.9 quantile) means there is only a 0.1 chance in a 100 that a weekly loss bigger than 2,823,295 EUR occurs under normal trading or settlement conditions.

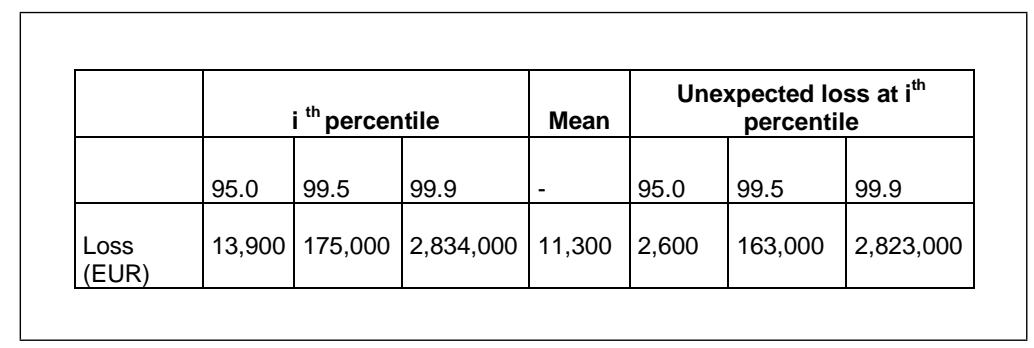

Table 6.2: Operational risk unexpected loss

\subsection{Model Validation}

The FX and MM settlement model developed is validated through a series of comparisons carried out in this sections. The model's output - distribution of frequency of failure and severity of losses are compared with historical data. The aggregated potential loss distributions simulated through Monte-Carlo methods for both the model and historical data are also compared. The historical data used for the comparison are first described.

\section{Historical Loss Data}

The historical loss data used for comparison is actually simulated from a banks real loss data for FX and MM settlement processes. The simulation was done in order to respect data privacy laws. It is however believed that the integrity of the data was somehow kept during the simulation process to enable a reasonable comparison to be carried out. The data covers a total period of about two years (2002 - 2004).

The historical data is truncated. As indicated earlier in Chapter five, the European Interbank Compensation Guidelines stipulates that settlement losses less or equal to 100 Euros are not claimed. This gives a truncation point of 100 EUR. There are however a few data points with loss values less than 100 EUR found in the data set. Additionally, the experts indicated during the elicitation process that at the beginning of the historical data collection exercise, losses less than 1000 EUR where not recorded. This amount was later reduced to 500 EUR at some point in time. Unfortunately, the time for this change is not documented. 
Thus the dataset could possibly be truncated at 100, 500 or 1000 EUR. To simplify the comparison, the model results are also truncated at 100, 500 and 1000 EUR for the loss severity data before comparing them with the historical loss data.

\subsubsection{Comparison of Results - Frequency of Failures}

The frequency of failures from the model results have a weekly time span. In view of this, the historical frequency of failure data is first regrouped to also have a weekly time span. For ease of comparison, a Poisson distribution was fitted to the historical data. The graphical comparisons are shown in Figure 6.4

From Figure 6.4 the chances of experiencing one or zero number of failures in a trading week are higher for the historical data (about 30 and 40 percent respectively) than for the model results (about $10 \%$ and $25 \%$ respectively). On the other hand, the chances of experiencing two or more failures are higher for the model results than the historical data. Additionally, whereas the probable number of failures for the historical data terminates at five, that of the model results terminate at seventeen, but with a small chance of occurrence $(0.028 \%)$.

These results are not surprising but confirm the fact that the model includes various scenarios, which might not have occured before but do have the potential of occurring. An example is the estimation of probabilities for rare events like the number of failures exceeding 10 failures per week when the historical loss data has never recorded more than 10 failures per week. Another example is the estimation of probabilities for the states "proper functioning", "mal function" and "not available" for the node "front office system" under the scenario of "trade volume" between 100,000 and 150,000. Such a weekly trade volume has never been recorded in history. These probabilities although small do influence the final results. The inclusion of such scenario analysis prevents estimating as zero the inherent operational risk within certain process with little or no available history of losses (see van den Brink, 2002, chp. 2). 


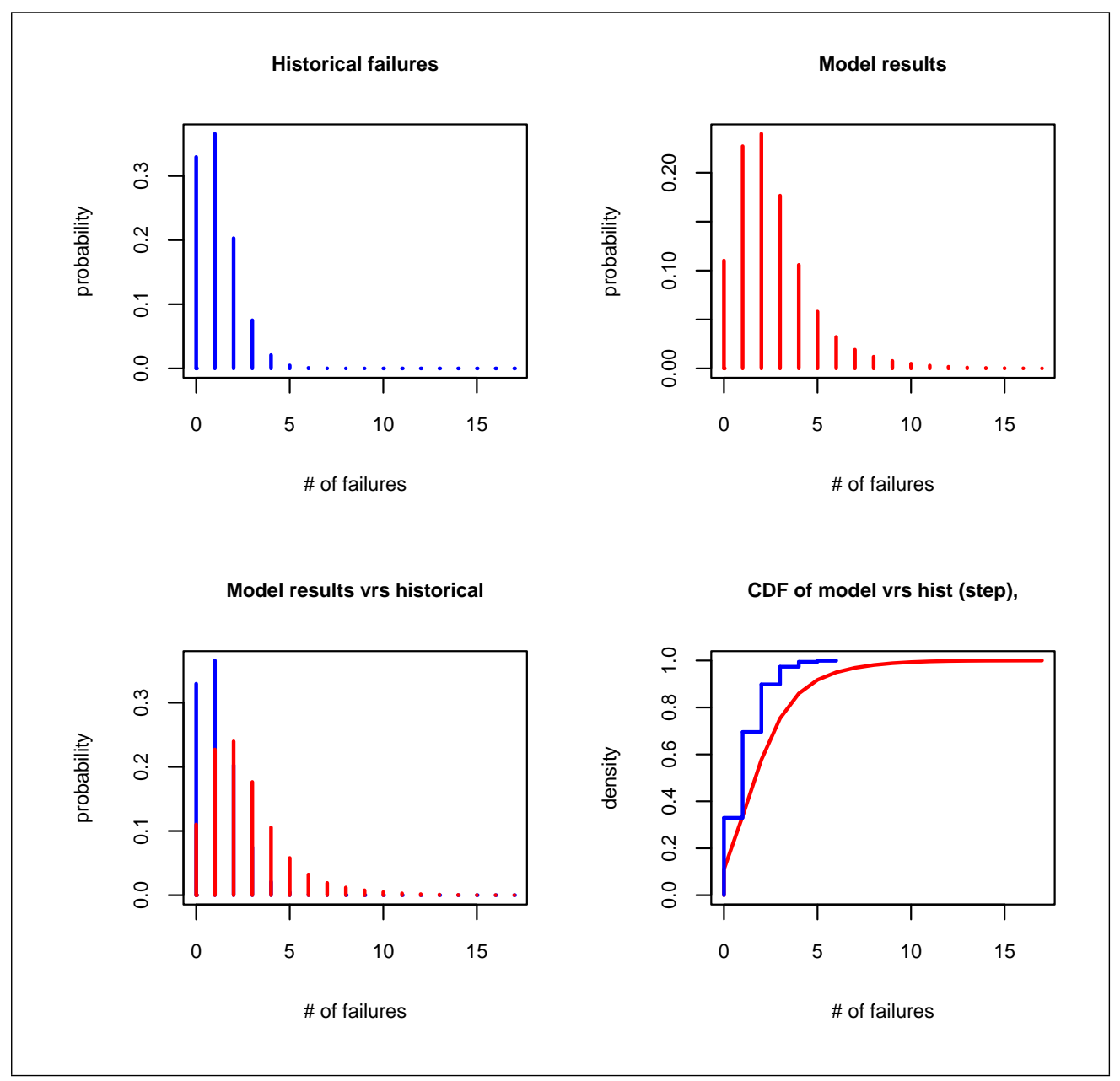

Figure 6.4: Comparison of frequency of failure

\subsubsection{Comparison of Results - Severity of Losses}

Comparisons are made for both data sets truncated at 100, 500 and 1000 EUR. To help with the comparison, a lognormal distribution was fitted to the historical loss data. Thereafter, a comparison of the commulative density function (cdf) of the model results and the historic data is made (see Figure 6.5). Furthermore, quantile-quantile plots (qq plots) of the two data sets (see Figure 6.6) is also made. The idea behind the qq plot is to first obtain corresponding values of the same quantiles for both cdfs and later plot them against each other. If both are comparable, they should follow a 45 degree inclined straight line.

The cdf plots show that losses from our model results are higher than those from 
the historical data. The model results also have a fatter tail. It is obvious from the qq plots that the two distributions are different for all three cases(truncation at 100, 500 and 1000 EUR). For all three cases the historical losses are higher than the model result for very low losses but the trend changes after some point. The only difference being the change point.

This underscores the fact that the model emphasises high losses whereas the historical data, which has few observations emphasise low losses. In addition, the losses computed and used in the BN comprised all possible scenario combinations of trade value, applicable interest rate and days delay. The historical losses can thus be seen as a subset of these scenario combinations. One example of a scenario included in the model but has never been recorded in history is the estimation of probability of a trade value of between 500 million EUR to 2 billion EUR failing to settle for more than 3 days. Indeed, such an amount has never been recorded to have failed for even a day.

In modeling Bayesian networks it is possible for results to be way off the expected. In such a situation one technically always has the option of going back and assessing which information needs to be rethought if the estimates look unrealistic in a $\mathrm{BN}$.

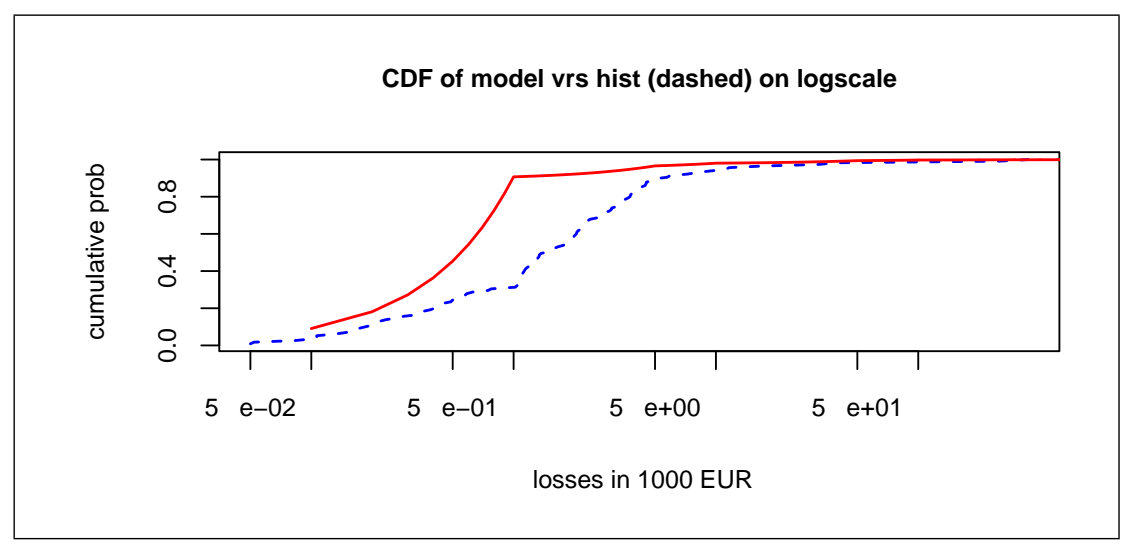

Figure 6.5: Commulative distribution function of losses (logscale) 


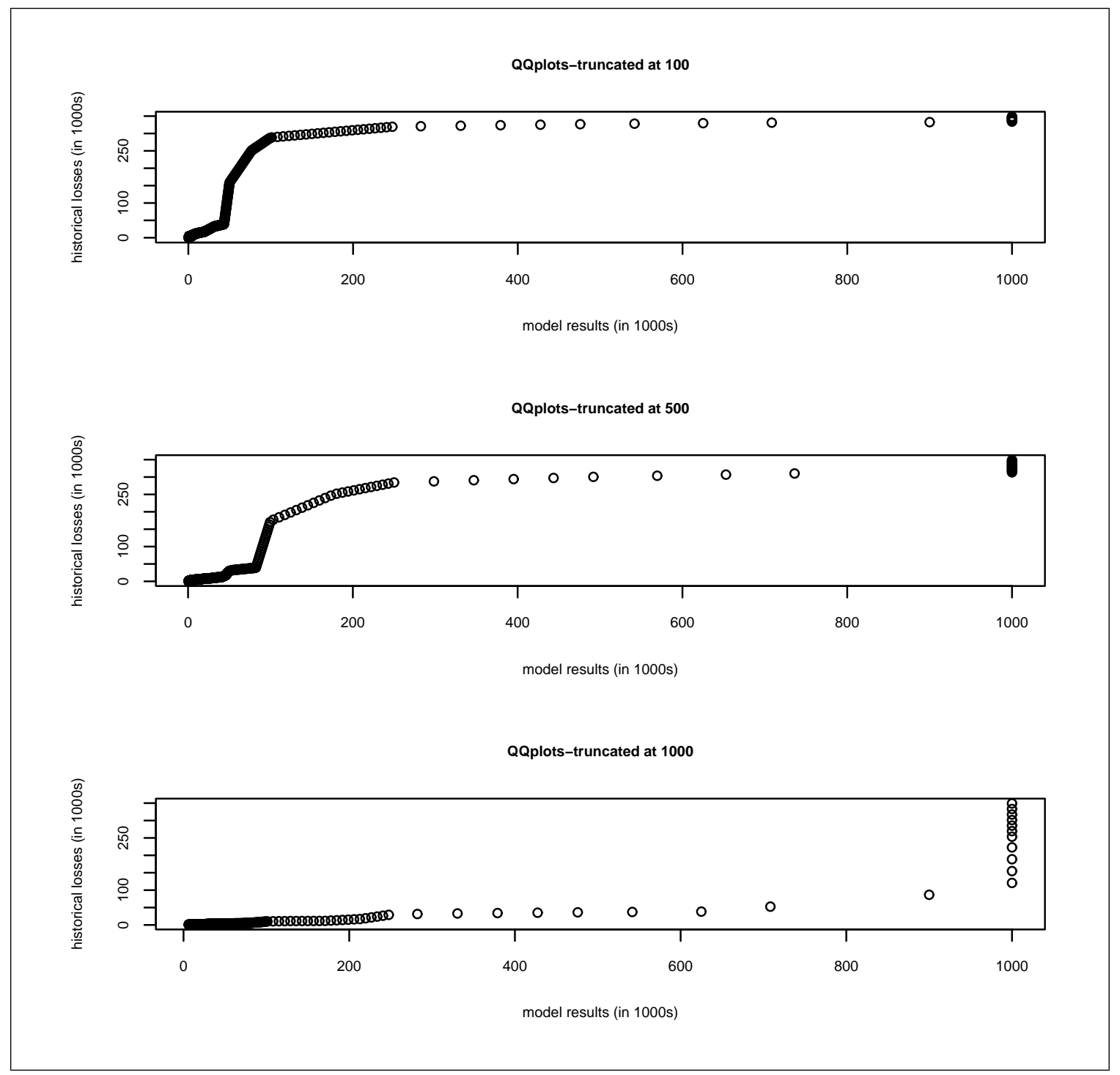

Figure 6.6: QQ plots of losses with truncations at 100, 500 and 1000 EUR

\subsubsection{Comparison of Results - Potential Losses}

Comparisons are made again for the three truncation points. First the summary statistics of the two distributions (model results and historical data) are provided in Table 6.3. These results are obtained through the Monte-Carlo simulations mentioned in section 6.4 (for the historical data, the simulation is generated from the Poisson and lognormal distribution fitted to the historical frequency of failure and historical losses respectively). We compare the cdfs plots on logscale followed 
by a qq plot of the two distributions. The qq plots are broken up into two quantile ranges so that we do not miss some of the fine features of the plots.

The summary statistics show that for all three truncation cases, the model results have a higher maximum potential loss, $99.5 \%$ and $99.9 \%$ quantile values than the historic potential losses. In addition, the first three quartals (1st, median, 3rd quartal) of the model results, except the 100 EUR truncated data set are higher than the historical results. In general, the cdf plots in Figure 6.7 show that the model results have fatter tails than the historical results. A careful look at the cdfs of the three truncation cases indicate indicates that the model results and historical data for truncation at 1000 EUR compares most favourably than the others. This is not surprising especially when our model emphasises high losses.

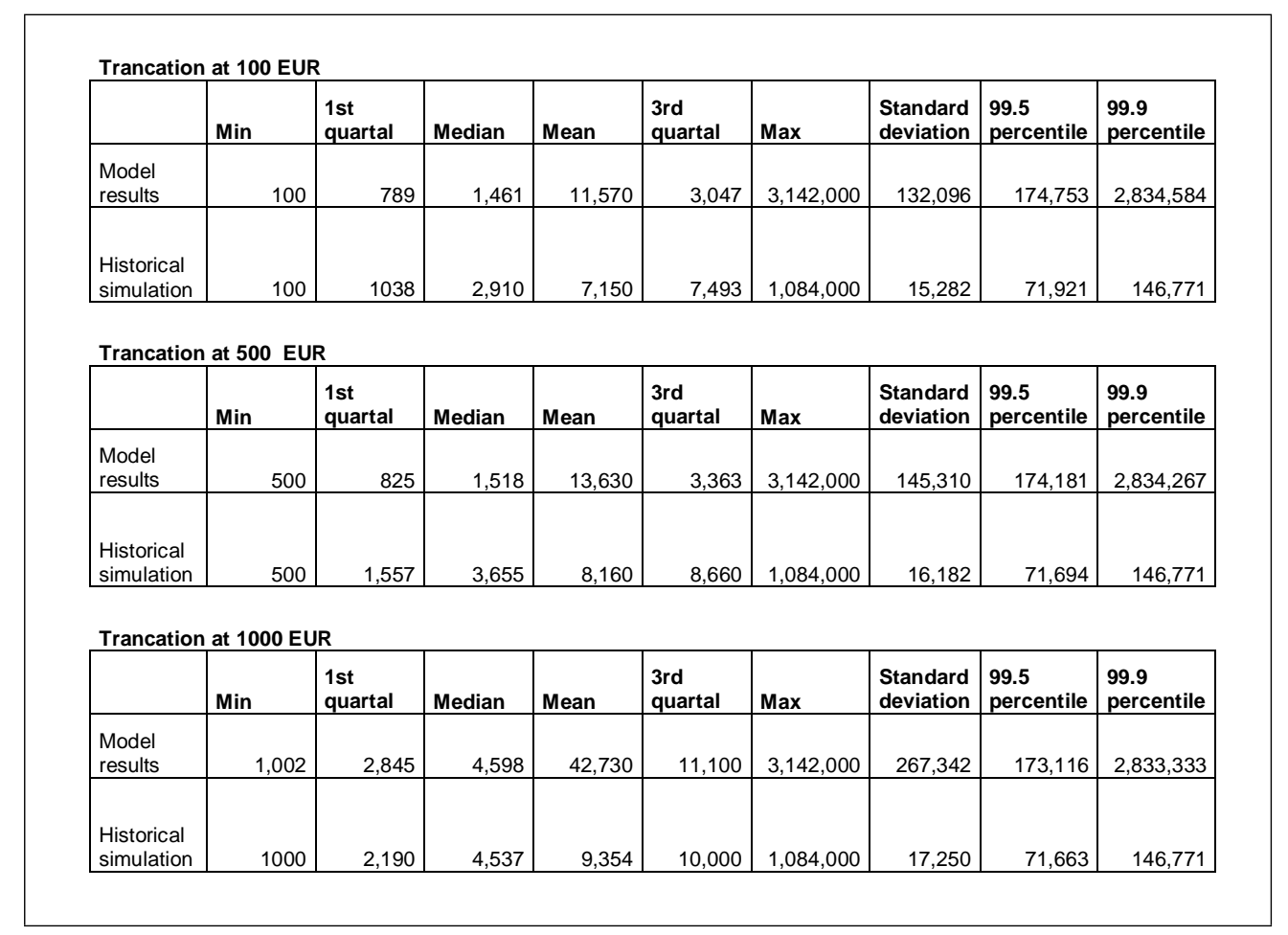

Table 6.3: Operational risk unexpected loss 


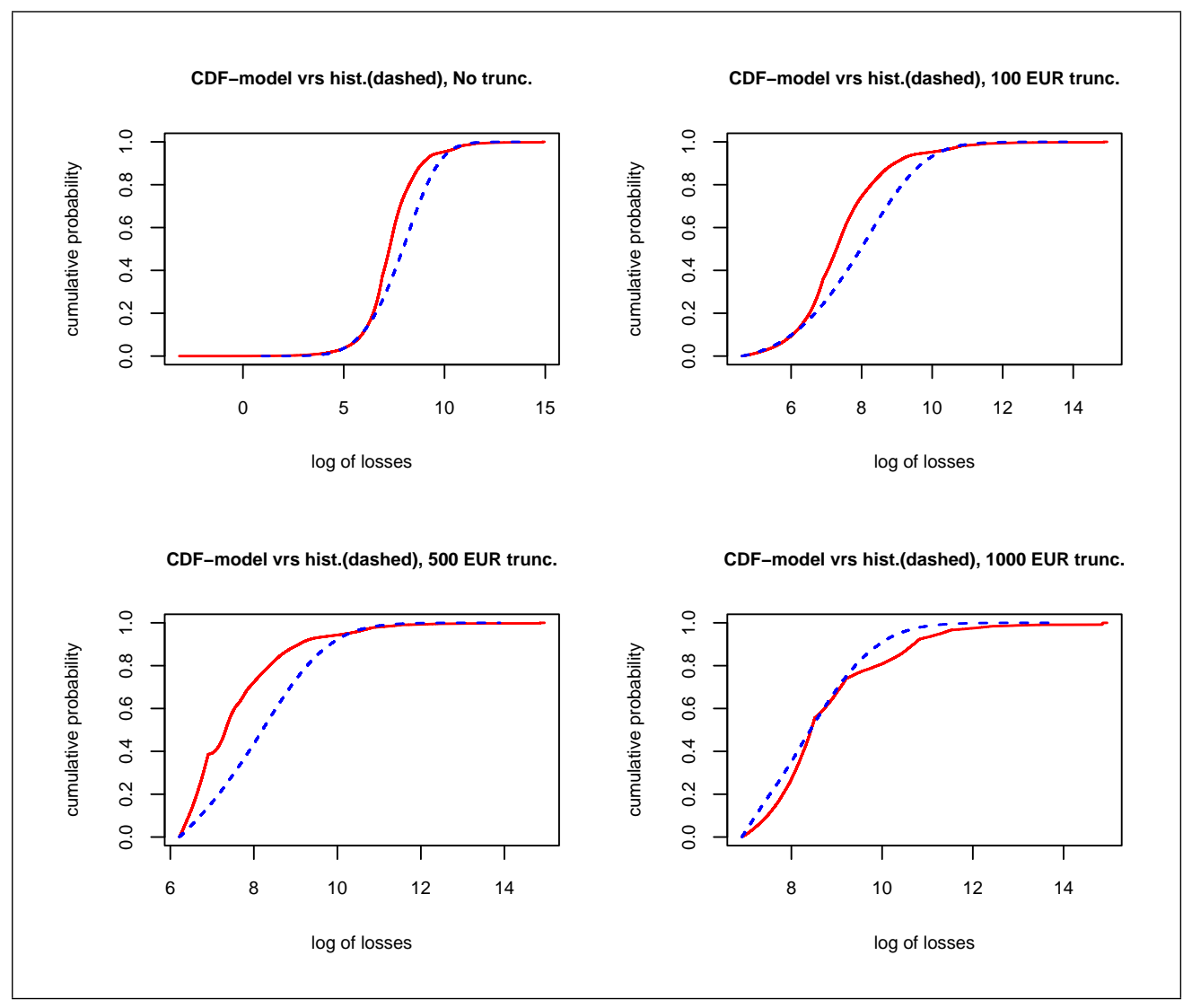

Figure 6.7: Potential loss - cdf comparison

The qq plots in Figures 6.8 and 6.9 show that overall (quantile 0 - 1), the model compares badly with the historical results for all cases of truncation. A detailed look at quantile plots (quantile 0 - 0.97) however, indicates a better comparison - meaning the main body of the two distributions up to about the 0.97 quantile are comparable. The significant difference is really at the tails of the distribution, where the model results are significantly higher than the historical distribution.

A critical examination of the quantile $0-0.97$ plots indicate that potential loss from historical simulation are higher than the model results only up to a particular loss amount. After this amount there is a change in the trend and the models results become higher than the historical losses. That is, as one moves further away from the body of the distribution, the model results become higher than the historical results. The change points are different for each of the cases, for example, about 10,000 EUR for truncation at 1000 EUR, 20,000 EUR for truncation 500 EUR and 25,000 EUR truncation at 100 EUR. 


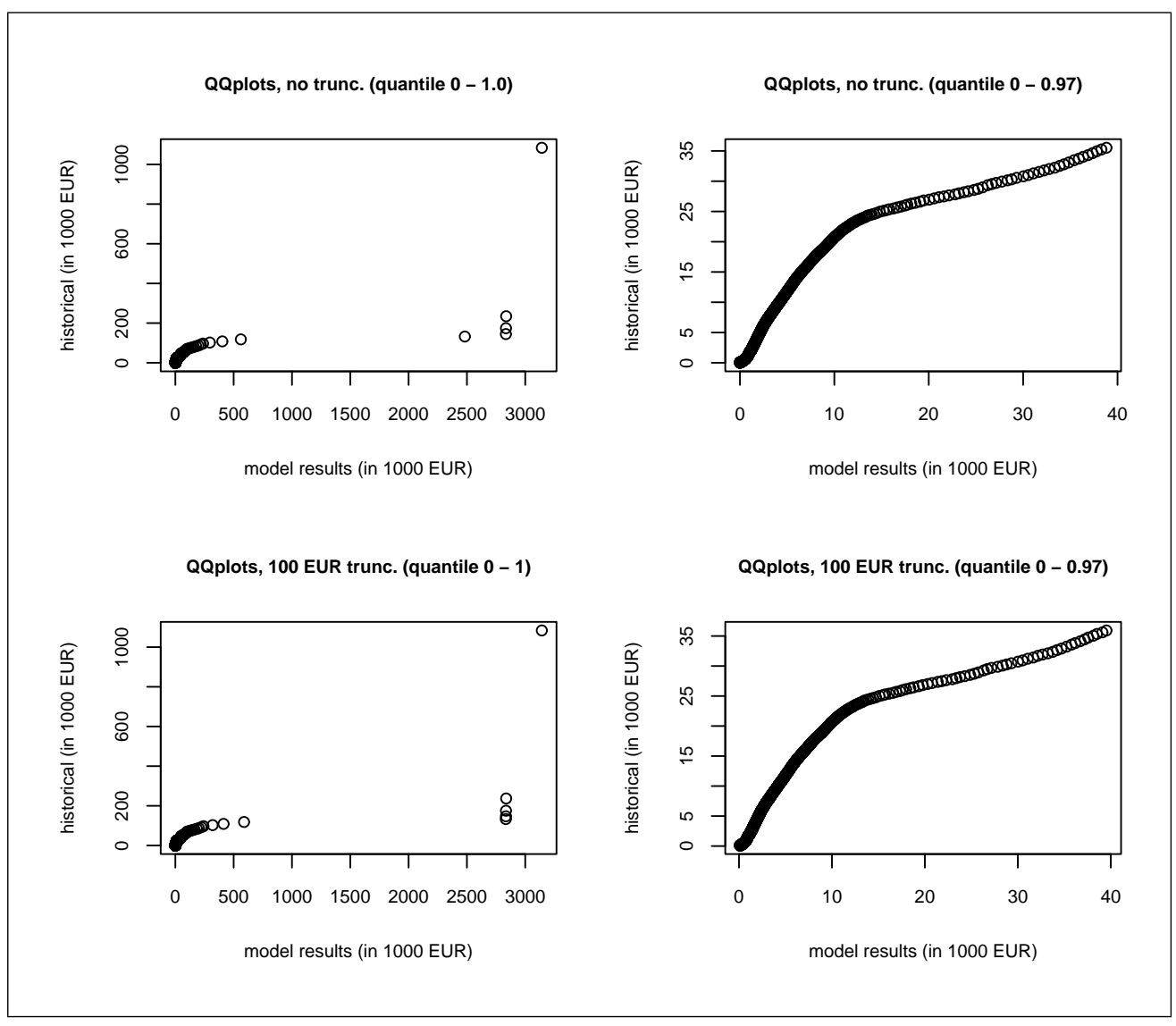

Figure 6.8: Potential loss - qq plots I (model versus historical data) 


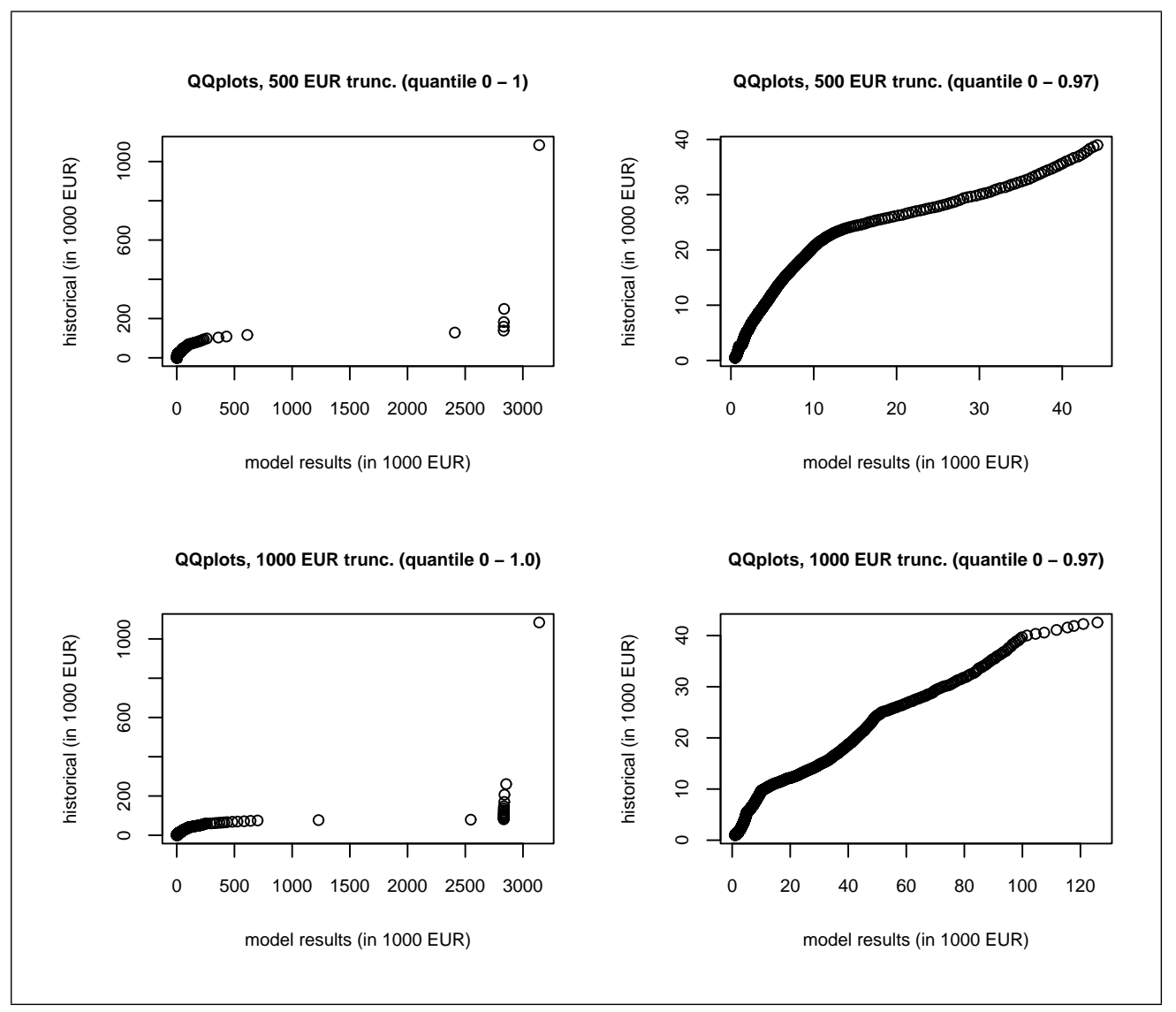

Figure 6.9: Potential loss - qq plots II (model versus historical data)

Two clear observations can be made from the comparisons made so far. The first is that the model emphasises (unexpected) high loss severity as compared to the historic losses that emphasises expected (low) losses. The second observation is that the body of the two distributions (quantile 0 - 0.97) compares favourably. Three reasons can be adduced for the first observation. Firstly, the model's initial loss distribution before aggregation is a "universe" of all losses possible. Secondly, the model's frequency of failure also includes forward-looking scenarios which might not have been experienced before and lastly, the experts might have been more pessimistic and given high probabilities to extreme scenarios.

We conclude that the combination of the favourable comparison of the body of the two distributions and the emphasis of the model on extreme losses (fat tail) validates the model especially for operational risk management, which is more concerned with high unexpected losses. Expected losses is always considered to be the cost of doing business. The next sections describe how the model is used 
to manage operational risk in FX and MM settlement.

\subsection{Managing Operational Risk with the Model}

The BN developed, like all other BNs propagate information and evidence in both forward and backward direction. This interesting feature enables our network to be used for both predictions and interventions. Scenario and causal analyses, and sensitivity analysis are the basis of managing operational risk with the FX and MM network. With scenario analysis, the value of any variable(s) can be set, the probabilities are then updated and the results of predictions are immediately known. Management can also simulate what can happen if controls are always in place, not in place or working inefficiently. The probable causes of losses can also be determined for interventions to be put in place.

Sensitivity analysis refers to analysing how sensitive the conclusions (probabilities of the hypothesis variables) are to minor changes. These changes may be variations of the parameters of the model or may be changes of the evidence also known as sensitivity to evidence - how sensitive is the conclusion to small changes in the evidence (Jensen, 2001).

Sensitivity analysis is performed, in our case, by making changes to the evidence and observing the target node. The target nodes in question are the numberof-failures and severity-of-losses for the frequency and loss severity networks respectively. Changes to evidence are made successively from node to node and each time observing how sensitive the target node is to these changes. The nodes nodes quality-of-SI and days delay were uncovered as the most sensitive nodes for the frequency and severity networks respectively.

The results from sensitivity analysis, help management to prioritize monitoring activities. Sensitivity analysis can also provide an assessment of which management options will have the greatest impact on the target nodes. One specific application of sensitivity analysis is the setting up of trigger levels with the initial state of the network for a specific KRI. When the KRI is triggered questions like "what actions should be taken and how effective can the actions be?" can all be answered with the Bayesian network. As new evidence are gathered (historically) new sensitivity testing should be run to determine the next important factors to monitor.

The separation of the frequency of failure network from the severity of losses network in the FX and MM BNs makes it possible for us to manage the drivers 
of frequency of failures and the drivers of loss severity separately. The results of predictions or interventions from the two sub nets can subsequently be aggregated to obtain a potential loss value. The following sections give some examples on how these are realized. These illustrations are not exhaustive but indicative of how the network can be used to manage operational risk.

\subsubsection{Managing the Drivers of Loss Severity}

In this section, an illustration is given on how the model can be used to manage the severity of losses. We commence with simple illustrations and proceeds to more complex illustrations.

\section{Illustration SI}

We are able to find out the greatest amount that can possibly be lost for each currency with the corresponding probability of occurrence. This is simply done by compiling the model, entering the evidence for the respective currency, and propagating the information through the network. For example, the largest amounts we can lose for USD, EUR and JPY are 250,000 - 500,000, 750,000 - 1,000,000, and less than 1000 EUR respectively. The corresponding probabilities of occurrence in percentages are 0.003, 0.019, and 1.0 respectively (see Figure 6.10). Such information will help the business line manager to know which currency to pay particular attention to. In this scenario EUR should receive the greatest attention and JPY the least. 


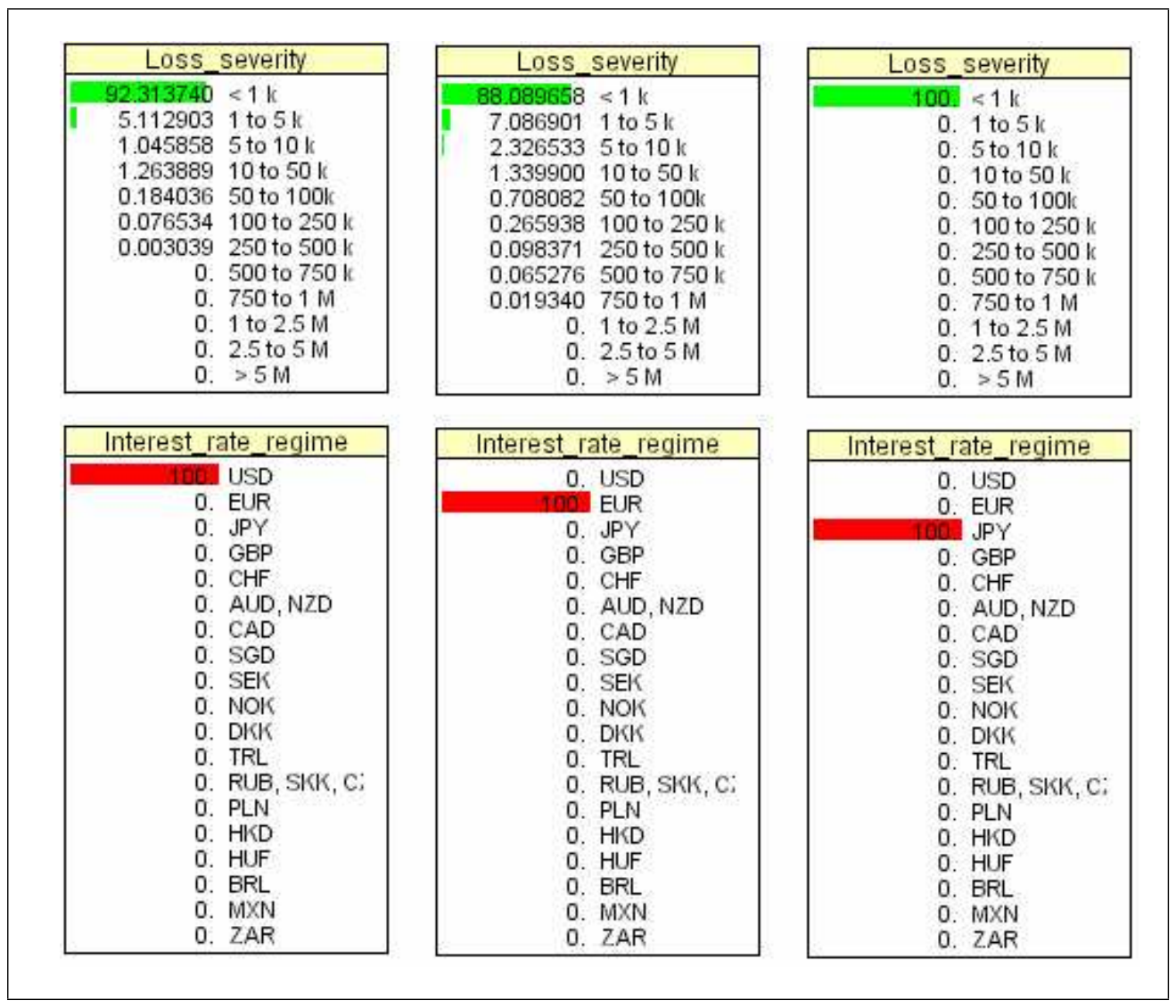

Figure 6.10: Illustration SI - largest loss amount

Addditionally, we can also find the loss distribtion for each currency under the various scenarios of days delay. This is done by instantiating (entering evidence) the desired currency and also instantiating one of the states of "days delay" and propagating the information through the network. This can be done for all the five states of days delay. Figure 6.11 gives the loss distribution for USD with the days delay state instantiated at three days. 


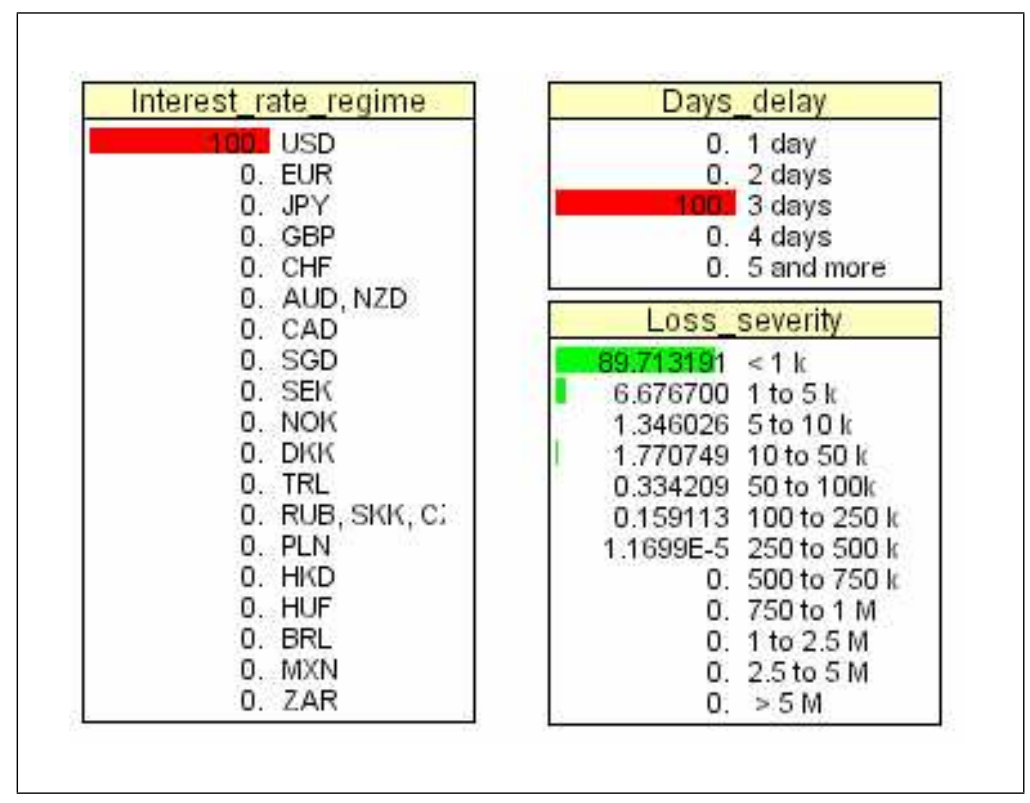

Figure 6.11: Illustration SI - loss with days delay

\section{Illustration SII}

In this illustration we create a scenario that the business line manager does not want to experience a single loss event (stop loss) of value more than 100,000 EUR and ask the question "which variables should be monitored?" To realize this, we instantiate the state "100 - 250k" of the loss severity node and propagate the information. Reading the probabilities from the first picture in Figure 6.12 show that it is likely to be an EUR trade (0.57) and should fail for either 3 days (0.38) or 5 or more days (0.36). The possible trade value for such a trade are $5-10$ million EUR with probability 0.19, 100 - 500 million EUR with probability 0.312 and $500-2000$ million EUR with probability 0.389. Clearly, there is no unique most likely combination of states for this scenario.

In order to obtain one unique combination of states being the most likely, we rather do belief updating with Most Probable Explanation (MPE) instead of the normal propagation. MPE simply means there is no other explanation that has a higher probability of occurring than that computed (see Section 3.3). In the Hugins modeling shell being used, this is done through maximum propagating the evidence. In each node a state having a value 100.00 belongs to the most likely combination of states (see second picture in Figure 6.12. The most likely combination of states for this case gives the trade as an EUR trade, with amount of 5-10 million EUR, and days delay of 5 or more days. The managerial implica- 
tion is that if we want to avoid a single loss of more than 100,000 EUR, we should monitor EUR trades of value more than 5 million and put in place mechanisms so that these trades do not remain unsettled for 5 or more days.

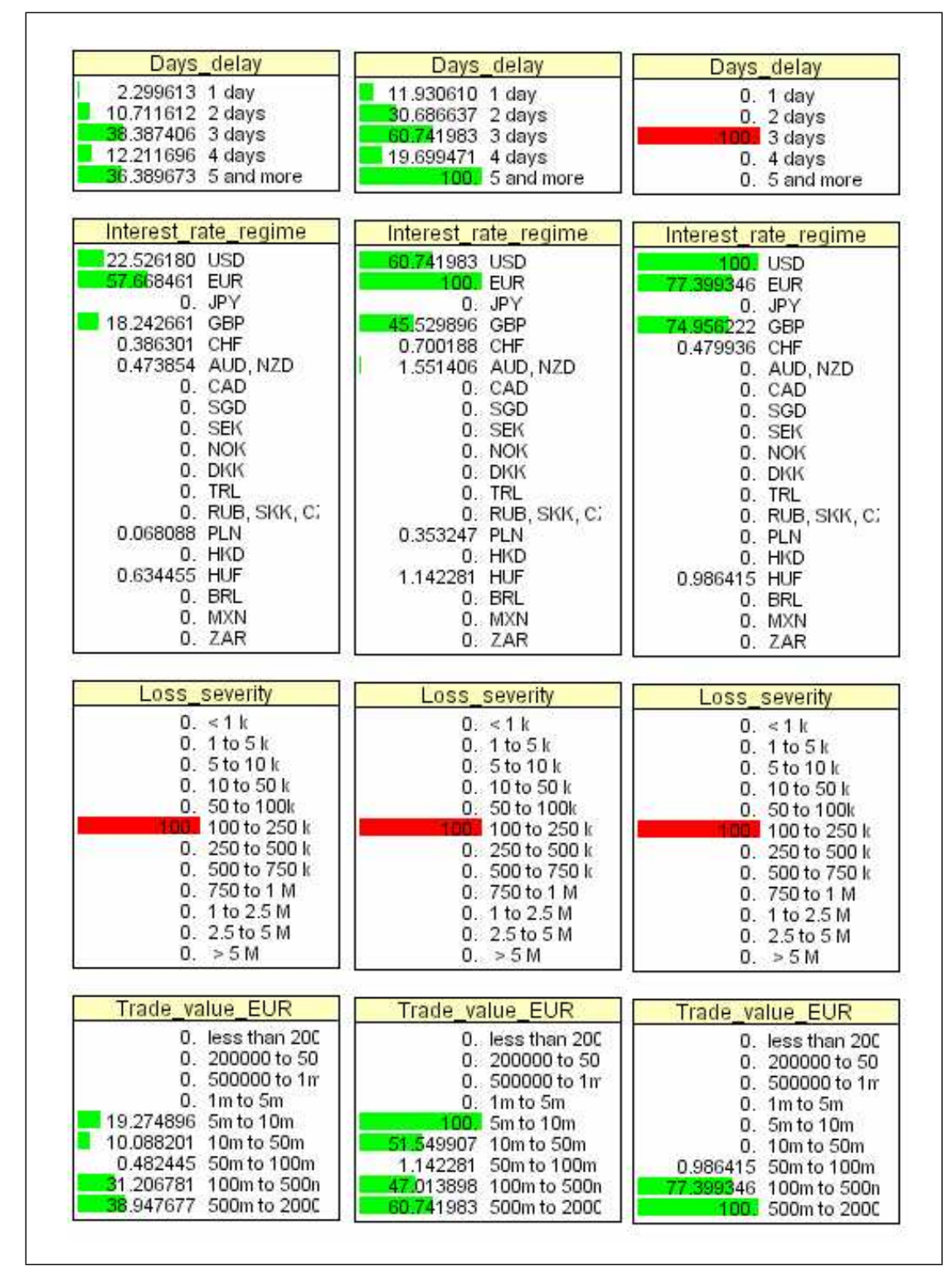

Figure 6.12: Illustration SII - stop loss with most likely combination of states

Monitoring EUR trades of value more than 5 million will mean the business manager will have to monitor almost 10 percent of all the EUR trades. This is not practical since a typical week has about 15,000 EUR trades in number! A 5 
day delay as obtained in our analysis is rather unlikely for high trade values. So we modify our scenario and say the most realistic worst days delay is three days. This is implies for example, that trades fail to settle on Friday but are re-settled on Monday. The three days failure information is incorporated by instantiating the state 3 days of the the node days delay.

Having done this, we maximum propagate the information through the network. The results suggest that we monitor USD trades more than 500 million EUR since these have the highest probability. A good risk management practice would however be to monitor USD, EUR and GBP trades with values more than 100 million EUR since all these three currencies with values above 100 million EUR have high probabilities of producing the target loss as shown in the last picture from the left in Figure 6.12.

\section{Illustration SIII}

This illustration is based on sensitivity analysis, which uncovered days delay as the most influential to the target node, loss severity. We create the following scenario: A mechanism is put in place to ensure that trades of value 50 million EUR or more are not allowed to fail for more than 1 day. We then want to investigate the affect on this mechanism on the loss distribution.

First, we need to revise the input probabilities (Conditional Probability Table) for days delay to reflect this scenario before compiling the network. We could simply input the probability value zero for trades more than 50 million EUR with days delay more than 1 day. This will produce a step function. We however adopt a more realistic approach that uses a function that produces a smooth curve. This function ensures that for days delay equals to one day, the probabilities increase with an increase in the trade value above 50 million EUR. For days delay more than one day the probabilities rather decreases with an increase in trade value above 50 million EUR. The function is given as follows:

Let the resulting probability of a delay of $k$ days, given the monetary value $V$ be of the form

$$
\pi_{k}(V)=\left\{\begin{array}{cc}
\alpha_{k} & \text { for } \quad V \leq V t \\
\frac{e^{\alpha_{k}+\beta(V-V t)}}{1+e^{\alpha_{k}+\beta_{k}(V-V t)}} & \text { for } \quad V \geq V t
\end{array}\right.
$$

where $V t$ is the monetary value above which we restrict the days delay.

This function is very closely related to a logistic regression and has the required 
property that

$$
\lim _{V \rightarrow \infty} \pi_{k}(V)=0 \text { for all } \mathrm{k} \geq 2
$$

The parameter value $\alpha_{k}$ is elicited and $\beta_{k}$ can be selected to reflect the anticipated change in the probabilities $\pi_{k}(V)$. (See Figure 6.13).

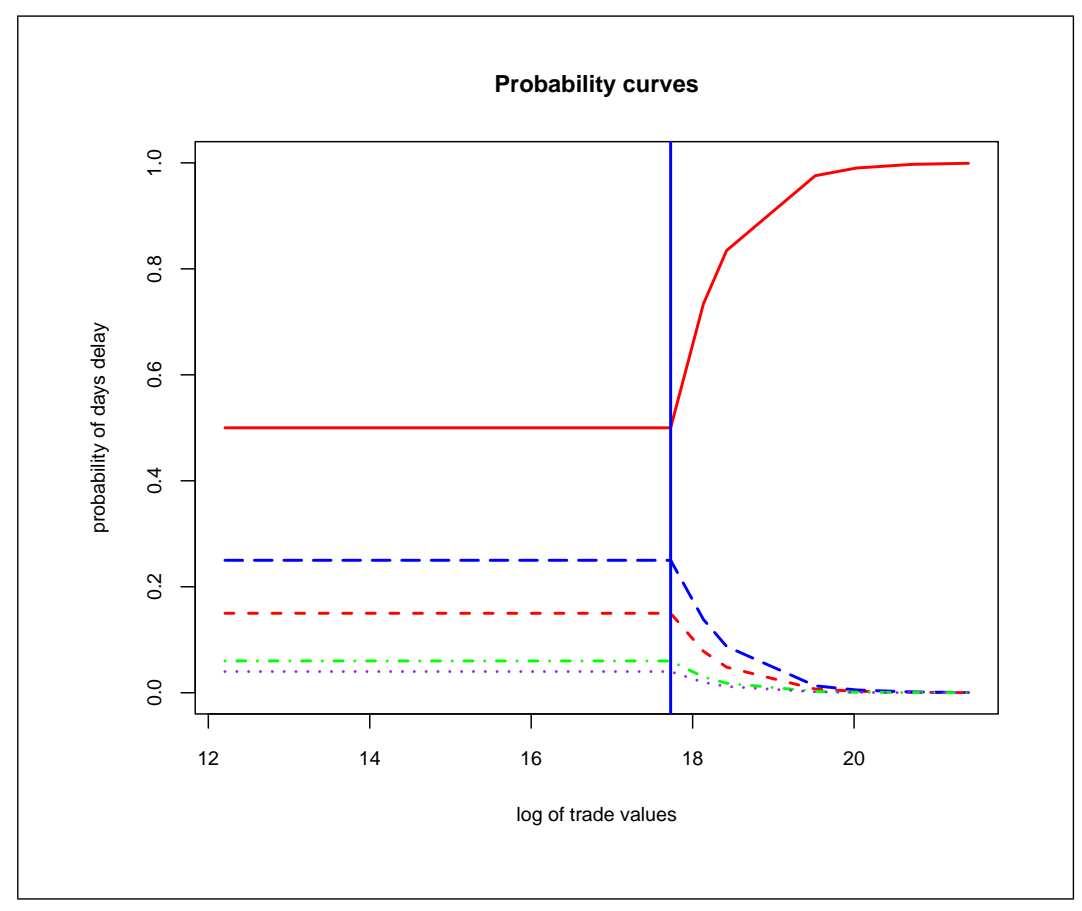

Figure 6.13: Probability curves for various days delays (daysdelay $=1$ is solid line, 2 is dash, 3 is longdash, 4 is dotdash and 5 is dotted). Vertical line is the $50 \mathrm{~m}$ EUR trade value

Re-compiling the loss severity network with this new set of probabilities (see appendix B) produces the scenario results (right picture) compared with the initial (baseline) probabilities in Figure 6.14 below. Notice the significant changes in the tails of the distribution. For a loss value of between 750,000 - 1 million EUR, our scenario has a reduced probability of occurrence by a factor of about 100 ( 5 in 100,000 as against 500 in 100,000). For a loss value between 500,000 - 750,000 EUR the probability is reduced by more than one-half ( 8 in 1000 as against 18 in a 1000). These are very interesting observations for operational risk, where our focus is on managing the tails of the loss distribution. 


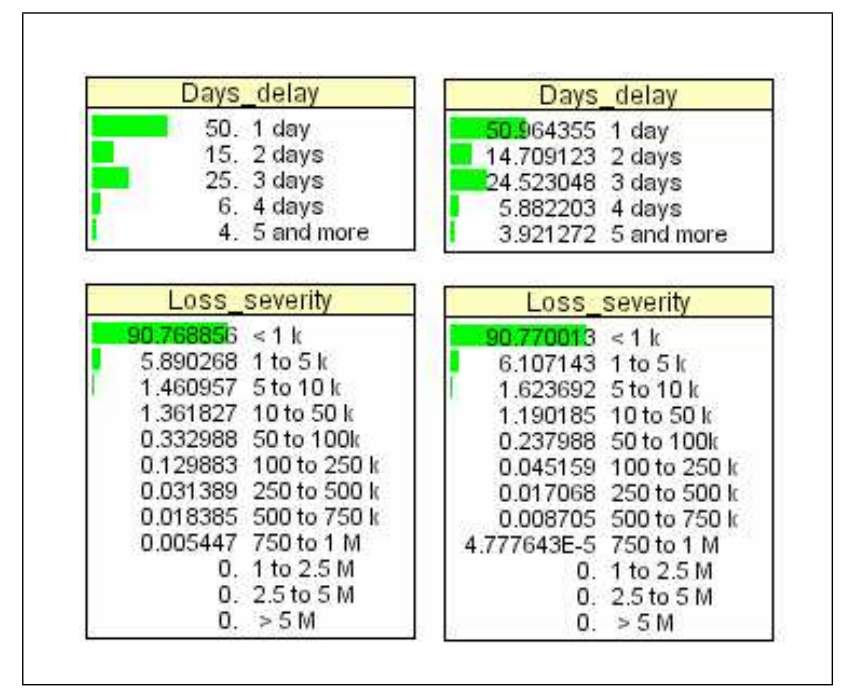

Figure 6.14: Illustration SIII - days delay scenario

\subsubsection{Managing the Drivers of Frequency of Failures}

Three illustrative examples are provided on how the model can be used to manage the drivers of frequency of failures. As already mentioned, the Bayesian network has two purposes: 1) managing OR in FX settlement and 2) calculating Economic Capital for internal purposes. For managing the drivers of frequency of failure, we first replace the terminal node of the frequency network, which is discrete with a continuous node. The simple reason is that with a continuous node, we gain a single overview of the number of failures, represented by its mean and variance instead of the discrete node that gives bar chart results. The added advantage is that we are able to set trigger levels by simply entering evidence into the continuous number-of-failures node. Entering evidence is done by altering the mean and variance of the continuous node. The integrity of the nework is however, still maintained in this adjustment.

The continuous node has to be quantified with a mean and variance. The initial assumption made that the elicited number of failures follows a Poisson distribution is still maintained. We therefore use the typical number of failures elicited as the mean and variance for the continuous node. The illustrative examples follow:

\section{Illustration FI}

This example concentrates on the front and back office systems in place. Here, we seek to find the effect the node backoffice-system-downtime has on the number 
of failures and also the effect an increase in trade volume has on the front office system and trade capture.

To see the effect of a backoffice system failure, the model is first run in its initial state and the relevant outputs (number of failures and paymentsystem-inputmethod) are noted. We instantiate the node backoffice-system-slow-downtime to the state more than 30 mins and the node backoffice-system-failtime to the state critical. This set of information is propagated through the network and the new set of results are also noted. Both results are shown in Figure 6.15. The probability of a manual input into the payment system increases from $4 \%$ to $70 \%$ and the subsequent mean and variance of the number of Failures increase respectively from $(2.3,3.0)$ to $(3.0,4.0)$. The effect of a back office system failure on the number of failures does not appear to be substantial.

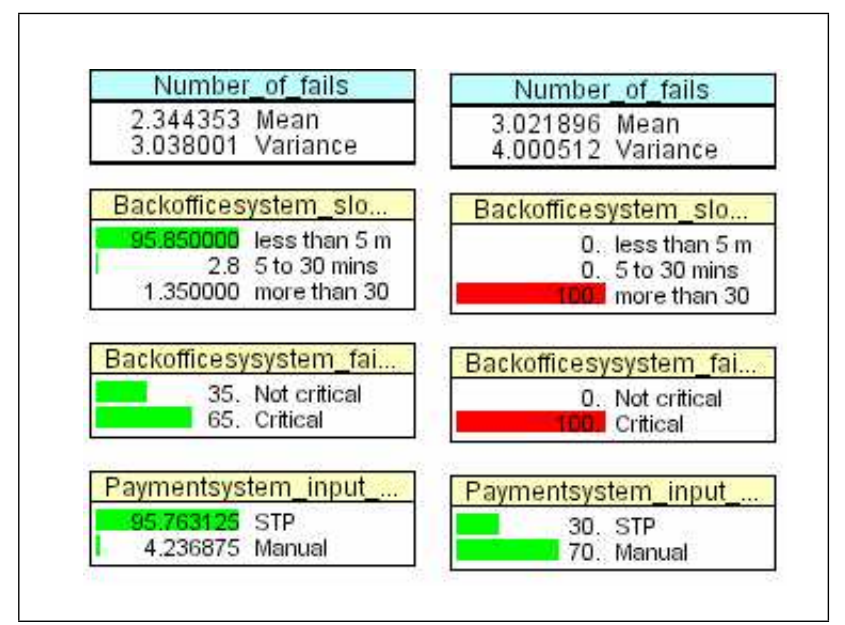

Figure 6.15: Illustration FI - back office system failure

The effect of an increase in trade volume on the front office system and trade capture can also be investigated. Going through the same process but this time instantiating the node trade volume to the state 100,000 - 150,000 the results in Figure 6.16 is obtained. The output shows that the probability of the front office system malfunctioning or not available doubles and that for incorrect or untimely trade capture triples. The managerial implication is that, once the volume of trades increase above its usual operating level, the front office system should be monitored. Trade capturing acitivities like inputing of trade details should be done carefully since the propensity to capture trades incorrectly or in an untimely manner increases. The increase of risk is also significant (double) at 
a trade volume level of between 40,000 to 100,000 (not shown).

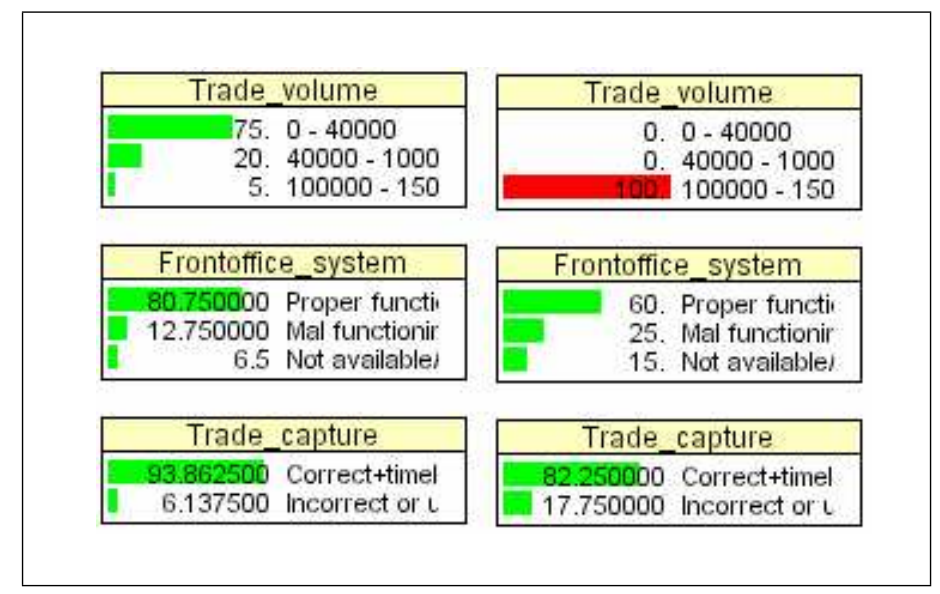

Figure 6.16: Illustration FI - effect of trade volume on systems

\section{Illustration FII}

The illustrations in this section is about causal analysis and concentrates on the part of the network that deals with back office processing. We specifically examine issues involving confirmation matching and the quality of standing settlement instructions (SI).

We first examine the likely causes of trades being highly vulnerable to failure. Before proceeding any further, we remark that the network is designed such that back office process confirmation matching is considered as a control of the front office processes. That is, should there be an incorrect trade, back office is expected to detect it before the trade settles. Going back to our scenario, the state high of the node vulnerability-to-failure is instantiated. We assume we know that the front office system is functioning properly. This evidence is included by also instantiating the state proper functioning of the node front-office-system.

The output of this scenario is shown in Figure 6.17. The most probable explanation (by normal propagation) is that confirmation matching is not being done and trades are either captured incorrectly or in an untimely manner. Note also the significant change in the probabilities of the node confirmation matching. For example, probability for the state done incorrectly increases from $2 \%$ to $10 \%$. What this means is that for a typical trading week, where the front office system is functioning properly, trades will have a high vulnerability to fail if confirmation matching is done only $40 \%$ of the time. 


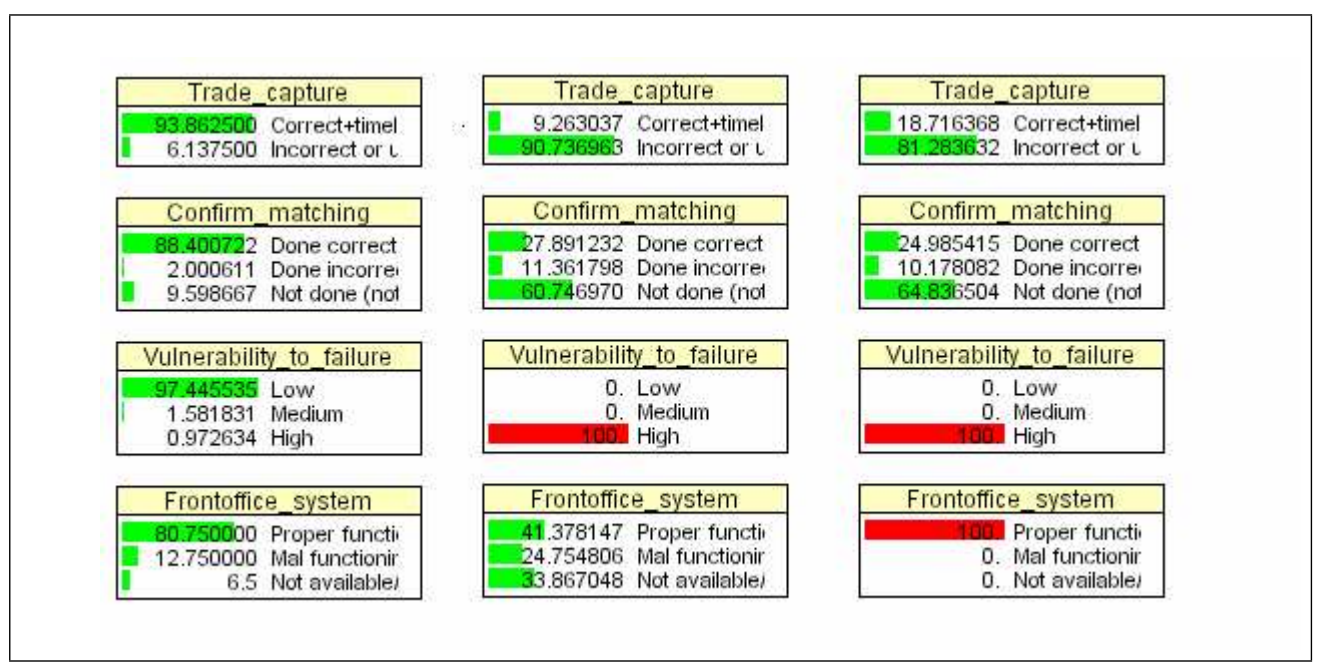

Figure 6.17: Illustration FII - causes of vulnerability to failure

The use of Standing Settlement Instructions (SI) is very important in FX and MM settlement. Counterparties to trades do change their SI occassionally and are required to announce to all parties the changes that have been made. Back office is supposed to ensure that the SIs in place are current and accurate. We investigate the effect of the quality of the SIs in place on the number of failures. This is done by instantiating the node Quality-of-SI and observing the effects at the node number-of-failures. The results as shown in Figure 6.18 indicates that the number of failures is very sensitive to the quality of SI. The mean and variance of number of failures increase from $(2,2.3)$ of the initialized state to $(4.0,5.5)$ when the node Quality-of-SI is instantiated at the state bad. This is a clear indication that maintaining the quality of SI is a very important activity that should be constantly monitored.

\section{Illustration FIII}

There are two parts to this illustrative example. The first part seeks to find the most important nodes to monitor under the scenario that a zero number of failures is desired for a period of one week. The second part deals with the setting of trigger levels to help manage OR.

To determine the important nodes under the zero mean number of failures scenario, we run a sensitivity analysis to determine how sensitive our nodes are to changes made to the number of failures node. To do this the model is first com- 


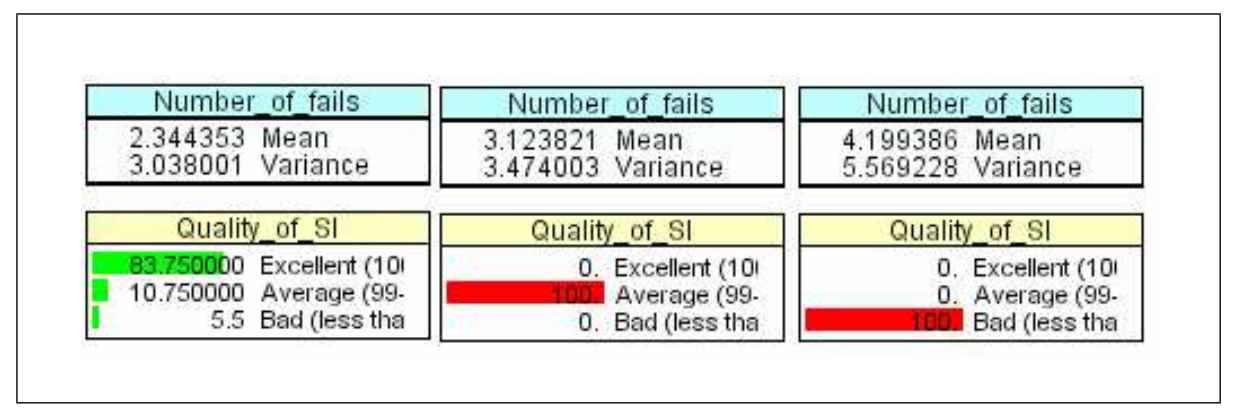

Figure 6.18: Illustration FII - effect of quality of SSI

piled in its initial state and the node probabilities are noted. We then enter the new evidence (mean number of failures is zero) into the network and propagate this information through the network. The results show (we present only the relevant nodes) that the most sensitive nodes in order of importance are Qualityof-SI, trade-capture, and confirmation-matching (see Figure 6.19). This kind of analyses provide a first hand assessment of which managerial options will have the greatest impact on the target node number-of-failures. In this case, management might want to prioritize efforts on these influential nodes in order of their importance.

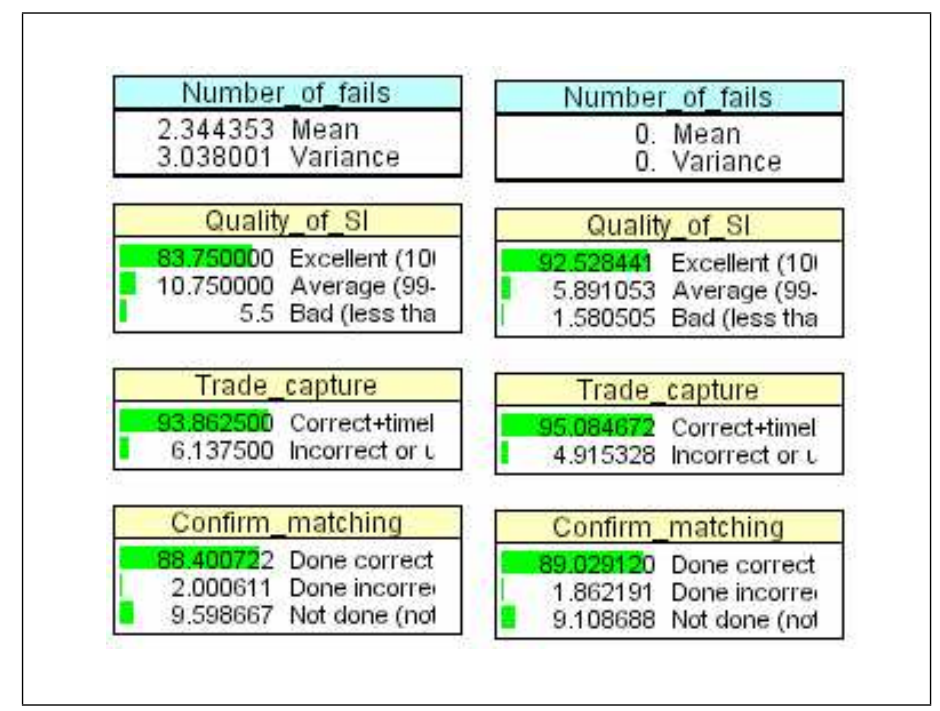

Figure 6.19: Illustration FIII - most sensitive nodes 
The Bayesian network can be used to set trigger levels, which when exceeded should prompt some action from management (Alexander, 2003). A trigger level is set with an initial state of a KRI. Before setting the trigger levels, we first create the scenario that management does not want the mean number of failures in a week to exceed 6 failures (mean +1 std). The illustration begins by first compiling the network in its initial state and noting particularly the value of the state not done of the node confirmation-matching to be $9 \%$. We proceed by entering the evidence (mean number of failures $=6$ ) and propagate this information through the network. The problem can further be isolated by setting the node paymentsystem-input-method to the state STP and the node front-office-system to the state proper functioning. This will ensure that we do not attribute the number of failures to system failures. After re-running the model, the results show that the value of the state not done of the node confirmation-matching has increased from $9 \%$ to $12 \%$ as shown in Figure 6.20.

We can now decide to use the state not done of the node confirmation-matching as the trigger KRI, with a trigger value of $12 \%$. The implication is that, if about $12 \%$ of the time, confirmation matching is not done (that is, not done, not sent or sent with no response) we risk experiencing a number of failures not less than 6. Whenever our chosen KRI (confirmation matching not done) exceeds 12 percent, this KRI is triggered and management would have to act. The possible actions management can take are clear from the probability Tables in Figure 6.20 - reassess the quality of SIs in place and confirm trades before they are settled! 


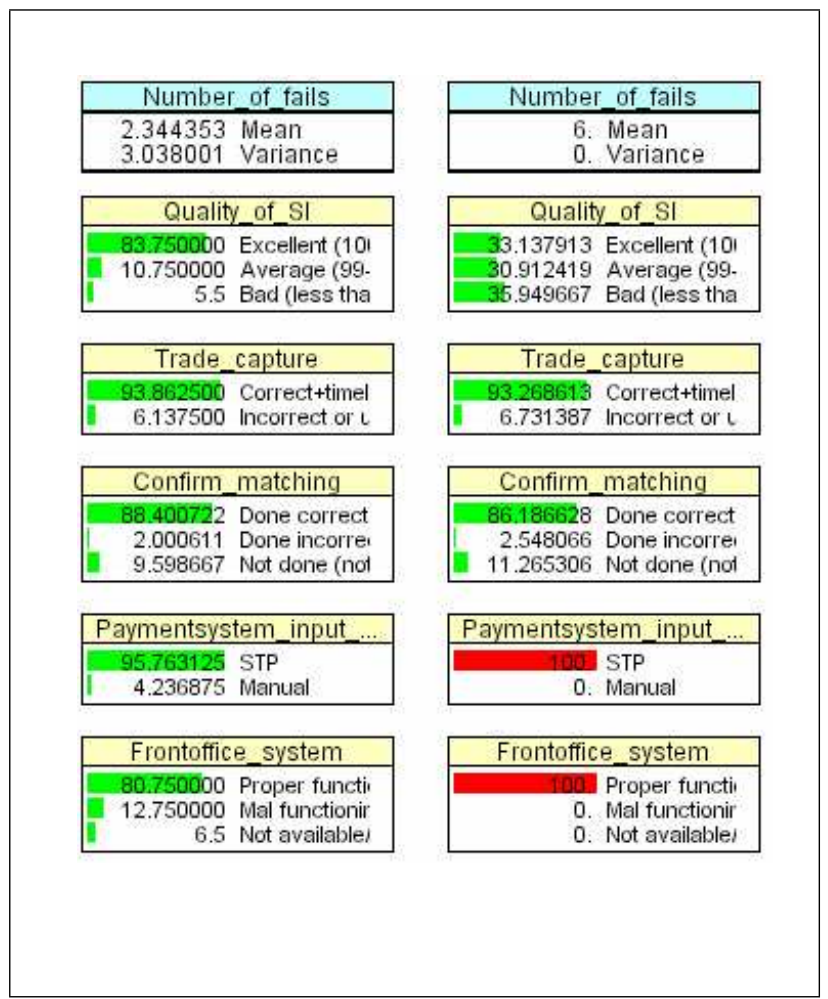

Figure 6.20: Illustration FIII - most sensitive nodes

\subsubsection{Prediction of Operational Value at Risk}

The effects of the various scenario analyses done on both the frequency and severity network can be aggregated or combined through Monte-Carlo simulations to obtain an operational Value at Risk number. The aggregation through MonteCarlo simulations is no different from that described earlier and will therefore not be repeated. Prediction of operational Value at Risk is important for management purposes since the potential loss under various interventions and scenarios can be obtained. These results can help management judge, for example, if interventions are necessary or not.

The output of illustration SIII for example, may be combined with the output from the frequency network in its initial state to obtain an operational VaR for the case where trade values of more than 50 million EUR are not allowed to fail for more than 1 day. The potential loss due to an increase in trade volume and also the quality of Standing Settlement Instructions can all be investigated by combining their respective illustrations (FI and FII) with outputs from the initial 
state of the loss severity network. Other combinations are possible depending on the desired objective. 


\section{Chapter 7}

\section{Summary and Conclusions}

\subsection{Summary of Work}

This work has argued that the current emphasis of operational risk management is expected to shift from the macro (top) level to the micro (business unit) level where operational risk losses are actually experienced. This anticipated shift in emphasis will require models that can be used for managing the details of a desired process. Of the present models available for managing operational risk, Bayesian networks stands out as the model of choice.

The usefulness of Bayesian networks for managing operational risk at the business unit level has been demonstrated with a real-world case study. Through the application of a BN to a bank's Interbank Foreign Exchange settlement process, the case study has been able to model the causal relationships among the operational risk attributes, such as causal factors, Key Risk Indicators, frequency of settlement failure and the loss given settlement failure. The Bayesian network developed showed the several levels of dependencies among the risk factors. This allowed the model to be used for a detailed analysis of the risk and causal factors within the settlement process.

Results from our Bayesian model compared well with historical data for quantile values from 0 to 0.97 . Above this value, the model showed higher losses than the historical data. The significant difference in the tails of the two distributions was expected since our model included some scenarios which has not been experienced in the past. Using the model as a management tool, we have adequately demonstrated, with several real-world illustrations the day-to-day management of operational risk within the process. In addition, practical guidance on how 
to implement an operational risk Bayesian network from start to finish for a desired process has also been provided in the study. The essential elements of this guidelines are summarised as follows:

1. Establish the domain problem as well as the specific objectives for the BN. The domain problem could be for example, FX settlement process and the objectives may be managing a specific operational risk attribute(s) within the process;

2. Conduct a risk and process mapping for the business process being modeled. The risk and process mapping has two advantages: 1) it gives a better understanding of the process being modeled and 2) it uncovers the causal or risk factors and KRIs relevant to the desired operational risk attribute(s) to be managed;

3. Construct the BN structure by causal or risk factors, KRIs and the operational risk attribute(s) with arcs to establish the causal relationships among these elements. This is an iterative process and should be done together with the domain experts, who will later validate the structure of the network. It must be emphasised that there is no unique BN structure to a particular process but a best fit given the modeler and experts. A trade-off should be made between detailed causal modeling and the practicallity of implementing the network. A very detailed BN may be desirable for managing the fine details of operational risk but could be difficult to quantify and maintain;

4. Quantify the BN. Quantification can be done with either subjective, objective data or a combination of subjective and objective data. The use of either subjective or objective data will depend on the particular process being modeled and the availability of data. This decision should also be guided by a decision on how the network will be validated for use;

5. Follow a well defined elicitation protocol when conducting probability elicitation to obtain subjective data. A well defined protocol will help anticipate and mitigate biases that might be present in the elicitation process. The protocol should also provide details on how the experts' estimates are validated. A BN is only as good as its prior probabilities. A suggestion is to elicit estimates from several experts (if available) and use bootstrap methods to aggregate the estimates (Meyer and Booker, 2001); 
6. Check the performance of the BN model before using it as an operational risk management tool. Some ways of doing this includes 1) making realistic comparisons with available historical data, 2) running plausible scenarios of the model and making realistic judgement on the scenario results, and 3) validating the model results with the internal audit department;

7. Maintain the network by making periodic adjustments to the sturcture of the network and the initial prior propabilities. The initial prior probabilities can also be updated when objective data becomes available. These adjustments are supposed to track changes in the business process and control environment. This ensures that the BN model is up to date and reflects reality at all times.

\subsection{Further Work}

In the elicitation of probability distribution, the usual approach is to assume, a priori, that the quantity to be elicited follows a certain prescribed distribution. Another approach may be to rather elicit some qantiles (for example, 5\%, 25\%, $50 \%, 75 \%$ and $95 \%$ ) values of the estimate and then fit the best distribution to the estimates. That is, the problem is translated into that of model selection, where we use some model selection criteria to find the best fitting model to the data. This approach needs to be further investigated.

Operational risk loss distributions have fat tails. In practice certain known distributions (for example, lognormal) are fitted to the loss data before aggregating with the frequency distribution to obtain the aggregated potential loss distribution. This approach together with kernel density estimation methods was used in the case study. The use of kernel density methods with adaptive bandwidth selection was able to capture the tails of the distribution reasonably well. Further investigations with several different data sets are needed to assess the performance of using kernel density estimation methods in capturing the tails of operational risk loss data. A comparison of this approach with the parametric distributions used in practice could also be investigated. Investigating the effect of this approach on the aggregated potential loss distribution would be of interest.

It was suggested during the elicitation process that several experts, if available, should be used to estimate the desired quantity and their estimates aggregated through bootstrap methods. This approach is in contrast to the present practice in industry where there is an assessor and a superior to this assessor who evaluates the estimate given by the assessor. Although, the merit of the former approach 
is obvious, a comparative analysis of the two approaches should be carried out in the context of operational risk.

In managing operational risk with a $\mathrm{BN}$, one may be interested in estimating the cost and benefits of certain actions and interventions. Utility and decision nodes are needed in such situations. An investigation in extending the usefulness of the $\mathrm{BN}$ by incorporating utility and decision nodes in a real-world application would be useful. A BN with decision and utility nodes is called an Influence diagram.

\subsection{Conclusion}

We conclude by maintaining the initial assertion that Bayesian networks are potentially powerful tools for managing operational risk at the business unit level. The objective of this study has been to substantiate this contention. The present practice of managing operational risk with KRIs, risk drivers and control approaches can be extended further by combining the essential elements of these approaches into one Bayesian network structure. Such a Bayesian network structure has two advantages: 1) the ability to model the causal relationships among the risk drivers, KRIs and a desired operational risk attribute and 2) the ability to combine and make use of the advantages of the essential elements of the Advanced Measurement Approach (internal and external loss data, scenario analysis or expert judgement, business and risk control environment).

It is hoped that the practical guidance provided in this work will contribute to position Bayesian networks as the preferred tool for managing Operational Risk at the micro (business unit) level, particularly, the managing of operational risk within Foreign Exchange and Money Market settlement process, which has experience huge financial losses recently.

The thesis is also intended to challenge the view held by some operational risk practitioners that Bayesian networks are too complex to maintain, too difficult to construct and give little return for the effort put in. To be sure, the effort involved in the development of a Bayesian network is substantial. That is mainly owing to the lack of experience in dealing with the practical details of it's implementation. There is currently also a lack of guidance on how to overcome the practical problem. It is hoped that this thesis provides a contribution in this respect. Certainly there is a clear need to qunatify and manage Operational Risk and Bayesian networks provide a theoretically sound base to meet this need. 
Appendix A

Questionnaire 


\section{Introduction to Questionnaire}

This questionnaire is part of the on-going work to construct a model that can be used to manage; reduce the incidence and severity of operational risk within the FX settlement process of the bank. Because of your considerable knowledge and expertise in the FX settlement process within the bank, I would like you to participate in this risk assessment study of the foreign exchange settlement process.

Your role will consist of two parts: 1) giving your opinion on the rate of occurrence and magnitude of certain defined incidence within the causal network that has been built with substantial input from you and 2) reviewing or refining your input.

Your knowledge is of interest no matter how general or vague it is. You will probably be more knowledgeable about some characteristics than about others. Some of the questions concerns events that might have happened already whiles others concern potential events with no historical occurrence whatsoever. The scenarios given in the questions are supposed to help you estimate the likelihood of occurrence of some of these rare events.

It is important, however, that you be very careful in answering the questions. Think over each question carefully and if it is not clear to you, ask for further explanation. Also if at any time you wish to change a previous answer after further thought, fell free to do so. The questionnaire is designed to aid you in communicating your knowledge to the experimenter, so any comments regarding the clarity of the questions are most welcome.

On the question sheets, you are required to mark your answers on the response scale and if possible write a number next to your mark. In addition, some questions will also require you to give a numerical estimate of your answer in the boxes provided.

The quality of the model and its ability to be used to manage operational risk within the FX settlement process will depend very much on the accuracy of the answers you provide. The answers you provide will not under any circumstances be used to evaluate your performance at the Bank.

It is anticipated that the entire elicitation process will comprise six interviews of approximately two hours each over a period of 6 weeks starting in the third week of April. There are two networks (interbank, money market) and each has been divided into three coherent sections. Each of the two hour interviews will focus on one section of the two networks.

Elicitation processes are always subjected to some form of biases. These biases are explained on the next page.

Figure A.1: Questionnaire introduction 


\section{$\underline{\text { Bias }}$}

Bias is a deviation from reality or what we consider desirable. There are basically two kinds of bias that may be present within the elicitation process. These are motivational bias and cognitive bias.

1. Motivational bias is driven by our human needs for example social pressure, impression management or wishful thinking. A particular example may be giving estimates to impress supervisors.

2. Cognitive bias occurs when your estimates fail to follow normative statistical or logical rules due to the way human beings process information. Cognitive bias includes:

a. Inconsistency - when you become inconsistent in your reasoning. This may be for example, due to fatigue

b. Availability - when you forget certain details and are not able to provide them

c. Anchoring - when you start with a first impression and you stick to this impression and only later make minor adjustments.

Figure A.2: Questionaire introduction - bias

\section{Interview information:}

Please provide the following information

Date.

Time

Place. Duration

Expert's name

Job responsibility

Number of years you have worked at your present job.

Figure A.3: Questionnaire introduction - interview information 


\section{Trade volume}

Please read all three questions on this sheet before giving your answers.

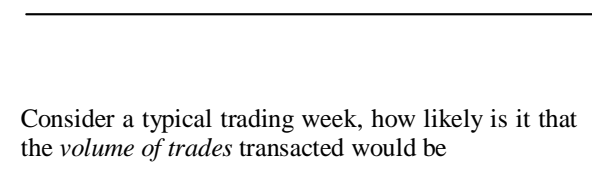

less than 40,000 trades?

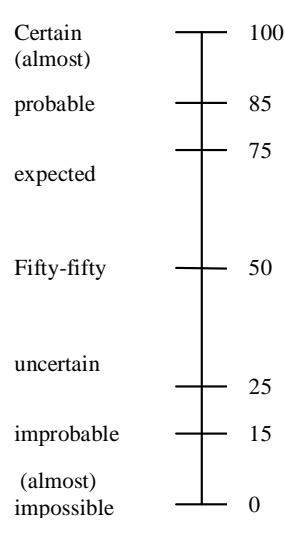

between 40,000 to 100,000 trades?

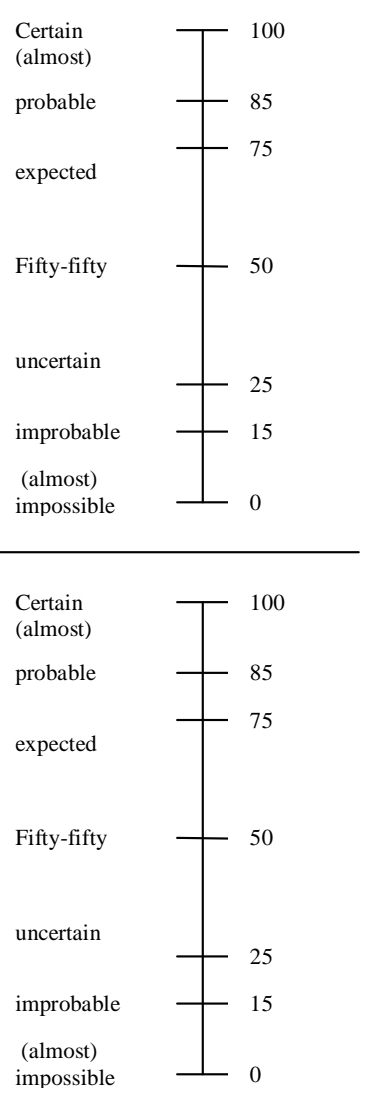

more than 100,000 trades? 


\section{Gross/Netting Settlement}

Please read through all the questions carefully before giving your answers.

Consider a particular day with a trade volume of about 8000 trades. What percentage (typical value and range)

of these trades will be

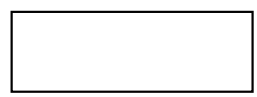

Settled Gross?

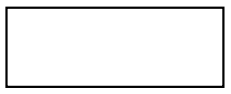

to
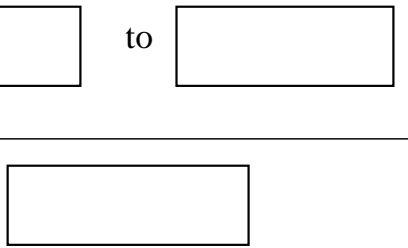

Settled on a Netting basis?

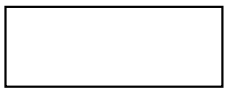

to

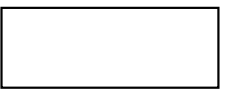

Figure A.5: Sample Questionnaire - gross or netting settlement 


\section{Days delay (1)}

This section consists of two sheets - days delay (1) and days delay (1). Please spread both Sheets on your table and read all five questions before giving your answers.

Consider a typical settlement day; a particular trade has failed to settle correctly. How likely is it that the trade will remain unsettled after

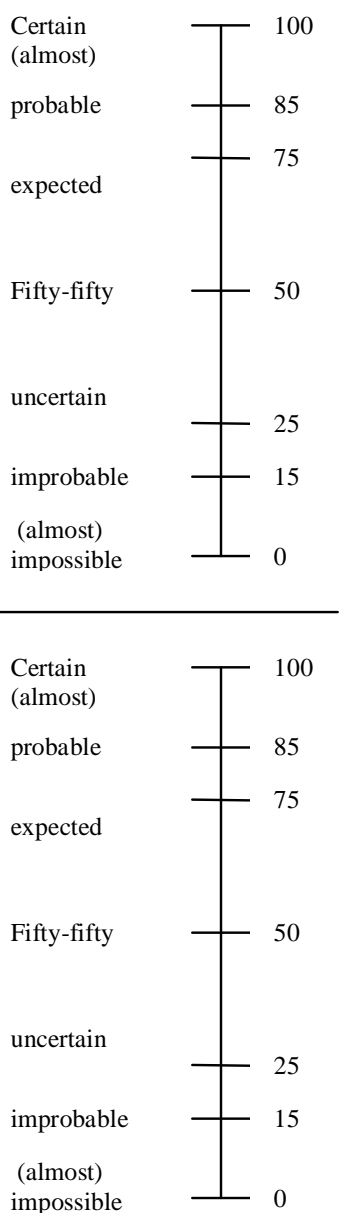

2 days

1 day

Figure A.6: Sample Questionnaire - days delay (1) 


\section{Days delay (2)}

This section consists of two sheets - days delay (1) and days delay (2). Please spread both sheets on your table and read all five questions before giving your answers. failed to settle correctly. How likely is it that the trade will remain unsettled after

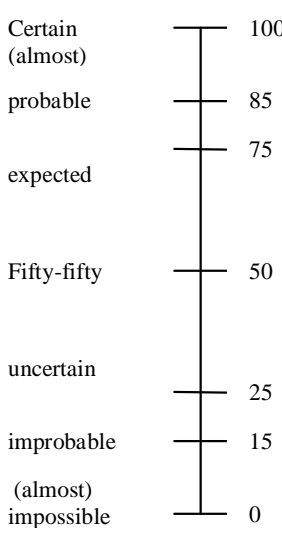

3 days

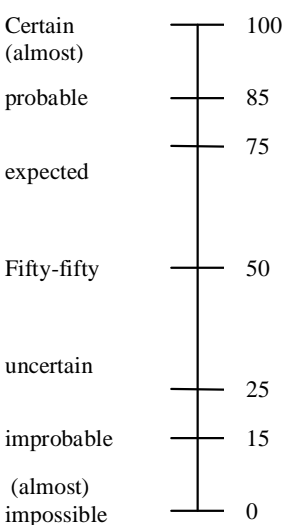

4 days

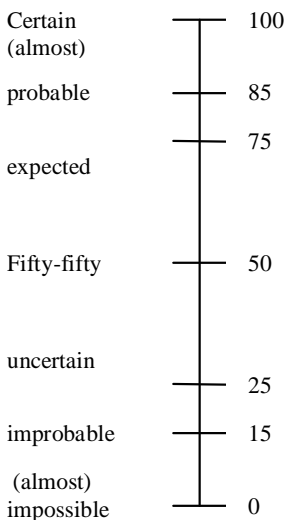

Figure A.7: Sample Questionnaire - days delay (2) 


\section{Confirmation Matching}

Please fill in the probability tables with your answers. The probability tables should be intepreted as shown in example $(*)$ below. The response scale is to guide you estimate your probabilities.

Example *

Consider a typical day where a particular trade of type a) Standard (Spot/Fwd/Swap) or b) Non Stand/Struct, NDF or Options is to settle a) Gross or b) Netting. This particular trade has a) Standing Settlement Instructions (SSI) in place or b) No SSI in place .

How likely is it that confirmation matching for this particular trade under the above scenarios will a) be done correctly (matched), b) done incorrectly, and c) not be done (not sent, no feedback etc)?
Response scale

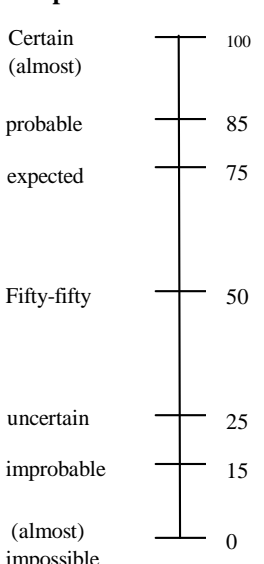$$
\text { (1) }
$$

\begin{tabular}{|l|c|l|l|l|}
\hline SSI in place & \multicolumn{3}{|c|}{ Yes } \\
\hline Gross/Netting settle & \multicolumn{2}{|c|}{ Gross settlement } & \multicolumn{1}{c|}{ Netting settlement } \\
\hline & $\begin{array}{l}\text { Standard } \\
\text { (Spot/Fwd/Swaps) }\end{array}$ & $\begin{array}{l}\text { Non Stand/Struct (NDF, } \\
\text { Options, etc) }\end{array}$ & $\begin{array}{l}\text { Standard } \\
\text { (Spot/Fwd/Swaps) })\end{array}$ & $\begin{array}{l}\text { Non } \\
\text { Stand/Struct(NDF } \\
\text { Options, etc) }\end{array}$ \\
\hline Trade Type & & & & \\
\hline Done correctly & & & & \\
\hline Done incorrectly & & & & $100 \%$ \\
\hline Not done & $100 \%$ & $100 \%$ & $100 \%$ & \\
\hline Total & & & & \\
\hline
\end{tabular}

\begin{tabular}{|l|c|l|l|l|}
\hline SSI in place & \multicolumn{3}{|c|}{ No } \\
\hline Gross/Netting settle & \multicolumn{2}{|c|}{ Gross settlement } & \multicolumn{2}{c|}{ Netting settlement } \\
\hline Trade Type & $\begin{array}{l}\text { Standard } \\
\text { (Spot/Fwd/Swaps) }\end{array}$ & $\begin{array}{l}\text { Non Stand/Struct(NDF, } \\
\text { Options, } \text { etc) }\end{array}$ & $\begin{array}{l}\text { Standard } \\
\text { (Spot/Fwd/Swaps) }\end{array}$ & $\begin{array}{l}\text { Non } \\
\text { Stand/Struct(NDF } \\
\text { Options, } \text { etc })\end{array}$ \\
\hline Done correctly & & & & \\
\hline Done incorrectly & & & & \\
\hline Not done & & & & \\
\hline Total & $100 \%$ & $100 \%$ & $100 \%$ & $100 \%$ \\
\hline
\end{tabular}

Table A.1: Matrix format questionnaire - Confirmation matching 


\section{Number of settlement failure}

$\underline{\text { Meaning of terms. }}$

1. The term "Typical value" means the most likely or most frequent number.

2. The term "range" can be interpreted as two values (5th and 95th percentile) around your estimate (typical value) such that you are confident $90 \%$ of the time that it covers the typical value.

The matrix table should be interpreted as given in the example below.

Example

Consider a typical working week with a trade volume of a) less than 40,000, b) 40,000 to 100,000 trades, and c) more than 100,000 trades. The vulnerability of failure of a trade is either a) Low, b) Medium or c) High. Consider in addition the situation where the quality (ratio of \# of correct to total \# ) of Settlement Instructions (SI) is either a) Excellent (100\%), or b) Fair or c) Poor.

What is the typical number of trades you will expect to fail under the above scenarios? Please give a range of values for your estimate.

\begin{tabular}{|c|c|c|c|c|c|c|}
\hline Quality of SI & \multicolumn{6}{|c|}{ Excellent (100\%) } \\
\hline Vulnerability to Failure & \multicolumn{3}{|c|}{ Low } & \multicolumn{3}{|c|}{ Medium } \\
\hline Trade Volume (weekly) & $<40,000$ & $40,000-100,000$ & $>100,000$ & $<40,000$ & $40,000-100,000$ & $>100,000$ \\
\hline Typical \# of failures & & & & & & \\
\hline Range & & & & & & \\
\hline
\end{tabular}

\begin{tabular}{|c|c|c|c|}
\hline Quality of SI & \multicolumn{3}{|c|}{ Excellent (100\%) } \\
\hline Vulnerability to Failure & \multicolumn{3}{|c|}{ High } \\
\hline Trade Volume (weekly) & $<40,000$ & $40,000-100,000$ & $>100,000$ \\
\hline Typical \# of failures & & & \\
\hline Range & & & \\
\hline
\end{tabular}

\begin{tabular}{|c|c|c|c|c|c|c|}
\hline Quality of SI & \multicolumn{6}{|c|}{ Fair $(98-100 \%)$} \\
\hline Vulnerability to Failure & \multicolumn{3}{|c|}{ Low } & \multicolumn{3}{|c|}{ Medium } \\
\hline Trade Volume (weekly) & $<40,000$ & 40,000 to 100,000 & $>100,000$ & $<40,000$ & 40,000 to 100,000 & $>100,000$ \\
\hline Typical \# of failures & & & & & & \\
\hline Range & & & & & & \\
\hline
\end{tabular}

\begin{tabular}{|l|l|l|l|l|l|l|}
\hline Quality of SI & \multicolumn{5}{|c|}{ High } \\
\hline Vulnerability to Failure & \multicolumn{2}{|c|}{$<40,000$} & \multicolumn{2}{|c|}{40,000 to 100,000} & \multicolumn{2}{|c|}{$>100,000$} \\
\hline Trade Volume (weekly) & \multicolumn{2}{|c|}{} & & & \multicolumn{2}{l|}{} \\
\hline Typical \# of failures & & & & & & \\
\hline Range & & & & & & \\
\hline
\end{tabular}

\begin{tabular}{|c|c|c|c|c|c|c|}
\hline Quality of SI & \multicolumn{6}{|c|}{ Poor (less than 98\%) } \\
\hline Vulnerability to Failure & \multicolumn{3}{|c|}{ Low } & \multicolumn{3}{|c|}{ Medium } \\
\hline Trade Volume (weekly) & $<40,000$ & 40,000 to 100,000 & $>100,000$ & $<40,000$ & 40,000 to 100,000 & $>100,000$ \\
\hline Typical \# of failures & & & & & & \\
\hline Range & & & & & & \\
\hline
\end{tabular}

\begin{tabular}{|l|c|l|l|l|l|l|}
\hline Quality of SI & \multicolumn{5}{|c|}{ Poor (less than 98\%) } \\
\hline Vulnerability to Failure & \multicolumn{5}{|c|}{ High } \\
\hline Trade Volume (weekly) & $<40,000$ & 40,000 to 100,000 & $>100,000$ \\
\hline Typical \# of failures & & & & & \\
\hline Range & & & & & & \\
\hline
\end{tabular}

Table A.2: Matrix format questionnaire - Number of settlement failure 
Appendix B

\section{Prior Probabilities}




\begin{tabular}{|l|l|l|}
\hline \multicolumn{3}{|c|}{ Quality of SI } \\
\hline SSI in place & Yes & No \\
\hline Excellent & 0.85 & 0.60 \\
Fair & 0.10 & 0.25 \\
Poor & 0.05 & 0.15 \\
\hline
\end{tabular}

\begin{tabular}{|l|r|}
\hline \multicolumn{2}{|c|}{$\begin{array}{c}\text { Backoffice-system- } \\
\text { failtime }\end{array}$} \\
\hline Not critical & 0.35 \\
Critical & 0.65 \\
\hline
\end{tabular}

\begin{tabular}{|l|r|r|r|r|}
\hline \multicolumn{4}{|c|}{ SSI in place } \\
\hline & \multicolumn{2}{|c|}{ Yes } & \multicolumn{2}{c|}{ No } \\
\hline Typical value & 0.95 & & 0.05 & \\
Range & 0.9 & 0.98 & 0.02 & 0.1 \\
\hline
\end{tabular}

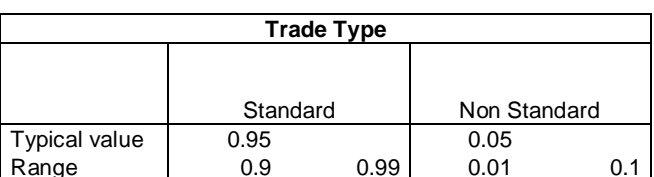

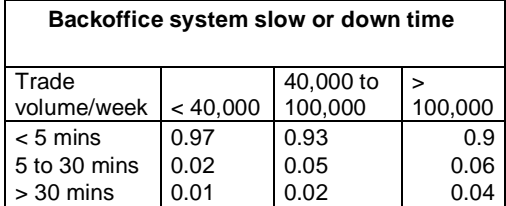

\begin{tabular}{|l|l|l|l|}
\hline \multicolumn{4}{|c|}{ Front office system } \\
\hline $\begin{array}{l}\text { Trade } \\
\text { volume/week }\end{array}$ & $<40,000$ & $\begin{array}{l}40,000 \\
100,000\end{array}$ & $>$ \\
\hline $\begin{array}{l}\text { Proper } \\
\text { function }\end{array}$ & 0.85 & 0.70 & 0.60 \\
Mal function & 0.10 & 0.20 & 0.25 \\
Not available & 0.05 & 0.10 & 0.15 \\
\hline
\end{tabular}

\begin{tabular}{|l|l|}
\hline \multicolumn{2}{|c|}{ Trade volume/week } \\
\hline less than & 0.75 \\
40,000 & 0.20 \\
40,000 to 100,000 & 0.05 \\
more than 100,000 & \\
\hline
\end{tabular}

\begin{tabular}{|l|l|l|}
\hline \multicolumn{3}{|c|}{ Trans_ipos } \\
\hline $\begin{array}{l}\text { Transmission } \\
\text { from }\end{array}$ & & \\
payment syst & STP & Manual \\
\hline Correct + timely & 0.99 & 0.80 \\
Incorrect + untimely & 0.01 & 0.20 \\
\hline
\end{tabular}

\begin{tabular}{|l|cc|cc|}
\hline \multicolumn{5}{|c|}{ Gross Netting } \\
\hline & Gross & \multicolumn{2}{|c|}{ Netting } \\
\hline Typical value & \multicolumn{2}{|c|}{0.9} & 0.1 & 0.2 \\
Range & 0.85 & 0.95 & 0.02 & 0.2 \\
\hline
\end{tabular}

\begin{tabular}{|l|lll|lll|}
\hline \multicolumn{9}{|c|}{ Payment system input method } \\
\hline $\begin{array}{l}\text { Backoffsys } \\
\text { failtime }\end{array}$ & \multicolumn{3}{c|}{ Critical } & & Not Critical \\
\hline Duration & $<5$ mins & 5 to 30 & $>30$ min & $<5$ mins & 5 to 30 & $>30$ min \\
\hline STP & 0.95 & 0.80 & 0.30 & 1.0 & 0.95 & 0.80 \\
Manual & 0.05 & 0.20 & 0.70 & 0.0 & 0.05 & 0.20 \\
\hline
\end{tabular}

\begin{tabular}{|c|c|c|c|c|c|c|}
\hline \multicolumn{7}{|c|}{ Vulnerability to failure } \\
\hline $\begin{array}{l}\text { Confirmation } \\
\text { matching }\end{array}$ & \multicolumn{2}{|c|}{ Done correctly } & \multicolumn{2}{|c|}{ Done incorrectly } & \multicolumn{2}{|c|}{$\begin{array}{c}\text { Not done (not sent, no } \\
\text { feedback, etc) }\end{array}$} \\
\hline Trade capture & $\begin{array}{l}\text { Correct } \\
\text { and } \\
\text { timely }\end{array}$ & Incorrect & $\begin{array}{l}\text { Correct } \\
\text { and } \\
\text { timely }\end{array}$ & Incorrect & $\begin{array}{l}\text { Correct } \\
\text { and } \\
\text { timely }\end{array}$ & Incorrect \\
\hline Low & 0.99 & 0.85 & 0.99 & 0.0 & 0.98 & 0.0 \\
\hline Medium & 0.01 & 0.10 & 0.01 & 0.10 & 0.01 & 0.15 \\
\hline High & 0.0 & 0.05 & 0.0 & 0.90 & 0.01 & 0.85 \\
\hline
\end{tabular}

\begin{tabular}{|c|c|c|c|c|c|c|c|c|}
\hline \multicolumn{9}{|c|}{ Confirmation Matching } \\
\hline SSI in place & \multicolumn{4}{|c|}{ Yes } & \multicolumn{4}{|c|}{ No } \\
\hline $\begin{array}{l}\text { Gross/Netting } \\
\text { settle }\end{array}$ & \multicolumn{2}{|c|}{ Gross settlement } & \multicolumn{2}{|c|}{ Netting settlement } & \multicolumn{2}{|c|}{ Gross settlement } & \multicolumn{2}{|c|}{ Netting settlement } \\
\hline Trade Type & Standard & $\begin{array}{l}\text { Non } \\
\text { Stand/Stru }\end{array}$ & Standard & $\begin{array}{l}\text { Non } \\
\text { Stand/Stru }\end{array}$ & Standard & $\begin{array}{l}\text { Non } \\
\text { Stand/Stru }\end{array}$ & Standard & $\begin{array}{l}\text { Non } \\
\text { Stand/Stru }\end{array}$ \\
\hline $\begin{array}{l}\text { Done correctly } \\
\text { Done } \\
\text { incorrectly } \\
\text { Not done }\end{array}$ & $\begin{array}{l}0.89 \\
0.01 \\
0.1\end{array}$ & $\begin{array}{l}0.85 \\
0.07 \\
0.08\end{array}$ & $\begin{array}{l}0.87 \\
0.05 \\
0.08\end{array}$ & $\begin{array}{l}0.80 \\
0.14 \\
0.06\end{array}$ & $\begin{array}{l}0.85 \\
0.07 \\
0.08\end{array}$ & $\begin{array}{l}0.82 \\
0.12 \\
0.06\end{array}$ & $\begin{array}{l}0.77 \\
0.18 \\
0.05\end{array}$ & $\begin{array}{l}0.75 \\
0.2 \\
0.05\end{array}$ \\
\hline
\end{tabular}

\begin{tabular}{|c|c|c|c|c|c|c|c|c|c|}
\hline \multicolumn{10}{|c|}{ Trade capture } \\
\hline $\begin{array}{l}\text { Trade } \\
\text { volume/day }\end{array}$ & $<8,00$ & & & 8,000 to 2( & 000 & & $>100$ & & \\
\hline $\begin{array}{l}\text { System } \\
\text { kondor }\end{array}$ & $\begin{array}{l}\text { prop } \\
\text { funct }\end{array}$ & mal funct & not avail & prop funct & mal funct & not avail & $\begin{array}{l}\text { prop } \\
\text { funct }\end{array}$ & mal funct & $\begin{array}{l}\text { not } \\
\text { avail }\end{array}$ \\
\hline $\begin{array}{l}\text { Correct+timely } \\
\text { Incorrect or } \\
\text { untime }\end{array}$ & $\begin{array}{l}0.98 \\
0.02\end{array}$ & $\begin{array}{l}0.90 \\
0.10\end{array}$ & $\begin{array}{l}0.70 \\
0.30\end{array}$ & $\begin{array}{l}0.95 \\
0.05\end{array}$ & $\begin{array}{l}0.85 \\
0.15\end{array}$ & $\begin{array}{l}0.60 \\
0.40\end{array}$ & $\begin{array}{l}0.90 \\
0.10\end{array}$ & $\begin{array}{l}0.80 \\
0.20\end{array}$ & $\begin{array}{l}0.55 \\
0.45\end{array}$ \\
\hline
\end{tabular}

Table B.1: Elicited probabilities - frequency of failure I 


\begin{tabular}{|c|c|c|c|c|c|c|c|c|c|c|c|}
\hline \multicolumn{2}{|c|}{ Node: "Fail Settlement" } & \multicolumn{10}{|c|}{ - Number of settlement failure } \\
\hline Quality of SI & \multicolumn{11}{|c|}{ Excellent (100\%) } \\
\hline Vulnerability to Failure & \multicolumn{5}{|c|}{ Low } & \multicolumn{6}{|c|}{ Medium } \\
\hline Trade Volume (weekly) & $\begin{array}{c}< \\
40,000\end{array}$ & & & & 000 & & 000 & & $\begin{array}{l}40,000- \\
100,000\end{array}$ & $>1$ & \\
\hline Typical \# of failures & 2 & & & & & & 2 & & 3 & & \\
\hline Range & \begin{tabular}{l|l}
0 & 2
\end{tabular} & 1 & 3 & 3 & 5 & 1 & 3 & 2 & 5 & 4 & 7 \\
\hline
\end{tabular}

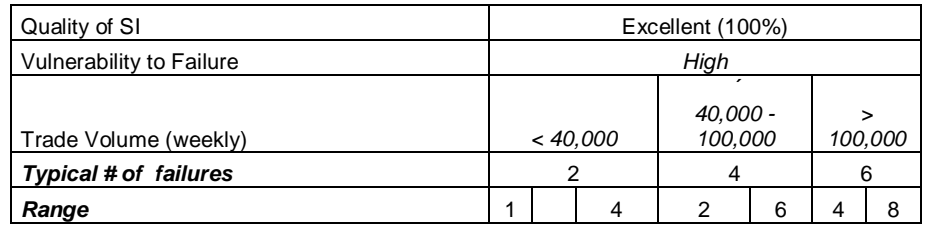

\begin{tabular}{|c|c|c|c|c|c|c|c|c|c|c|c|}
\hline Quality of SI & \multicolumn{11}{|c|}{ Fair (99- 100\%) } \\
\hline Vulnerability to Failure & \multicolumn{5}{|c|}{ Low } & \multicolumn{6}{|c|}{ Medium } \\
\hline Trade Volume (weekly) & 40,000 & & & & & 40 & & & $\begin{array}{l}40,000- \\
100,000\end{array}$ & \multicolumn{2}{|c|}{$>100,000$} \\
\hline Typical \# of failures & 2 & \multicolumn{2}{|c|}{4} & \multicolumn{2}{|c|}{6} & \multicolumn{2}{|c|}{3} & & 5 & \multicolumn{2}{|c|}{7} \\
\hline Range & \begin{tabular}{l|l}
1 & 4 \\
\end{tabular} & 2 & 6 & 4 & 8 & 1 & 5 & 3 & 7 & 5 & 10 \\
\hline
\end{tabular}

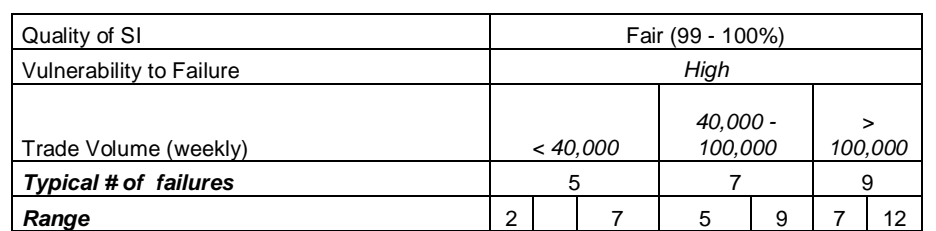

\begin{tabular}{|c|c|c|c|c|c|c|c|c|c|c|c|c|}
\hline Quality of SI & \multicolumn{12}{|c|}{ Poor (less than 99\%) } \\
\hline Vulnerability to Failure & \multicolumn{6}{|c|}{ Low } & \multicolumn{6}{|c|}{ Medium } \\
\hline Trade Volume (weekly) & & & & & & & 40 & & & $\begin{array}{l}0,000- \\
00,000\end{array}$ & \multicolumn{2}{|c|}{$>100,000$} \\
\hline Typical \# of failures & \multicolumn{2}{|c|}{5} & \multicolumn{2}{|c|}{7} & \multicolumn{2}{|c|}{9} & \multicolumn{2}{|c|}{5} & & 8 & \multicolumn{2}{|c|}{10} \\
\hline Range & 2 & 7 & 5 & 9 & 7 & 12 & 3 & 7 & 8 & 9 & 9 & 12 \\
\hline
\end{tabular}

\begin{tabular}{|c|c|c|c|c|}
\hline Quality of SI & \multicolumn{4}{|c|}{ Poor (less than 99\%) } \\
\hline Vulnerability to Failure & \multicolumn{4}{|c|}{ High } \\
\hline Trade Volume (weekly) & $<40,000$ & \multicolumn{2}{|c|}{$\begin{array}{l}40,000- \\
100,000\end{array}$} & 100,000 \\
\hline Typical \# of failures & 10 & \multicolumn{2}{|c|}{15} & 18 \\
\hline Range & 11 & 12 & 17 & 20 \\
\hline
\end{tabular}

Table B.2: Elicited probabilities - frequency of failure II 


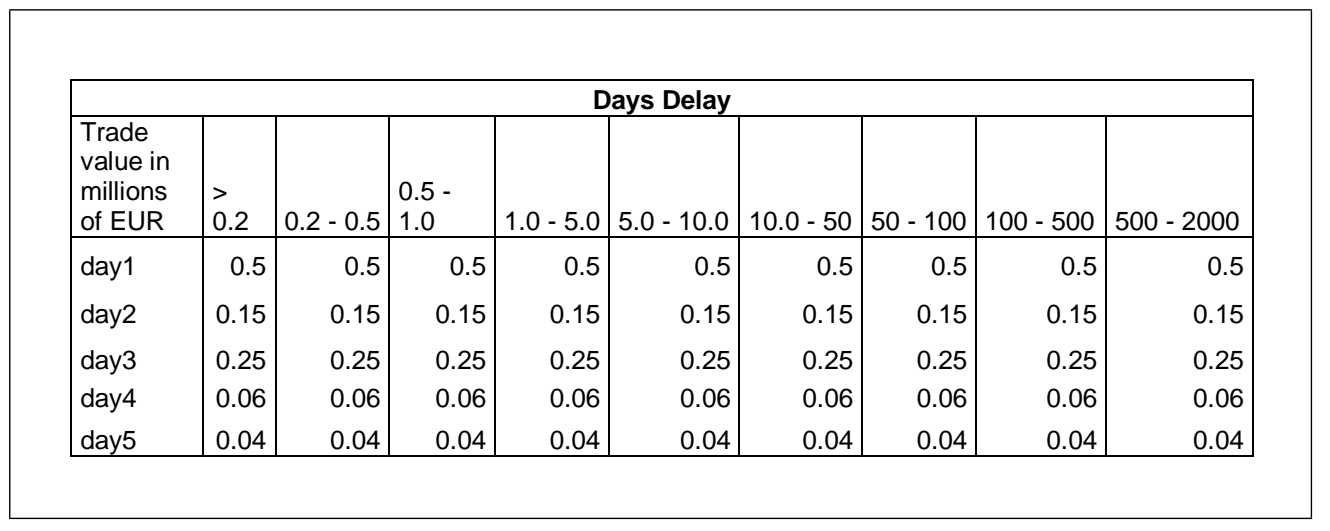

Table B.3: Elicited probabilities - loss severity network

\begin{tabular}{|l|r|r|r|r|r|r|r|r|r|}
\hline \multicolumn{7}{|c|}{ Days Delay (with logit adjustment for illustration S III) } \\
\hline $\begin{array}{l}\text { Trade } \\
\text { value in } \\
\text { millions of }\end{array}$ & & & & & & & & & \\
EUR & 0.2 & $0.2-0.5$ & $0.5-1.0$ & $1.0-5.0$ & $5.0-10.0$ & $10.0-50$ & $50-100$ & $100-500$ & $500-2000$ \\
\hline day1 & 0.5 & 0.5 & 0.5 & 0.5 & 0.5 & 0.5 & 0.83458 & 0.99029 & 0.99920 \\
day2 & 0.15 & 0.15 & 0.15 & 0.15 & 0.15 & 0.15 & 0.04823 & 0.00279 & 0.00023 \\
day3 & 0.25 & 0.25 & 0.25 & 0.25 & 0.25 & 0.25 & 0.08736 & 0.00526 & 0.00044 \\
day4 & 0.06 & 0.06 & 0.06 & 0.06 & 0.06 & 0.06 & 0.01800 & 0.00101 & 0.00008 \\
day5 & 0.04 & 0.04 & 0.04 & 0.04 & 0.04 & 0.04 & 0.01182 & 0.00066 & 0.00005 \\
\hline
\end{tabular}

Table B.4: Days delay node probabilities - with logit adjustments for illustration SIII in chapter 6 


\section{Bibliography}

C. Alexander. Operational Risk, regulation analysis and management. Pearson Education, London, 2003.

M. Alpert and H. Raiffa. A progress report on the training of probability assessors. In D. Kahnemann, P. Slovic, and A. Tverski, editors, Judgement under Uncertainty, Heuristics and Biases, pages 294-305. Cambridge University Press, Cambridge, 1982.

D.P. Ames, B.T. Neilson, D.K. Stevens, and U. Lall. Using Bayesian networks to model watershed management decisions: an East Canyon creek case study. World Wide Web, http://www.hydromap.com/papers/ames_ecc.pdf, 2003. Visited July, 2005.

Bank for International Settlement. Triennial Central Bank Survey: foreign exchange and derivatives market activity in 2001. Bank for International Settlements, Basel, 2002.

Bank for International Settlement. Triennial Central Bank Survey: foreign exchange and derivatives market activity in 2004. Bank for International Settlements, Basel, 2005.

Basel Committee on Banking Supervision. Operational Risk, Consultative paper 2. Bank for International Settlements, Basel, 2001.

Basel Committee on Banking Supervision. Sound Practices for the management and supervision of Operational risk. Bank for International Settlements, Basel, 2002 .

Basel Committee on Banking Supervision. Operational risk - Rules language (draft). Bank for International Settlements, Basel, 2003.

T. Blunden. Scorecard approaches. In C. Alexander, editor, Operational risk, regulation, analysis and management, London, 2003. Pearson Education. 
J.H. Boose and M. Shaw. Knowledge acquisition for knowledge based systems. In Eleventh International Conference on Artificial Intelligence, Detroit, Mechigan, 1989 .

R.A. Brauldi. Introductory Combinatories. Elsevier Science Publishing Co.,Inc., New York, 1992.

J.S. Breese and D. Heckerman. Decision theoretical troubleshooting. IEEE Transactions on Systems, Man and Cybernetics, Part A (Systems and Human), 26 (6):838-842, 1999.

M.J. Brewer. A model-based approach for variable bandwidth selection in kernel density estimation. Journal of Statistics and Computing, 10:229-310, 2000.

M.G. Bulmer. Principles of Statistics. Dover, New York, 1979.

R. Carnap. Logical Foundations of Probabilities. Routledge and Kegan Paul, 1950.

E. Charniak. Bayesian networks without tears. Artificial Intelligence Magazine, 12(4):50-60, 1991.

J. Cheng and M.J. Druzdzel. Ais-bn: An adaptive importance sampling algorithm for evidential reasoning in large bayesian networks. Journal of Artificial Intelligence Research, 13:155-188, 2000.

D.A Cleaves. Cognitive biases and corrective techniques: Proposals for improving elicitation procedures for knowledge-based systems. In Proceedings from the AAAI sponsored 2nd Annual Knowledge Acquisition for Knowledge-Based Systems Workshop, pages 9-0-9-11, 1986.

R.M. Cooke and L.H. Goossens. Procedures guide for structured expert judgement in accident consequence modelling. Radiation Protection Dosimetry, 90 (3):303-309, 2000.

G.F. Cooper. The computational complexity of probabilistic inference using bayesian belief networks. Artificial Intelligence, 42:393-405, 1990.

R. Cowell. Introduction to inference for bayesian networks. In Jordan, pages 9-26, 1999.

R.G. Cowell and A.P. Dawid. Fast retraction of evidence in a probabilistic expert system. Statistics and Computing, 2:37-40, 1992. 
M.G. Cruz. Modeling, measuring and hedging operational risk. John Wiley and Sons, West Sussex, England, 2002.

P. Dagum and M. Luby. Approximating probabilistic inference in bayesian belief networks is np-hard. Artificial Intelligence, 60:141-153, 1993.

A. Darwiche. A differential approach to inference in bayesian networks. In Uncertainty in Artificial Intelligence 2000, 2000.

B. de Fenetti. The true SP problem (subjective probability problem) versus various more or less related side problems. In Proceedings of a Research Conference on Subjective Probability and Related Fields, Psychological Institute of the University of Hamburg, pages 33-42, 1969.

European Banking Federation. European interbank compensation guidelines. World Wide Web, http://www.bba.org.uk/content/1/c4/35/56/45900. pdf, 2001. Visited July, 2005.

N.E. Fenton, P. Krause, and M. Neil. A probabilistic model for software defect prediction. IEEE Trans Software Engineering, 2001.

Foreign Exchange Committe. Management of Operational Risk in Foreign Exchange. Federal Reserve Bank of New York, New York, 2003.

Foreign Exchange Committee. Guidelines for the management of FX trading activities. In The Foreign Exchange Committee 2000 Annual Report, pages 69-70, New York, 2001. Federal Reserve Bank of New York.

R. Fung and K.C. Chang. Weighting and integrating evidence for stochastic simulation in bayesian networks. Uncertainty in Artificial Intelligence, 5:209219, 1989.

R. Fung and B.D Favero. Backward simulation in bayesian networks. In R. Lopez de Mantaras and D. Poole, editors, Proceedings of the Tenth Conference on Uncertainty in Artificial Intelligence, pages 227-234, 1994.

J.L. Galan Caballero. Measuring the effect of organisational factors using Bayesian Networks. PhD thesis, Queen Mary University, part of the University of London, 2005. draft.

P. Giudici. Integrating quantitative and qualitative operational risk data. In M. Cruz, editor, Operational risk modeling and Analysis. Risk Books, London, 2004. 
Group of Thirty. Clearance and Settlement Systems in the World's Securities Markets. Group of Thirty, Washington, D.C., 1989.

H. Guo and W. Hsu. A survey on algorithms for real-time bayesian network inference. In the joint AASI-02/KDD-02/UAI-02 workshop on Real-Time Decision Support and Diagnosis Systems, 2002.

O. Hansson and A. Mayer. Heuristic search as evidential reasoning. In Proceedings of the 5th Workshop on Uncertainty in Artificial Intelligence, 1989.

M. Haubenstock. The operational risk management framework. In C. Alexander, editor, Operational risk, regulation, analysis and management, London, 2003. Pearson Education.

D. Heckermann. Bayesian networks for data mining. Data mining and knowledge discovery, 1:79-119, 1997.

D. Heckermann and J.S. Breese. Causal independence for probability assessment and inference using bayesian networks. IEEE Transactions on Systems, Man and Cybernetics, 26:826-831, 1996.

M. Henrion. Some practical issues in constructing belief networks. In Uncertainty in Artificial Intelligence 3. 1989.

Industry Technical Working Group. An LDA based AMA for the measurement of operational risk- ideas issues and emerging practice. World Wide Web, address $=$ http://www.gloriamundi.org/picsresources/con0529p \_1.pdf, 2003. Visited July, 2005.

F.V. Jensen. Bayesian networks and decision graphs. Springer, New York, 2001.

F.V. Jensen, C. Skaanning, and U. Kjaerulff. The sacso system for troubleshooting of printing systems. Technical report, University of Aalborg, Denmark, 2000 .

J.P. Morgan and Reuters. Riskmetrics - Technical Document. World Wide Web, http://www.riskmetrics.com/rmcovv.html, 1996. Visited July, 2005.

J.B Kandane and L.J. Wofson. Experiences in elicitation. The Statistician, 47 (1):3-9, 1998 .

J.L. King. Operational Risk: measurement and modeling. John Wiley and Sons, West Sussex, England, 2001. 
U. Kjaerulff. Reduction of computational complexity in bayesian networks through removal of weak dependencies. In Proceedings of the Tenth Conference on Uncertainty in Artificial Intelligence, 1994.

A.H. Lau and T.Y. Leong. Probes: A framework for probability elicitation from experts. pages 301-305, 1999.

S.L. Lauritzen and D.J. Spiegelhalter. Local computations with probabilities on graphical structures and their applications to expert systems. In Proceedings of the Royal Statistical Society, volume 50, pages 154-227, 1988.

S.L. Lauritzen, A.P. Dawid, B.N Larsen, and H.G. Leimer. Independence properties of directed markov fields. Networks, 20:491-505, 1990.

Z. Li and B. D'Ambrosio. Efficient inference in bayes networks as a combinatorial optimization problem. International Journal of Approximate Reasoning, 11:5581, 1994.

S. Lichtenstein, P. Slovic, B. Fischhoff, M. Layman, and B. Combs. Judge frequency of lethal events. Experimental Psychology: Human Learning and Memory, 4(6):551-578, 1978.

S. Lichtenstein, B. Fischhoff, and L. Philips. Calibration of probabilities: The state of the art to 1980. In Judgement under uncertainty: Heuristics and biases. Cambridge University Press, Cambridge, 1982.

D. V. Lindley, A. Tversky, and R.V. Brown. On the reconciliation of probability assessments (with discussions). Royal Statistics Society, A, 142:146-180, 1979.

C. Loader. Local Regression and Likelihood. Springer, New York, 1999.

W. Long. Medical diagnosis using a probabilistic causal network. Applied Artificial Intelligence, 3:367-383, 1989.

E. Ludwig. The Ludwig Report. World Wide Web, http://www. allfirst.com/ Ludwigreport.pdf, 2002. Visited March, 2002.

B.G. Marcot, R.S. Holthausen, M.G. Raphael, M. Rowland, and M. Wisdom. Using bayesian belief networks to evaluate fish and wildlife population viability under land management alternatives from an environmental impact statement. Forest Ecology and Management, 153:29-42, 2001. 
M. A. Meyer and J.M. Booker. Eliciting and Analyzing Expert Judgement: A Practical Guide. American Statistical Society and the Society for Industrial and Applied Mathematics, United States of America, 2001.

M.G. Morgan and M. Henrion. Uncertainty: A guide to Dealing with Uncertainty in Quantitative Risk and Policy Analysis. Cambridge University Press, Cambridge, 1990.

M. Neil, N.E. Fenton, and M. Tailor. Using bayesian networks to model expected and unexpected operational losses. Risk Analysis, 2005. Accepted for publication.

D. Nikovski. Constructing bayesian networks for medical diagnosis from incomplete and partially correct statistics. IEEE Transactions on Knowledge and Data Engineering, 12(4):509-516, 2000.

K.G. Olesen, U. Kjaerulff, F.V. Jensen, F. Jensen, B. Falck, S. Andreassen, and S.K. Andersen. A munin network for the median nerve - a case study on loops. Applied Artificial Intelligence, 3:385-404, 1989.

K.G. Olesen, S.L. Lauritzen, and F.V. Jensen. aHugin: A system creating adaptive causal probabilistic networks. In The Eighth Conference on Uncertainty in Artificial Intelligence, pages 223-229, 1992.

J. Pearl. Causality: Models, Reasoning and Inference. Cambridge University Press, Cambridge, 2000.

J. Pearl. Probabilistic reasoning in Intelligence Systems: Networks of Plausible Inference. Morgan Kaufman Publishers, San Mateo, CA, 1988.

J. Pearl and S. Russel. Bayesian networks. In M. Arbib, editor, Handbook of Brian Theory and Neural Networks. MIT Press, 2001.

J. Perera. Operational risk models - Neural networks do all the work. Operational Risk - Supplement to Risk Magazine, 2000.

P.C. Price. Effects of a relative-frequency elicitation question on likelihood judgement accuracy: The case of external correspondence. Organisational Behaviour and Human Decision Processes, 76(3):277-297, 1998.

S. Ramamurthy, H. Arora, and A. Ghosh. Operational risk and probabilistic networks - an application to corporate actions processing. World Wide Web, address $=$ http://www.hugin.com/cases/Finance/Infosys/oprisk.article, 2005. Visited July, 2005. 
Scenario Based AMA Working Group. Scenario based AMA. World Wide Web, address = http://www.gloriamundi.org/picsresources/con0529d $\backslash$ _1.pdf, 2003. Visited July, 2005.

R. Shacter. Intelligent probabilistic inference. Uncertainty in Artificial Intelligence, pages 371-382, 1986.

R. Shacter. Evidence absorption and propagation through evidence reversals. Uncertainty in Artificial Intelligence, 1990.

S.D. Shacter and M. Peot. Simulation approaches to general probabilistic inference on belief networks. In Proceedings of the Conference in Artificial Intelligence, 1990.

S.D. Shacter, B. D'Ambrosio, and B.D. Del Favero. Symbolic probabilistic inference in belief networks. In Proceedings of the Eighth National Conference on Artificial Intelligence, 1990.

J.A. Spencer. A further study of estimating averages. Economics, 6:255-265, 1963.

D.J. Spiegelhalter and S.L. Lauritzen. Sequential updating of conditional probabilities on directed graphical structures. Networks, 20:579-605, 1990.

T.A. Stephenson. An introduction to bayesian network theory and usage. Research report, idiap-rr 00-03, Dalle Molle Institute for Perceptual Artificial Intelligence (IDIAP), 2000.

R.G Swanson. Intuitive estimates of means. Psychonomic Science, 5(4):161-162, 1966.

A. Tversky and D. Kahneman. Judgement under uncertainty: Heuristics and biases. Management Science, 185:1124-1131, 1974.

G.J. van den Brink. Operational Risk, new challenges for banks. Palgrave, New York, 2002.

L.C. van der Gaag, S. Renooij, C.L.M Witteman, B.M.P Aleman, and B.G. Tall. How to elicit many probabilities. In Proceedings of the Fifteenth Annual Conference on Uncertainty in Artificial Intelligence, 1999.

L.C. van der Gaag, S. Renooij, and C.L. Witteman. Probabilities for a probabilistic network: a case study in oesophageal cancer. Artificial Intelligence Med, 25 (2):123-148, 2002. 
R.A van Engelen. Approximating bayesian networks by arc removal. IEEE Transactions on Pattern Analysis and Machine Intelligence, 19:916-920, 1997.

D. von Winterfeldt and W. Edwards. Decision Analysis and Behavioural Research. Cambridge University Press, Cambridge, 1986.

T.S. Wallsten and D.V. Budescu. Encoding subjective probabilities: A psychological and psychometric review. Management Science, 29:151-173, 1983.

R.L. Winkler. The assessment of prior distributions in bayesian analysis. Journal of American Statistical Association, 1967.

N.L. Zhang and D. Poole. A simple approach to bayesian network computations. In Proceedings of the Tenth Canadian Conference on Artificial Intelligence, page 171, 1994. 


\section{Curriculum Vitae \\ Personal Data}

Name:

Address:

Date/place of birth:

Nationality:

Educational Record

Oct.2002-Sept.2005:

Sept.2000-May 2002:

Sept. 1998-2000:

Oct. 1991-1996:

Sept.1988-June 1990

Sept 1983-June 1988

\section{Experience Record}

Oct.2001-April 02

$1996-1998$

\author{
Kwabena ADUSEI-POKU \\ Albrecht-Thaer-Weg 22A/01 \\ 37075 Goettingen \\ 17.11.1969, Kumasi, Ghana \\ Ghanaian
}

Ph.D at the Institute of Statistics and Econometrics Faculty of Economics and Business Administration of the University of Goettingen.

MBA-International Management, Stuttgart Institute of Management and Technology, Stuttgart, Germany

M.Sc. Water Resources Engineering and Management University of Stuttgart, Germany

B.Sc. (Honours) Civil Engineering, University of

Science and Technology, Kumasi, Ghana

G.C.E Advanced level, Presby Boys Secondary

School, Accra, Ghana

G.C.E Ordinary Level, Accra Academy, Accra, Ghana
Student Project with Deutsche Bank

International Risk Management - Assessment and Analysis of Risk Management systems of some selected Medium Sized Enterprises in South West Germany for Deutsche Bank South West.

Student Worker

HypoVereins Bank-Global Project Finance

(Water, Regenerative Energy, Transportation

and PFI Teams), Munich, Germany

Water Supply Engineer, Colan Consult and

Burgeap consulting, Accra Ghana.

Civil and Site Engineer, Colan Consult. 
